\title{
One-pot Assembly of 3-Hydroxycarbazoles via Uninterrupted Propargylation/Hydroxylative Benzannulation Reactions
}

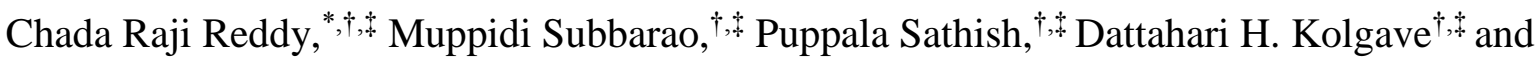 \\ Ramachandra Reddy Donthiri ${ }^{\dagger}$ \\ †Department of Organic Synthesis \& Process Chemistry, CSIR-Indian Institute of Chemical \\ Technology, Hyderabad 500007, India. ${ }^{\star}$ Academy of Scientific and Innovative Research \\ (AcSIR), Ghaziabad 201 002, India.E-mail: rajireddy@iict.res.in
}

\section{Table of Contents}

1. General information $\mathrm{S} 1-\mathrm{S} 2$

2. Experimental procedures and characterization data of compounds S2-S24

3. Gram scale reaction procedure for $\mathbf{3 a}$

4. X-ray analysis data of $\mathbf{3 a}$

5. References

6. ${ }^{1} \mathrm{H}$ NMR and ${ }^{13} \mathrm{C}$ NMR spectral copies of compounds

7. HRMS spectral copy of $\mathrm{H}_{2} \mathrm{O}^{18}$ experiment.

\section{General information:}

All the reactions were performed in oven-dried glass apparatus, the air and moisture sensitive reactions were carried out under inert atmosphere (nitrogen) using freshly distilled anhydrous solvents. Commercially available reagents were used as such without further purification. All reactions were monitored by thin-layer chromatography carried out on silica plates using UVlight and anisaldehyde for visualization. Column chromatography was performed on silica gel (100-200 mesh) using hexanes and ethyl acetate as eluent. ${ }^{1} \mathrm{H}$ NMR was recorded in $\mathrm{CDCl}_{3}$ on $500 \mathrm{MHz}, 400 \mathrm{MHz}$ and $300 \mathrm{MHz}$ and ${ }^{13} \mathrm{C} \mathrm{NMR}$ was recorded on $125 \mathrm{MHz}, 100 \mathrm{MHz}$ and $75 \mathrm{MHz}$. Chemical shifts were reported in $\delta(\mathrm{ppm})$ relative to TMS as an internal standard and $J$ values were given in $\mathrm{Hz}$ (hertz). Multiplicity is indicated as, s (singlet); d (doublet); $\mathrm{t}$ (triplet); $\mathrm{m}$ (multiplet); dd (doublet of doublets), etc. $\delta 7.26$ and $\delta 1.56$ are corresponding to $\mathrm{CDCl}_{3}$ and moisture respectively in ${ }^{1} \mathrm{H} \mathrm{NMR}, \delta 77.77$ is related to $\mathrm{CDCl}_{3}$ in ${ }^{13}$ C NMR. FT-IR spectra were recorded on Alpha (Bruker) Infrared Spectrophotometer. High resolution mass spectra (HRMS) [ESI+] were obtain using either a TOF or a double focusing spectrometer. 
All the Propargylic alcohols $2 \mathbf{a}$ to $2 \mathbf{o}$ and $\mathbf{6 a}$ and $\mathbf{6 b}$ were prepared based on literature reports $(2 \mathrm{a}, 2 \mathrm{~b}, 2 \mathrm{c}, 2 \mathrm{~d}, 2 \mathrm{e}, 2 \mathrm{f}, 2 \mathrm{~h}$ and $2 \mathrm{i}),{ }^{1 \mathrm{a}} 2 \mathrm{~g},{ }^{1 \mathrm{~b}} 2 \mathrm{~K},{ }^{1 \mathrm{c}} 2 \mathrm{j},{ }^{1 \mathrm{~d}}(2 \mathrm{l}, 2 \mathrm{~m}$ abd $2 \mathrm{n}),{ }^{1 \mathrm{e}} 2 \mathrm{o}^{1 \mathrm{f}} 6 \mathrm{a}^{1 \mathrm{~g}} 6 \mathrm{bb} .^{\mathrm{h}}$<smiles>OC(C#Cc1ccccc1)c1ccccc1</smiles>

$2 a$<smiles>OC(C#Cc1ccccc1)c1ccc(Br)cc1</smiles>

2d<smiles>OC(C#Cc1ccccc1)c1cccc2ccccc12</smiles>

$2 \mathrm{~g}$<smiles>OC(C#Cc1ccc(C(F)(F)F)cc1)c1ccccc1</smiles><smiles>C#CC(O)c1ccc(OC)cc1</smiles>

$2 m$<smiles>COc1ccc(C(O)C#CC#Cc2ccccc2)cc1</smiles><smiles>Cc1ccc(C(O)C#Cc2ccccc2)cc1</smiles>

2b<smiles>O=[N+]([O-])c1ccc(C(O)C#Cc2ccccc2)cc1</smiles>

$2 e$<smiles>Cc1ccc(C#CC(O)c2ccccc2)cc1</smiles>

$2 h$<smiles>CCCC#CC(O)c1ccccc1</smiles>

2k<smiles>C#CC(O)c1cccc2ccccc12</smiles>

$2 n$<smiles>COc1ccc(C(O)C#Cc2ccccc2)cc1</smiles><smiles>N#Cc1ccc(C(O)C#Cc2ccccc2)cc1</smiles>

$2 f$<smiles>COc1ccc(C#CC(O)c2ccccc2)cc1</smiles>

2i<smiles>C#CC(O)c1ccccc1</smiles>

2I<smiles>COc1cc(C(O)C#Cc2ccccc2)cc(OC)c1OC</smiles>

2. Experimental procedures and characterization data of compounds:

A. General Procedure for the Synthesis of indoloyl-2-ketones (1c-j): 


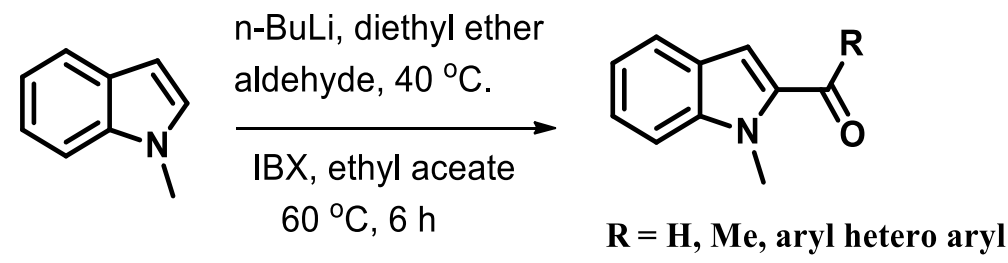

$2.5 \mathrm{M}$ n-BuLi ( 2 equiv) in hexane was added to a solution of $\mathrm{N}$-methylindole (1 equiv) in dry diethyl ether $(20 \mathrm{~mL})$ at $0{ }^{\circ} \mathrm{C}$ under nitrogen atmosphere. The mixture was stirred for $2 \mathrm{~h}$ at $40{ }^{\circ} \mathrm{C}$ before aldehyde (1.1 equiv) was added. The reaction mixture was allowed to stir for $4 \mathrm{~h}$ at room temperature. The aqueous solution was extracted with EtOAc $(3 \mathrm{X} 50 \mathrm{~mL})$ after quenching with a saturated aqueous $\mathrm{NH}_{4} \mathrm{Cl}$ solution. The combined organic layers were washed with brine solution $(50 \mathrm{~mL})$. After the organic layer was dried with $\mathrm{Na}_{2} \mathrm{SO}_{4}$, the solvent was removed under reduced pressure to get crude $2^{\circ}$-alcohol, which was directly subjected for oxidation. To the crude alcohol in EtOAc $(10 \mathrm{~mL}), \mathrm{IBX}(1.5$ equiv) was added and stirred for $2 \mathrm{~h}$ at $60{ }^{\circ} \mathrm{C}$. After that the reaction mixture was filtered on celite pad and washed with ethyl acetate $(2 \times 20 \mathrm{~mL})$. The filtrate was concentrated under reduced pressure, obtained concentrated reaction mixture was purified by column chromatography on silica gel (100- 200) using petroleum ether and EtOAc as eluent to obtain indoloyl-2-ketones (1cj).

\section{(1-Methyl-1H-indol-2-yl)(phenyl)methanone (1c):}

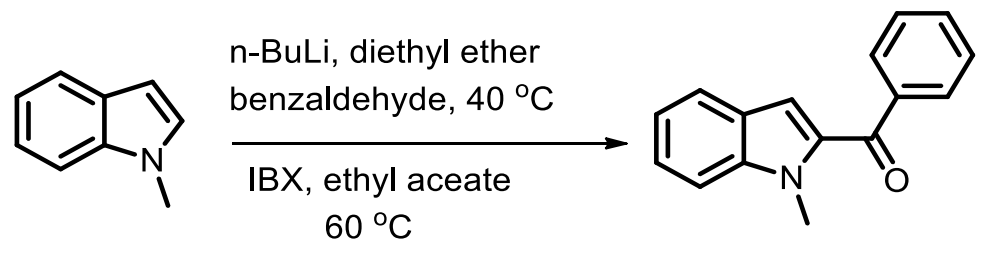

Following the general procedure A, $N$-methylindole $(1 \mathrm{~g}, 7.63 \mathrm{mmol})$ in diethyl ether was allowed to react with benzaldehyde $(891 \mathrm{mg}, 8.39 \mathrm{mmol})$ in the presence of $\mathrm{n}-\mathrm{BuLi}(6.1 \mathrm{ml}, 2.5 \mathrm{M})$ to get crude 1-methyl-1H-indol-2-yl)(phenyl)methanol, which was further oxidized with IBX (3.2 g, $11.42 \mathrm{mmol}$ ) to obtain (1-methyl-1H-indol-2-yl)(phenyl)methanone (1c), $1.5 \mathrm{~g}, 83 \%$ yield, brown oil, $R_{\mathrm{f}}=0.5$ (petroleum ether: EtOAc $\left.=8: 2\right) ;{ }^{1} \mathrm{H}$ NMR $\left(300 \mathrm{MHz}, \mathrm{CDCl}_{3}\right) \delta 7.92(\mathrm{~d}, J=7.2$ $\mathrm{Hz}, 2 \mathrm{H}), 7.67(\mathrm{~d}, J=8.0 \mathrm{~Hz}, 1 \mathrm{H}), 7.60(\mathrm{t}, J=7.3 \mathrm{~Hz}, 1 \mathrm{H}), 7.55-7.37(\mathrm{~m}, 4 \mathrm{H}), 7.23-7.12(\mathrm{~m}$, 1H), 7.02 (s, 1H), 4.13 (s, 3H); $\left.{ }^{13} \mathrm{C} \mathrm{NMR} \mathrm{(101} \mathrm{MHz,} \mathrm{CDCl}_{3}\right) \delta 188.7,140.3,139.5,135.0$, 132.2, 129.8, 128.3, 126.0, 125.9, 123.0, 120.8, 114.9, 110.4, 32.0; IR (neat): 3057, 2938, 1633, 1513, 1261, 942, $732 \mathrm{~cm}^{-1}$; HRMS (ESI): $\mathrm{m} / z$ calcd for $\mathrm{C}_{16} \mathrm{H}_{14} \mathrm{NO}(\mathrm{M}+\mathrm{H})^{+}: 236.1075$, found: 236.1071 . 


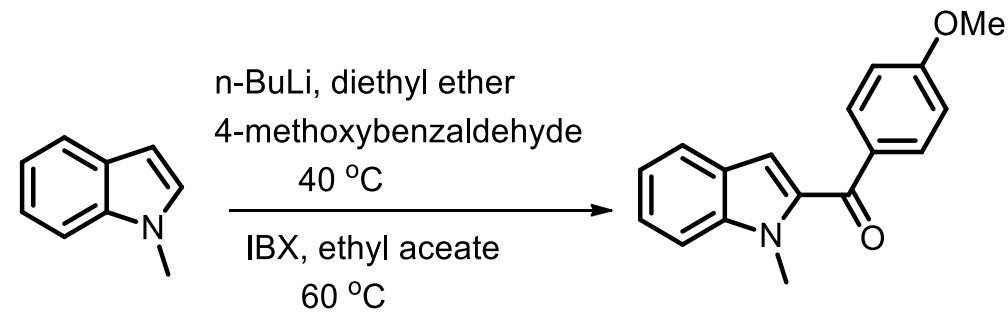

Following the general procedure A, $N$-methylindole $(1 \mathrm{~g}, 7.63 \mathrm{mmol})$ in diethyl ether was allowed to react with 4-methoxybenzaldehyde $(1.1 \mathrm{~g}, 8.39 \mathrm{mmol})$ in the presence of n-BuLi $(6.1 \mathrm{~mL}$, $2.5 \mathrm{M})$ to get crude (4-methoxyphenyl)(1-methyl- $1 H$-indol-2-yl)methanol, which was further oxidized with IBX (3.2 g, $11.42 \mathrm{mmol})$ to obtain (4-methoxyphenyl)(1-methyl-1H-indol-2yl)methanone (1d), $1.6 \mathrm{~g}, 80 \%$ yield, off-white solid, $R_{\mathrm{f}}=0.5$ (petroleum ether: EtOAc $=8: 2$ ); mp: 84-86 ${ }^{\circ} \mathrm{C}$; ${ }^{1} \mathrm{H}$ NMR $\left(400 \mathrm{MHz}, \mathrm{CDCl}_{3}\right) \delta$ 8.00-7.92 (m, 2H), 7.71-7.64 (m, 1H), 7.46$7.35(\mathrm{~m}, 2 \mathrm{H}), 7.20-7.13(\mathrm{~m}, 1 \mathrm{H}), 7.01-6.93(\mathrm{~m}, 3 \mathrm{H}), 4.07$ (s, 3H), $3.89(\mathrm{~s}, 3 \mathrm{H}) ;{ }^{13} \mathrm{C}$ NMR $\left(101 \mathrm{MHz} \mathrm{CDCl}_{3}\right) \delta 187.3,163.2,140.1,135.4,132.2,132.0,126.0,125.6,122.9,120.7$, 113.6, 113.5, 110.3, 55.6, 31.9; IR (KBr): 3054, 2936, 1623, 1413, 1161, 932, $732 \mathrm{~cm}^{-1}$; HRMS (ESI): $m / z$ calcd for $\mathrm{C}_{17} \mathrm{H}_{16} \mathrm{NO}_{2}(\mathrm{M}+\mathrm{H})^{+}: 266.1181$, found: 266.1183 .

(1-Methyl-1H-indol-2-yl)(4-(trifluoromethyl)phenyl)methanone (1e):

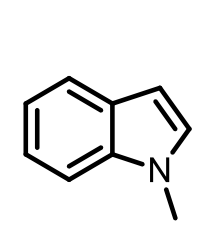

n-BuLi, diethyl ether 4-(trifluoromethyl)benzaldehyde $40^{\circ} \mathrm{C}$

IBX, ethyl aceate $60{ }^{\circ} \mathrm{C}$

Following the general procedure A, $N$-methylindole $(1 \mathrm{~g}, 7.63 \mathrm{mmol})$ in diethyl ether was allowed to react with 4-(trifluoromethyl)benzaldehyde $(1.6 \mathrm{~g}, 8.39 \mathrm{mmol})$ in the presence of $\mathrm{n}$-BuLi $(6.1$ $\mathrm{mL}, 2.5 \mathrm{M})$ to get crude (1-methyl-1H-indol-2-yl)(4-(trifluoromethyl)phenyl)methanol, which was further oxidized with IBX (3.2 g, $11.42 \mathrm{mmol})$ to obtain (1-methyl-1H-indol-2-yl)(4(trifluoromethyl)phenyl)methanone (1e), $1.8 \mathrm{~g}, 77 \%$ yield, off-white solid, $R_{\mathrm{f}}=0.5$ (petroleum ether: EtOAc = 8:2); mp: 122-124 ${ }^{\circ} \mathrm{C} ;{ }^{1} \mathrm{H}$ NMR $\left(400 \mathrm{MHz}, \mathrm{CDCl}_{3}\right) \delta 8.00(\mathrm{~d}, J=8.0 \mathrm{~Hz}, 2 \mathrm{H})$, $7.77(\mathrm{~d}, J=8.1 \mathrm{~Hz}, 2 \mathrm{H}), 7.72-7.65(\mathrm{~m}, 1 \mathrm{H}), 7.48-7.40(\mathrm{~m}, 2 \mathrm{H}), 7.22-7.15(\mathrm{~m}, 1 \mathrm{H}), 6.99$ (s, $1 \mathrm{H}), 4.15(\mathrm{~s}, 3 \mathrm{H}) ;{ }^{13} \mathrm{C}$ NMR $\left(126 \mathrm{MHz}, \mathrm{CDCl}_{3}\right) \delta 187.4,142.6,140.6,134.4,133.5$ (q, $J=$ $32.7 \mathrm{~Hz}), 129.8,126.6,125.8,125.2,123.8(\mathrm{q}, J=272.6 \mathrm{~Hz}), 123.2,121.1,115.7,110.5$, 
104.0, 32.1; IR (KBr): 3057, 2947, 2618, 1645, 1328, 1131, $757 \mathrm{~cm}^{-1}$; HRMS (ESI): $\mathrm{m} / z$ calcd for $\mathrm{C}_{17} \mathrm{H}_{13} \mathrm{NOF}_{3}(\mathrm{M}+\mathrm{H})^{+}$: 304.0949, found: 304.0944 .

(1-Methyl-1H-indol-2-yl)(2-methylthiophen-3-yl)methanone(1f):

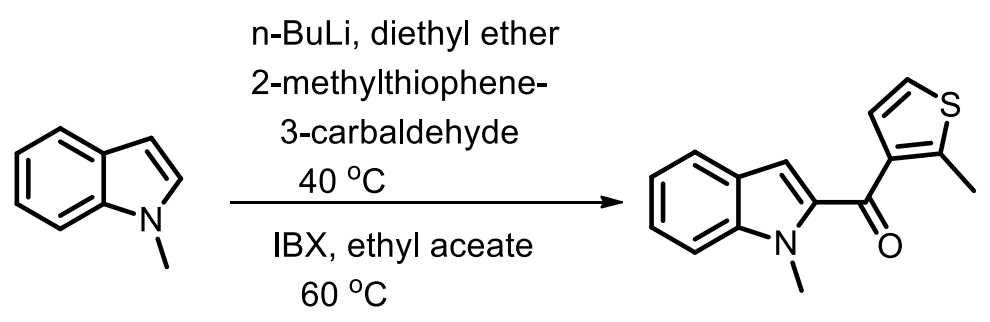

Following the general procedure A, $N$-methylindole $(1 \mathrm{~g}, 7.63 \mathrm{mmol})$ in diethyl ether was allowed to react with 2-methylthiophene-3-carbaldehyde $(1 \mathrm{~g}, 8.39 \mathrm{mmol})$ in the presence of $\mathrm{n}$-BuLi $(6.1$ $\mathrm{mL}, 2.5 \mathrm{M})$ to get crude (1-methyl-1H-indol-2-yl)(2-methylthiophen-3-yl)methanol, which was further oxidized with IBX (3.2 g, $11.42 \mathrm{mmol})$ to obtain (1-methyl-1H-indol-2-yl)(2methylthiophen-3-yl)methanone(1f), $1.4 \mathrm{~g}, 72 \%$ yield, yellow oil, $R_{\mathrm{f}}=0.5$ (petroleum ether: EtOAc = 8:2); ${ }^{1} \mathrm{H}$ NMR $\left(400 \mathrm{MHz}, \mathrm{CDCl}_{3}\right) \delta$ 7.73-7.66 (m, 1H), $7.48(\mathrm{~d}, J=5.0 \mathrm{~Hz}, 1 \mathrm{H})$, 7.45-7.36 (m, 2H), 7.26-7.23 (m, 1H), 7.20-7.13 (m, 1H), 7.03 (d, J = 5.0 Hz, 1H), 4.05 (s, 3H), $2.60(\mathrm{~s}, 3 \mathrm{H}) ;{ }^{13} \mathrm{C} \mathrm{NMR}\left(101 \mathrm{MHz}, \mathrm{CDCl}_{3}\right) \delta 180.9,145.7,140.1,136.6,135.1,132.1$, 130.3, 125.8, 125.7, 123.0, 120.7, 113.2, 110.3, 31.8, 16.6; IR (neat): 3047, 2917, 2658, 1745, 1228, 1126, $737 \mathrm{~cm}^{-1}$; HRMS (ESI): $\mathrm{m} / z$ calcd for $\mathrm{C}_{15} \mathrm{H}_{14} \mathrm{NOS}(\mathrm{M}+\mathrm{H})^{+}$: 256.0796, found: 256.0798 .

\section{1-(1-Methyl-1H-indol-2-yl)ethan-1-one(1g):}

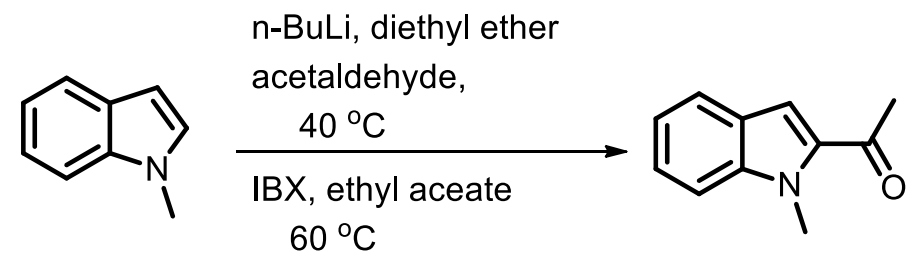

Following the general procedure $\mathrm{A}, \mathrm{N}$-methylindole $(1 \mathrm{~g}, 7.63 \mathrm{mmol})$ in diethyl ether was allowed to react with acetaldehyde $(2.1 \mathrm{~mL}, 38.15 \mathrm{mmol})$ in the presence of $\mathrm{n}-\mathrm{BuLi}(6.1 \mathrm{ml}, 2.5 \mathrm{M})$ to get crude 1-(1-methyl-1H-indol-2-yl)ethan-1-ol, which was further oxidized with IBX (3.2 g, 11.42 mmol) to obtain 1-(1-methyl-1H-indol-2-yl)ethan-1-one $(\mathbf{1 g}), 83 \%$ yield, $1.1 \mathrm{~g}$, brown solid, $R_{\mathrm{f}}=0.5$ (petroleum ether: EtOAc $\left.=8: 2\right)$; mp: 70-72 ${ }^{\circ} \mathrm{C} ;{ }^{1} \mathrm{H}$ NMR $\left(300 \mathrm{MHz}, \mathrm{CDCl}_{3}\right) \delta 7.69(\mathrm{~d}$, $J=8.0 \mathrm{~Hz}, 1 \mathrm{H}), 7.38(\mathrm{~d}, J=3.6 \mathrm{~Hz}, 2 \mathrm{H}), 7.28(\mathrm{~s}, 1 \mathrm{H}), 7.22-7.09(\mathrm{~m}, 1 \mathrm{H}), 4.07(\mathrm{~s}, 3 \mathrm{H}), 2.61$ $(\mathrm{s}, 3 \mathrm{H}) ;{ }^{13} \mathrm{C} \mathrm{NMR}\left(101 \mathrm{MHz}, \mathrm{CDCl}_{3}\right) \delta 191.7,140.1,134.9,125.9,125.8,122.9,120.7$, 112.0, 110.4, 32.2, 28.0; IR (KBr): 2949, 2840, 1690, 1600, 1257, 1166, $757 \mathrm{~cm}^{-1}$; MS (ESI): HRMS (ESI): $m / z$ calcd for $\mathrm{C}_{11} \mathrm{H}_{13} \mathrm{NO}(\mathrm{M}+\mathrm{H})^{+}: 174.0919$, found: 174.0910 . 


\section{1-(1-Methyl-1H-indol-2-yl)butan-1-one (1h):}

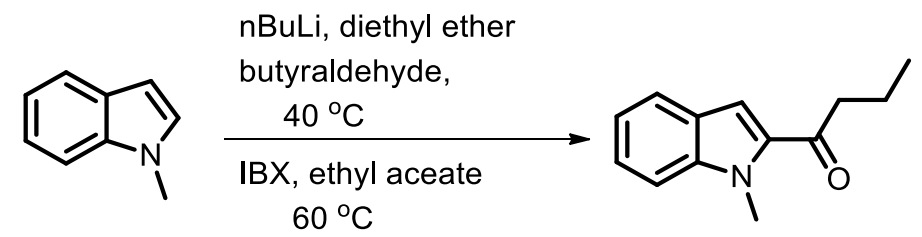

Following the general procedure A, $N$-methylindole $(1 \mathrm{~g}, 7.63 \mathrm{mmol})$ in diethyl ether was allowed to react with butyraldehyde $(0.7 \mathrm{~mL}, 8.39 \mathrm{mmol})$ in the presence of $\mathrm{n}-\mathrm{BuLi}(6.1 \mathrm{~mL}, 2.5 \mathrm{M})$ to get crude 1-(1-methyl-1H-indol-2-yl)butan-1-ol, which was further oxidized with IBX (3.2 g, 11.42 mmol) to obtain 1-(1-methyl-1H-indol-2-yl)butan-1-one (1h), $1.2 \mathrm{~g}, 78 \%$ yield, off-white solid, $R_{\mathrm{f}}=0.5$ (petroleum ether : EtOAc $\left.=8: 2\right) ; \mathrm{mp}: 81-83{ }^{\circ} \mathrm{C} ;{ }^{1} \mathrm{H}$ NMR $\left(300 \mathrm{MHz}, \mathrm{CDCl}_{3}\right) \delta 7.69(\mathrm{~d}$, $J=8.0 \mathrm{~Hz}, 1 \mathrm{H}), 7.37$ (d, $J=3.8 \mathrm{~Hz}, 2 \mathrm{H}), 7.29$ (s, 1H), 7.21-7.10 (m, 1H), 4.07 (s, 3H), 2.94 $(\mathrm{t}, J=7.4 \mathrm{~Hz}, 2 \mathrm{H}), 1.97-1.69(\mathrm{~m}, 2 \mathrm{H}), 1.02(\mathrm{t}, J=7.4 \mathrm{~Hz}, 3 \mathrm{H}) ;{ }^{13} \mathrm{C} \mathrm{NMR}\left(101 \mathrm{MHz}, \mathrm{CDCl}_{3}\right)$ $\delta 194.7,140.1,135.0,125.8,122.9,120.7,111.2,110.4,42.0,32.2,18.7,14.0$; IR (KBr): 3057, 2960, 1668, 1394, 1175, 1002, $749 \mathrm{~cm}^{-1}$; HRMS (ESI): $\mathrm{m} / z$ calcd for $\mathrm{C}_{13} \mathrm{H}_{16} \mathrm{NO}(\mathrm{M}+\mathrm{H})^{+}$: 202.1232, found: 202.1227 .

\section{Cyclopropyl(1-methyl-1H-indol-2-yl)methanone(1i):}

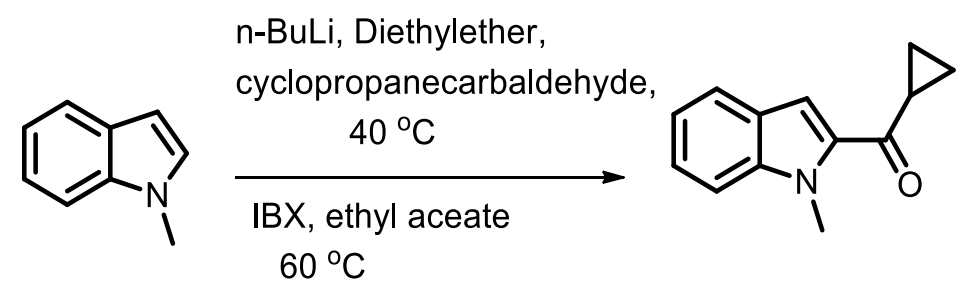

Following the general procedure $\mathrm{A}, \mathrm{N}$-methylindole $(1 \mathrm{~g}, 7.63 \mathrm{mmol})$ in diethyl ether was allowed to react with cyclopropanecarbaldehyde $(587 \mathrm{mg}, 8.39 \mathrm{mmol})$ in the presence of $\mathrm{n}-\mathrm{BuLi}(6.1 \mathrm{~mL}$, $2.5 \mathrm{M})$ to get crude cyclopropyl(1-methyl-1H-indol-2-yl)methanol, which was further oxidized with IBX (3.2 g, $11.42 \mathrm{mmol})$ to obtain cyclopropyl(1-methyl-1H-indol-2-yl)methanone (1i), $1.2 \mathrm{~g}, 79 \%$ yield, yellow oil, $R_{f}=0.5$ (petroleum ether: EtOAc $\left.=8: 2\right) ;{ }^{1} \mathrm{H}$ NMR $(500 \mathrm{MHz}$, $\left.\mathrm{CDCl}_{3}\right) \delta$ 7.74-7.68 (m, 1H), $7.43(\mathrm{~s}, 1 \mathrm{H}), 7.41-7.34(\mathrm{~m}, 2 \mathrm{H}), 7.16-7.12(\mathrm{~m}, 1 \mathrm{H}), 4.06(\mathrm{~s}$, $3 \mathrm{H}), 2.78-2.61(\mathrm{~m}, 1 \mathrm{H}), 1.27-1.18(\mathrm{~m}, 2 \mathrm{H}), 1.09-0.92(\mathrm{~m}, 2 \mathrm{H}) ;{ }^{13} \mathrm{C} \mathrm{NMR}\left(101 \mathrm{MHz}, \mathrm{CDCl}_{3}\right)$ $\delta 193.7,140.1,135.8,126.0,125.7,122.8,120.6,111.2,110.4,32.2,18.7,11.0 ;$ IR (neat): 3058, 3012, 2933, 1653, 1200, 997, $747 \mathrm{~cm}^{-1}$; HRMS (ESI): $\mathrm{m} / z$ calcd for $\mathrm{C}_{13} \mathrm{H}_{14} \mathrm{NO}(\mathrm{M}+\mathrm{H})^{+}$: 200.1075, found: 200.1069 .

\section{Cyclopentyl(1-methyl-1H-indol-2-yl)methanone(1j):}




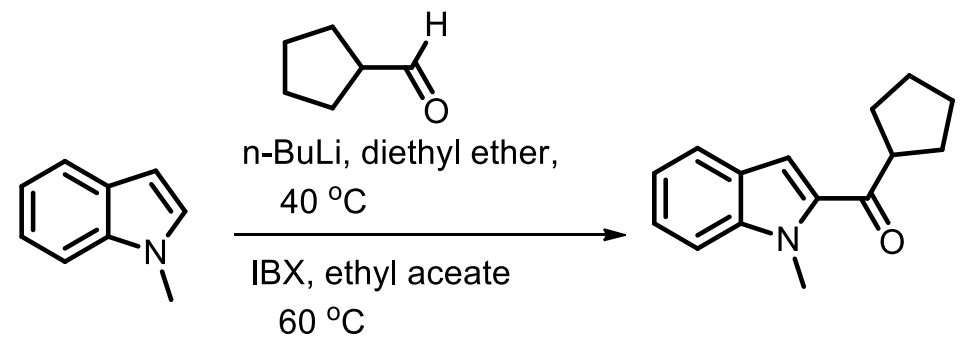

Following the general procedure A, $N$-methylindole $(1 \mathrm{~g}, 7.63 \mathrm{mmol})$ in diethyl ether was allowed to react with cyclopentanecarbaldehyde $(748 \mathrm{mg}, 8.39 \mathrm{mmol})$ in the presence of $\mathrm{n}-\mathrm{BuLi}(6.1 \mathrm{~mL}$, $2.5 \mathrm{M})$ to get crude cyclopentyl(1-methyl-1H-indol-2-yl)methanol, which was further oxidized with IBX (3.2 g, $11.42 \mathrm{mmol})$ to obtain cyclopentyl(1-methyl- $1 H$-indol-2-yl)methanone (1j), $1.2 \mathrm{~g}, 75 \%$ yield, off-white solid, $R_{\mathrm{f}}=0.5$ (petroleum ether: EtOAc $=8: 2$ ); mp: $79-81{ }^{\circ} \mathrm{C} ;{ }^{1} \mathrm{H}$ NMR (500 MHz, $\left.\mathrm{CDCl}_{3}\right) \delta$ 7.73-7.65 (m, 1H), 7.42-7.34 (m, 2H), $7.31(\mathrm{~s}, 1 \mathrm{H}), 7.19-7.11$ $(\mathrm{m}, 1 \mathrm{H}), 4.09(\mathrm{~d}, J=3.4 \mathrm{~Hz}, 3 \mathrm{H}), 3.78-3.65(\mathrm{~m}, 1 \mathrm{H}), 1.99-1.89(\mathrm{~m}, 4 \mathrm{H}), 1.82-1.73(\mathrm{~m}, 2 \mathrm{H})$, $1.72-1.62(\mathrm{~m}, 2 \mathrm{H}) ;{ }^{13} \mathrm{C}$ NMR $\left(101 \mathrm{MHz}, \mathrm{CDCl}_{3}\right) \delta 197.4,140.1,135.1,125.9,125.7,122.8$, 120.6, 111.2, 110.4, 48.1, 32.3, 30.8, 26.5; IR (KBr): 3050, 2840, 1690, 1600, 1257, 1166, 757 $\mathrm{cm}^{-1}$; HRMS (ESI): $m / z$ calcd for $\mathrm{C}_{15} \mathrm{H}_{18} \mathrm{NO}(\mathrm{M}+\mathrm{H})^{+}: 228.1388$, found: 228.1386 .

\section{B. General procedure for synthesis of 3-hydroxycarbazoles:}

\section{General reaction:}<smiles>[R]C#CC([R5])O</smiles>

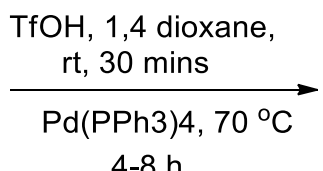<smiles>[R]c1c(O)c([R])c2c3ccccc3n([R1])c2c1[R]</smiles>

To a stirred solution of 2-carbonylindole $1(0.5 \mathrm{mmol})$ and propargylic alcohol $2(0.5 \mathrm{mmol})$ in $3 \mathrm{~mL}$ of dioxane in a reaction vial was added $\mathrm{TfOH}(20 \mathrm{~mol} \%)$ at room temperature and stirred for $30 \mathrm{~min}$. Then, $\mathrm{Pd}\left(\mathrm{PPh}_{3}\right)_{4}(5 \mathrm{~mol} \%)$ was added and stirred at $80{ }^{\circ} \mathrm{C}$ (oil bath temperature) for 6-8 h. After completion of the reaction (monitored by TLC) the reaction mixture was concentrated under reduced pressure. The crude product was purified by column chromatography on silica gel by using EtOAc: hexanes as an eluent to afford the corresponding 3-hydroxycarbazole 3.

\section{9-Methyl-2,4-diphenyl-9H-carbazol-3-ol (3a):}




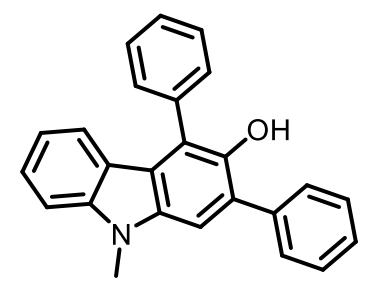

Following the general procedure $\mathrm{B}$, to the solution of 1-methyl- $1 H$-indole-2-carbaldehyde $1 \mathrm{a}$ (80 $\mathrm{mg}, 0.5 \mathrm{mmol})$ and 1,3-diphenylprop-2-yn-1-ol 2a (104 mg, $0.5 \mathrm{mmol})$ in $3 \mathrm{~mL}$ of dioxane, TfOH $(0.01 \mathrm{~mL}, 20 \mathrm{~mol} \%)$ was added at room temperature and stirred for $30 \mathrm{~min}$. Then, $\mathrm{Pd}\left(\mathrm{PPh}_{3}\right)_{4}(29$ $\mathrm{mg}, 5 \mathrm{~mol} \%$ ) was added and stirred at $80{ }^{\circ} \mathrm{C}$ for $8 \mathrm{~h}$. After completion of the reaction (monitored by TLC), the reaction mixture was concentrated under reduced pressure. The crude product was purified by column chromatography on silica gel (EtOAc-hexanes) to afford the product 9-methyl-2,4-diphenyl-9H-carbazol-3-ol (3a), $140 \mathrm{mg}, 80 \%$ yield, off-white solid; $R_{f}=0.4$ (hexanes: EtOAc $\left.=9: 1\right) ; \mathrm{mp} 184-186{ }^{\circ} \mathrm{C} ;{ }^{1} \mathrm{H}$ NMR $\left(400 \mathrm{MHz}, \mathrm{CDCl}_{3}\right) \delta 7.71$ $(\mathrm{d}, J=7.4 \mathrm{~Hz}, 2 \mathrm{H}), 7.62-7.55(\mathrm{~m}, 5 \mathrm{H}), 7.53-7.45(\mathrm{~m}, 2 \mathrm{H}), 7.41-7.32(\mathrm{~m}, 4 \mathrm{H}), 6.99(\mathrm{~d}, J=$ $7.8 \mathrm{~Hz}, 1 \mathrm{H}), 6.88(\mathrm{t}, J=7.0 \mathrm{~Hz}, 1 \mathrm{H}), 4.96(\mathrm{~s}, 1 \mathrm{H}), 3.85(\mathrm{~s}, 3 \mathrm{H}) ;{ }^{13} \mathrm{C} \mathrm{NMR}\left(101 \mathrm{MHz}, \mathrm{CDCl}_{3}\right)$ $\delta 143.1,141.9,139.0,135.9,135.5,130.5,129.7,129.6,128.6(2 \mathrm{C}), 127.5,127.3,125.6$, 122.3, 122.0, 122.0, 120.9, 118.2, 109.4, 108.3, 29.2; IR (KBr): 3556, 3018, 2923, 1586, 1249, 1024, $752 \mathrm{~cm}^{-1}$; HRMS (ESI): $\mathrm{m} / z$ calcd for $\mathrm{C}_{25} \mathrm{H}_{20} \mathrm{NO}(\mathrm{M}+\mathrm{H})^{+}$: 350.1545, found: 350.1544 .

\section{9-Methyl-2-phenyl-4-(p-tolyl)-9H-carbazol-3-ol (3b):}

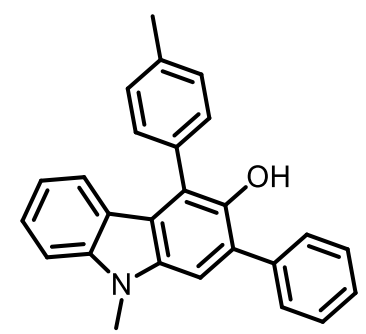

Following the general procedure $\mathrm{B}$, to the solution of 1 -methyl- $1 H$-indole-2-carbaldehyde $1 \mathbf{a}$ (80 $\mathrm{mg}, 0.5 \mathrm{mmol})$ and 3-phenyl-1-(p-tolyl)prop-2-yn-1-ol $2 \mathbf{b}(111 \mathrm{mg}, 0.5 \mathrm{mmol})$ in $3 \mathrm{~mL}$ of dioxane, $\mathrm{TfOH}(0.01 \mathrm{~mL}, 20 \mathrm{~mol} \%)$ was added at room temperature and stirred for $30 \mathrm{~min}$. Then, $\mathrm{Pd}\left(\mathrm{PPh}_{3}\right)_{4}(29 \mathrm{mg}, 5 \mathrm{~mol} \%)$ was added to the reaction mixture and stirred at $80{ }^{\circ} \mathrm{C}$ for $8 \mathrm{~h}$. After completion of the reaction (monitored by TLC), the reaction mixture was concentrated under reduced pressure. The crude product was purified by column chromatography on silica gel (EtOAc-hexanes) to afford the product 9-methyl-2-phenyl-4-(p-tolyl)-9H-carbazol-3ol(3b), $142 \mathrm{mg}, 78 \%$ yield, brown solid; $R_{f}=0.5$ (hexanes: EtOAc = 9:1); mp: $172-174{ }^{\circ} \mathrm{C}$; ${ }^{1} \mathrm{H}$ NMR (300 MHz, $\left.\mathrm{CDCl}_{3}\right) \delta 7.71(\mathrm{~d}, J=7.2 \mathrm{~Hz}, 2 \mathrm{H}), 7.54-7.45(\mathrm{~m}, 4 \mathrm{H}), 7.45-7.32(\mathrm{~m}$, $6 \mathrm{H}), 7.06(\mathrm{~d}, J=7.9 \mathrm{~Hz}, 1 \mathrm{H}), 6.95-6.85(\mathrm{~m}, 1 \mathrm{H}), 4.99(\mathrm{~s}, 1 \mathrm{H}), 3.85(\mathrm{~s}, 3 \mathrm{H}), 2.52(\mathrm{~s}, 3 \mathrm{H}) ;{ }^{13} \mathrm{C}$ 
NMR $\left(101 \mathrm{MHz}, \mathrm{CDCl}_{3}\right) \delta 143.2,141.8,139.1,138.2,135.9,132.0,130.2,130.2,129.7$ (2C), 128.4, 127.3, 127.2, 125.4, 122.3, 122.0, 121.0, 118.1, 109.1, 108.1, 29.1, 21.4; IR (KBr): 3553, 3018, 2925, 1700, 1257, 1024, $752 \mathrm{~cm}^{-1}$; HRMS (ESI): $\mathrm{m} / z$ calcd for $\mathrm{C}_{26} \mathrm{H}_{22} \mathrm{NO}(\mathrm{M}+\mathrm{H})^{+}:$364.1701, found: 364.1700 .

\section{4-(4-Methoxyphenyl)-9-methyl-2-phenyl-9H-carbazol-3-ol (3c):}

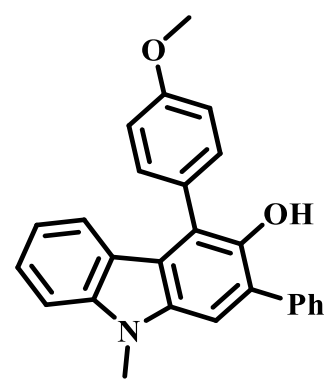

Following the general procedure $\mathrm{B}$, to the solution of 1-methyl-1H-indole-2-carbaldehyde 1a (80 $\mathrm{mg}, 0.5 \mathrm{mmol})$ and 1-(4-methoxyphenyl)-3-phenylprop-2-yn-1-ol 2c (119 mg, $0.5 \mathrm{mmol})$ in $3 \mathrm{~mL}$ of dioxane, TfOH $(0.01 \mathrm{~mL}, 20 \mathrm{~mol} \%)$ was added at room temperature and stirred for $30 \mathrm{~min}$. Then, $\mathrm{Pd}\left(\mathrm{PPh}_{3}\right)_{4}(29 \mathrm{mg}, 5 \mathrm{~mol} \%)$ was added to the reaction mixture and stirred at $80{ }^{\circ} \mathrm{C}$ for $8 \mathrm{~h}$. After completion of the reaction (monitored by TLC), the reaction mixture was concentrated under reduced pressure. The crude product was purified by column chromatography on silica gel (EtOAc-hexanes) to afford the product 4-(4-methoxyphenyl)-9methyl-2-phenyl-9H-carbazol-3-ol (3c), $152 \mathrm{mg}, 80 \%$ yield, off-white solid; $R_{f}=0.4$ (hexanes: EtOAc = 9:1); mp:194-196 ${ }^{\circ} \mathrm{C} ;{ }^{1} \mathrm{H}$ NMR $\left(400 \mathrm{MHz}, \mathrm{CDCl}_{3}\right) \delta 7.70(\mathrm{~d}, J=7.3 \mathrm{~Hz}$, 2H), 7.55-7.45 (m, 4H), 7.42-7.30 (m, 4H), 7.19-7.12 (m, 2H), 7.07 (d, J = 7.9 Hz, 1H), 6.95-6.89 (m, 1H), 4.99 (s, 1H), $3.94(\mathrm{~s}, 3 \mathrm{H}), 3.84(\mathrm{~s}, 3 \mathrm{H}) ;{ }^{13} \mathrm{C} \mathrm{NMR}\left(101 \mathrm{MHz}, \mathrm{CDCl}_{3}\right) \delta$ $159.8,143.4,141.9,139.1,135.8,131.7,129.7,128.5,127.4,127.3,127.1,125.5,122.3$, 122.0, 121.5, 121.2, 118.2, 115.0, 109.2, 108.2, 55.4, 29.2; IR (KBr):3536, 3017, 2944, 1514, 1248, $757 \mathrm{~cm}^{-1}$; HRMS (ESI): $\mathrm{m} / z$ calcd for $\mathrm{C}_{26} \mathrm{H}_{22} \mathrm{NO}_{2}(\mathrm{M}+\mathrm{H})^{+}: 380.1651$, found: 380.1635 .

\section{4-(4-Bromophenyl)-9-methyl-2-phenyl-9H-carbazol-3-ol (3d):}

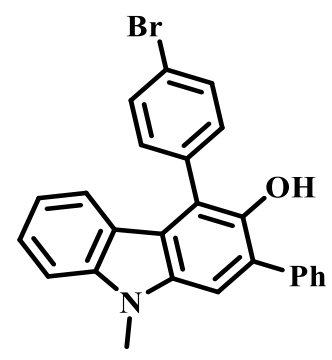

Following the general procedure $\mathrm{B}$, to the solution of 1-methyl- $1 H$-indole-2-carbaldehyde $1 \mathbf{a}$ (80 $\mathrm{mg}, 0.5 \mathrm{mmol}$ ) and 3-(4-bromophenyl)-1-phenylprop-2-yn-1-ol $2 \mathbf{d}(143 \mathrm{mg}, 0.5 \mathrm{mmol})$ in $3 \mathrm{~mL}$ 
of dioxane, TfOH $(0.01 \mathrm{~mL}, 20 \mathrm{~mol} \%)$ was added at room temperature and stirred for $30 \mathrm{~min}$. Then, $\mathrm{Pd}\left(\mathrm{PPh}_{3}\right)_{4}(29 \mathrm{mg}, 5 \mathrm{~mol} \%)$ was added to the reaction mixture and stirred at $80{ }^{\circ} \mathrm{C}$ for 8 h. After completion of the reaction (monitored by TLC), the reaction mixture was concentrated under reduced pressure. The crude product was purified by column chromatography on silica gel (EtOAc-hexanes) to afford the product 4-(4-bromophenyl)-9methyl-2-phenyl-9H-carbazol-3-ol (3d), $170 \mathrm{mg}$, 79\% yield, off- white solid; $R_{f}=0.4$ (hexanes: EtOAc = 9:1); mp: $232-234{ }^{\circ} \mathrm{C} ;{ }^{1} \mathrm{H}$ NMR $\left(400 \mathrm{MHz}, \mathrm{CDCl}_{3}\right) \delta 7.74(\mathrm{~d}, J=8.2 \mathrm{~Hz}$, 2H), $7.66(\mathrm{~d}, J=7.3 \mathrm{~Hz}, 2 \mathrm{H}), 7.56-7.46(\mathrm{~m}, 4 \mathrm{H}), 7.44-7.31(\mathrm{~m}, 4 \mathrm{H}), 7.06(\mathrm{~d}, J=7.8 \mathrm{~Hz}$, $1 \mathrm{H}), 6.93(\mathrm{t}, J=7.0 \mathrm{~Hz}, 1 \mathrm{H}), 4.89(\mathrm{~s}, 1 \mathrm{H}), 3.85$ (s, 3H); ${ }^{13} \mathrm{C} \mathrm{NMR}\left(101 \mathrm{MHz}, \mathrm{CDCl}_{3}\right) \delta$ 143.0, 142.0, 138.6, 135.9, 134.8, 132.5, 132.3, 129.7, 128.9 (2C), 127.7, 127.6, 125.8, 122.5, 122.0, 122.0, 120.8, 118.4, 109.6, 108.4, 29.2; IR (KBr): 3540, 3472, 2924, 2857, 1715, 1468, $744 \mathrm{~cm}^{-1}$; HRMS (ESI): $\mathrm{m} / z$ calcd for $\mathrm{C}_{25} \mathrm{H}_{19} \mathrm{BrNO}(\mathrm{M}+\mathrm{H})^{+}$: 428.0650, found: 428.0651 .

\section{9-Methyl-4-(4-nitrophenyl)-2-phenyl-9H-carbazol-3-ol (3e):}

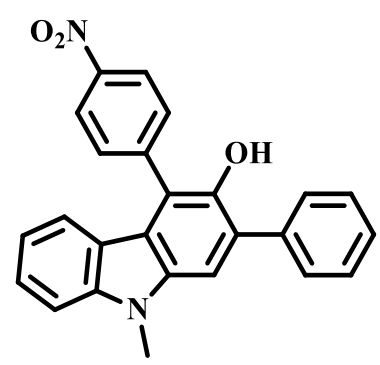

Following the general procedure $\mathrm{B}$, to the solution of 1-methyl-1H-indole-2-carbaldehyde 1a (80 $\mathrm{mg}, 0.5 \mathrm{mmol}$ ) and 3-(4-nitrophenyl)-1-phenylprop-2-yn-1-ol $2 \mathrm{e}$ (127 mg, $0.5 \mathrm{mmol})$ in $3 \mathrm{~mL}$ of dioxane, $\mathrm{TfOH}(0.01 \mathrm{~mL}, 20 \mathrm{~mol} \%)$ was added at room temperature and stirred for $30 \mathrm{~min}$. Then, $\mathrm{Pd}\left(\mathrm{PPh}_{3}\right)_{4}(29 \mathrm{mg}, 5 \mathrm{~mol} \%)$ was added to the reaction mixture and stirred at $80{ }^{\circ} \mathrm{C}$ for $8 \mathrm{~h}$. After completion of the reaction (monitored by TLC), the reaction mixture was concentrated under reduced pressure. The crude product was purified by column chromatography on silica gel (EtOAc-hexanes) to afford the product 9-methyl-4-(4-nitrophenyl)-2-phenyl-9Hcarbazol-3-ol (3e), $170 \mathrm{mg}$, 85\% yield, off-white solid; $\mathrm{R}_{f}=0.3$ (hexanes: EtOAc $=9: 1$ ); mp: 164-166 ${ }^{\circ} \mathrm{C} ;{ }^{1} \mathrm{H}$ NMR $\left(500 \mathrm{MHz}, \mathrm{CDCl}_{3}\right) \delta 8.48-8.40(\mathrm{~m}, 2 \mathrm{H}), 7.84-7.78$ (m, 2H), 7.67-7.60 $(\mathrm{m}, 2 \mathrm{H}), 7.58-7.51(\mathrm{~m}, 2 \mathrm{H}), 7.49-7.33(\mathrm{~m}, 4 \mathrm{H}), 7.02(\mathrm{~d}, J=8.0 \mathrm{~Hz}, 1 \mathrm{H}), 6.96-6.88(\mathrm{~m}, 1 \mathrm{H})$, 4.95 (s, 1H), 3.87 (s, 3H); ${ }^{13} \mathrm{C}$ NMR $\left(126 \mathrm{MHz}, \mathrm{CDCl}_{3}\right) \delta 147.6,143.9,142.7,142.0,137.8$, 136.0, 131.7, 129.6, 129.4, 128.2, 127.7, 126.1, 124.0, 121.8, 121.7, 120.6, 120.1, 118.6, 110.0, 108.6, 29.2; IR (KBr): 3687, 3021, 2891, 2405, 1215, $739 \mathrm{~cm}^{-1}$; HRMS (ESI): $\mathrm{m} / \mathrm{z}$ calcd for $\mathrm{C}_{25} \mathrm{H}_{19} \mathrm{~N}_{2} \mathrm{O}_{3}(\mathrm{M}+\mathrm{H})^{+}:$395.1396, found: 395.1393 . 


\section{4-(3-Hydroxy-9-methyl-2-phenyl-9H-carbazol-4-yl)benzonitrile (3f):}

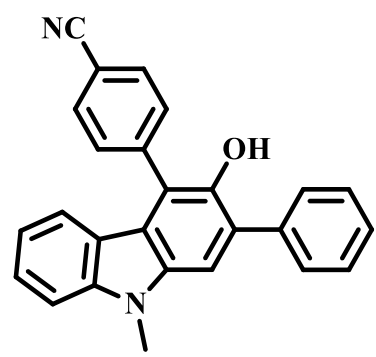

Following the general procedure B, to the solution of 1-methyl-1H-indole-2-carbaldehyde 1a (80 $\mathrm{mg}, 0.5 \mathrm{mmol}$ ) and 4-(3-hydroxy-3-phenylprop-1-yn-1-yl)benzonitrile $2 \mathbf{f}$ (117 mg, $0.5 \mathrm{mmol})$ in 3 $\mathrm{mL}$ of dioxane, $\mathrm{TfOH}(0.01 \mathrm{~mL}, 20 \mathrm{~mol} \%)$ was added at room temperature and stirred for $30 \mathrm{~min}$. Then, $\mathrm{Pd}\left(\mathrm{PPh}_{3}\right)_{4}(29 \mathrm{mg}, 5 \mathrm{~mol} \%)$ was added to the reaction mixture and stirred at $80{ }^{\circ} \mathrm{C}$ for 8 h. After completion of the reaction (monitored by TLC), the reaction mixture was concentrated under reduced pressure. The crude product was purified by column chromatography on silica gel (EtOAc-hexanes) to afford the 4-(3-hydroxy-9-methyl-2phenyl-9H-carbazol-4-yl)benzonitrile (3f), $140 \mathrm{mg}, 74 \%$ yield, off-white solid; $R_{f}=0.4$ (hexanes: EtOAc = 9:1); mp: $235-237{ }^{\circ} \mathrm{C} ;{ }^{1} \mathrm{H}$ NMR $\left(500 \mathrm{MHz}, \mathrm{CDCl}_{3}\right) \delta 7.88(\mathrm{~d}, J=8.2 \mathrm{~Hz}$, 2H), $7.75(\mathrm{~d}, J=8.2 \mathrm{~Hz}, 2 \mathrm{H}), 7.67-7.60(\mathrm{~m}, 2 \mathrm{H}), 7.57-7.52(\mathrm{~m}, 2 \mathrm{H}), 7.48-7.33(\mathrm{~m}, 4 \mathrm{H})$, $6.99(\mathrm{~d}, J=7.9 \mathrm{~Hz}, 1 \mathrm{H}), 6.96-6.90(\mathrm{~m}, 1 \mathrm{H}), 4.92(\mathrm{~s}, 1 \mathrm{H}), 3.86(\mathrm{~s}, 3 \mathrm{H}) ;{ }^{13} \mathrm{C} \mathrm{NMR}(101 \mathrm{MHz}$, $\left.\mathrm{CDCl}_{3}\right) \delta 142.7,142.0,141.8,137.9,135.9,132.6,131.5,129.5,129.3,128.1,127.6,126.0$, 121.8, 120.6, 120.5, 119.0, 118.5, 111.8, 109.7, 108.6, 29.2; IR (KBr): 3693, 3022, 2361, 1533, 1215, $758 \mathrm{~cm}^{-1}$; HRMS (ESI): $\mathrm{m} / z$ calcd for $\mathrm{C}_{26} \mathrm{H}_{19} \mathrm{~N}_{2} \mathrm{O}(\mathrm{M}+\mathrm{H})^{+}:$375.1497, found: 375.1489 .

\section{9-Methyl-4-(naphthalen-1-yl)-2-phenyl-9H-carbazol-3-ol (3g):}

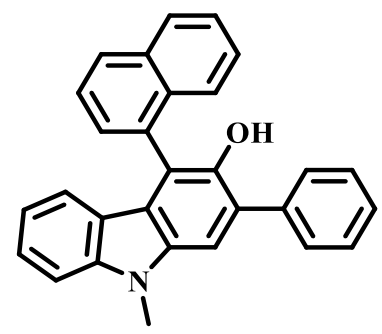

Following the general procedure $\mathrm{B}$, to the solution of 1-methyl- $1 H$-indole-2-carbaldehyde $1 \mathbf{a}$ (80 mg, $0.5 \mathrm{mmol}$ ) and 4-(3-hydroxy-3-(naphthalen-1-yl)prop-1-yn-1-yl)benzonitrile $2 \mathrm{~g}$ (142 mg, 0.5 $\mathrm{mmol})$ in $3 \mathrm{~mL}$ of dioxane, $\mathrm{TfOH}(0.01 \mathrm{~mL}, 20 \mathrm{~mol} \%)$ was added at room temperature and stirred for $30 \mathrm{~min}$. Then, $\mathrm{Pd}\left(\mathrm{PPh}_{3}\right)_{4}(29 \mathrm{mg}, 5 \mathrm{~mol} \%)$ was added and stirred at $80{ }^{\circ} \mathrm{C}$ for $8 \mathrm{~h}$. 
After the completion of reaction (monitored by TLC), the reaction mixture was concentrated under reduced pressure. The crude product was purified by column chromatography on silica gel (EtOAc-hexanes) to afford the product 9-methyl-4-(naphthalen-1-yl)-2-phenyl-9Hcarbazol-3-ol (3g), $160 \mathrm{mg}, 80 \%$ yield, off-white solid; $R_{f}=0.4$ (hexanes: EtOAc $=9: 1$ ); mp: 160-162 ${ }^{\circ} \mathrm{C} ;{ }^{1} \mathrm{H}$ NMR (400 MHz, $\left.\mathrm{CDCl}_{3}\right) \delta 8.12-7.96$ (m, 2H), 7.80-7.68 (m, 4H), 7.62 (d, J $=8.3 \mathrm{~Hz}, 1 \mathrm{H}), 7.57-7.44(\mathrm{~m}, 4 \mathrm{H}), 7.41-7.29(\mathrm{~m}, 4 \mathrm{H}), 6.68(\mathrm{t}, J=7.0 \mathrm{~Hz}, 1 \mathrm{H}), 6.45(\mathrm{~d}, J=$ $7.8 \mathrm{~Hz}, 1 \mathrm{H}), 4.84(\mathrm{~s}, 1 \mathrm{H}), 3.90(\mathrm{~s}, 3 \mathrm{H}) ;{ }^{13} \mathrm{C} \mathrm{NMR}\left(75 \mathrm{MHz}, \mathrm{CDCl}_{3}\right) \delta$ 143.8, 141.9, 139.0, $135.9,134.2$, 132.9, 132.3, 129.8, 129.1, 128.5(2C), 128.4, 127.5, 127.3, 126.8, 126.5, 126.1, 125.8, 125.5, 122.1, 121.9, 121.7, 119.7, 118.3, 109.7, 108.1, 29.2; IR (KBr): 3694, 3539, 2936, 1647, 1215, $757 \mathrm{~cm}^{-1}$; HRMS (ESI): $\mathrm{m} / \mathrm{z}$ calcd for $\mathrm{C}_{29} \mathrm{H}_{22} \mathrm{NO}(\mathrm{M}+\mathrm{H})^{+}: 400.1701$, found: 400.1695 .

\section{9-Methyl-4-phenyl-2-(p-tolyl)-9H-carbazol-3-ol (3h):}

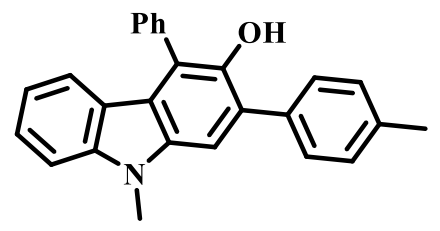

Following the general procedure $\mathrm{B}$, to the solution of 1-methyl-1H-indole-2-carbaldehyde 1a (80 $\mathrm{mg}, 0.5 \mathrm{mmol})$ and 1-phenyl-3-(p-tolyl)prop-2-yn-1-ol $\mathbf{2 h}(111 \mathrm{mg}, 0.5 \mathrm{mmol})$ in $3 \mathrm{~mL}$ of dioxane, $\mathrm{TfOH}(0.01 \mathrm{~mL}, 20 \mathrm{~mol} \%)$ was added at room temperature and stirred for $30 \mathrm{~min}$. Then, $\mathrm{Pd}\left(\mathrm{PPh}_{3}\right)_{4}(29 \mathrm{mg}, 5 \mathrm{~mol} \%)$ was added to the reaction mixture and stirred at $80{ }^{\circ} \mathrm{C}$ for $7 \mathrm{~h}$. After completion of the reaction (monitored by TLC), the reaction mixture was concentrated under reduced pressure. The crude product was purified by column chromatography on silica gel (EtOAc-hexanes) to afford the product 9-methyl-4-phenyl-2-(p-tolyl)-9H-carbazol-3-ol (3h), $140 \mathrm{mg}, 77 \%$ yield, off-white solid; $R_{f}=0.4$ (hexanes: EtOAc $=9: 1$ ); mp: $180-182{ }^{\circ} \mathrm{C}$; ${ }^{1} \mathrm{H}$ NMR $\left(300 \mathrm{MHz}, \mathrm{CDCl}_{3}\right) \delta$ 7.58-7.47 (m, 6H), 7.34-7.25 (m, 2H), 7.23-7.10 (m, 3H), 7.09-6.96 (m, 1H), 6.92 (d, $J=7.9 \mathrm{~Hz}, 1 \mathrm{H}), 6.86-6.76(\mathrm{~m}, 1 \mathrm{H}), 4.88$ (s, 1H), 3.77 (s, 3H), $2.36(\mathrm{~s}, 3 \mathrm{H}) ;{ }^{13} \mathrm{C} \mathrm{NMR}\left(126 \mathrm{MHz}, \mathrm{CDCl}_{3}\right) \delta 143.1,141.9,137.2,135.9,135.9,135.6,130.4$, $129.5,129.4,129.3,128.4,127.7,127.5,125.4,122.3,122.0,120.8,118.2,109.2,108.2$, 29.2, 21.3; IR (KBr): 3684, 3023, 2404, 1532, 1344, 1215, $739 \mathrm{~cm}^{-1}$; HRMS (ESI): $\mathrm{m} / z$ calcd for $\mathrm{C}_{26} \mathrm{H}_{22} \mathrm{NO}(\mathrm{M}+\mathrm{H})^{+}: 364.1701$, found: 364.1699 .

\section{2-(4-Methoxyphenyl)-9-methyl-4-phenyl-9H-carbazol-3-ol (3i):}

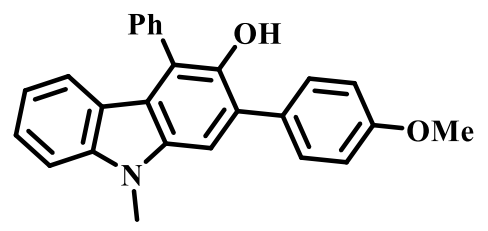


Following the general procedure $\mathrm{B}$, to the solution of 1-methyl- $1 H$-indole-2-carbaldehyde $1 \mathbf{a}$ (80 $\mathrm{mg}, 0.5 \mathrm{mmol}$ ) and 3-(4-methoxyphenyl)-1-phenylprop-2-yn-1-ol $2 \mathbf{i}$ (119 mg, $0.5 \mathrm{mmol})$ in $3 \mathrm{~mL}$ of dioxane, TfOH $(0.01 \mathrm{~mL}, 20 \mathrm{~mol} \%)$ was added at room temperature and stirred for $30 \mathrm{~min}$. Then, $\mathrm{Pd}\left(\mathrm{PPh}_{3}\right)_{4}(29 \mathrm{mg}, 5 \mathrm{~mol} \%)$ was added to the reaction mixture and stirred at $80{ }^{\circ} \mathrm{C}$ for $7 \mathrm{~h}$. After completion of the reaction (monitored by TLC), the reaction mixture was concentrated under reduced pressure. The crude product was purified by column chromatography on silica gel (EtOAc-hexanes) to afford the product 2-(4-methoxyphenyl)-9methyl-4-phenyl-9H-carbazol-3-ol (3i), $130 \mathrm{mg}$, 68\% yield, off-white solid; $R_{f}=0.3$ (hexanes: EtOAc = 9:1); mp: $188-190{ }^{\circ} \mathrm{C} ;{ }^{1} \mathrm{H}$ NMR $\left(400 \mathrm{MHz}, \mathrm{CDCl}_{3}\right) \delta 7.67-7.53(\mathrm{~m}, 7 \mathrm{H}), 7.39-7.31$ $(\mathrm{m}, 3 \mathrm{H}), 7.04(\mathrm{~d}, J=8.8 \mathrm{~Hz}, 2 \mathrm{H}), 6.98(\mathrm{~d}, J=7.9 \mathrm{~Hz}, 1 \mathrm{H}), 6.93-6.84(\mathrm{~m}, 1 \mathrm{H}), 4.94(\mathrm{~s}, 1 \mathrm{H})$, 3.88 (s, 3H), 3.85 (s, 3H); ${ }^{13} \mathrm{C}$ NMR (101 MHz, $\left.\mathrm{CDCl}_{3}\right) \delta 159.0,143.2,141.9,135.9,135.6$, 131.2 , 130.8, 130.4, 129.5, 128.5, 127.2, 125.4, 122.3, 121.9, 121.9, 120.6, 118.2, 114.1, 109.1, 108.2, 55.4, 29.2; IR (KBr): 3538, 2929, 1609, 1519, 1253, 1033, $744 \mathrm{~cm}^{-1}$; HRMS (ESI): $m / z$ calcd for $\mathrm{C}_{26} \mathrm{H}_{22} \mathrm{NO}_{2}(\mathrm{M}+\mathrm{H})^{+}: 380.1651$, found: 380.1635 .

\section{9-Methyl-4-phenyl-2-(4-(trifluoromethyl)phenyl)-9H-carbazol-3-ol (3j):}

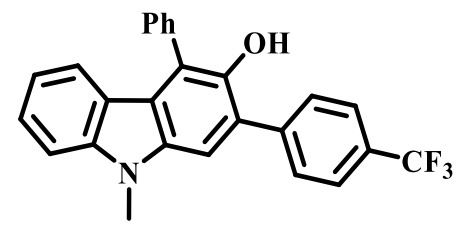

Following the general procedure $\mathrm{B}$, to the solution of 1-methyl- $1 H$-indole-2-carbaldehyde $1 \mathbf{a}$ (80 $\mathrm{mg}, 0.5 \mathrm{mmol}$ ) and 1-phenyl-3-(4-(trifluoromethyl)phenyl)prop-2-yn-1-ol 2j (138 mg, $0.5 \mathrm{mmol}$ ) in $3 \mathrm{~mL}$ of dioxane, TfOH $(0.01 \mathrm{~mL}, 20 \mathrm{~mol} \%)$ was added at room temperature and stirred for 30 min. Then, $\mathrm{Pd}\left(\mathrm{PPh}_{3}\right)_{4}(29 \mathrm{mg}, 5 \mathrm{~mol} \%)$ was added to the reaction mixture and stirred at $80{ }^{\circ} \mathrm{C}$ for $7 \mathrm{~h}$. After completion of the reaction (monitored by TLC), the reaction mixture was concentrated under reduced pressure. The crude product was purified by column chromatography on silica gel (EtOAc-hexanes) to afford the product 9-methyl-4-phenyl-2(4-(trifluoromethyl)phenyl)-9H-carbazol-3-ol (3j), $148 \mathrm{mg}, 70 \%$ yield, yellow oil; $R_{f}=0.4$ (hexanes: EtOAc = 9:1); ${ }^{1} \mathrm{H} \mathrm{NMR}\left(500 \mathrm{MHz}, \mathrm{CDCl}_{3}\right) \delta 7.87(\mathrm{~d}, J=8.0 \mathrm{~Hz}, 2 \mathrm{H}), 7.74(\mathrm{~d}, J=$ $8.1 \mathrm{~Hz}, 2 \mathrm{H}), 7.70-7.63(\mathrm{~m}, 2 \mathrm{H}), 7.63-7.57(\mathrm{~m}, 3 \mathrm{H}), 7.44-7.33(\mathrm{~m}, 3 \mathrm{H}), 6.99$ (d, J = 7.9 Hz, $1 \mathrm{H}), 6.92(\mathrm{t}, J=7.2 \mathrm{~Hz}, 1 \mathrm{H}), 4.97(\mathrm{~s}, 1 \mathrm{H}), 3.88(\mathrm{~s}, 3 \mathrm{H}) ;{ }^{13} \mathrm{C} \mathrm{NMR}\left(126 \mathrm{MHz}, \mathrm{CDCl}_{3}\right) \delta$ 143.1, 143.1, 142.1, 135.9, 134.7, 130.5, 130.1, 129.9, 129.0 (q, $J=32.13 \mathrm{~Hz}$ ), 129.0, 125.9, $125.9,125.2,125.2,124.4(\mathrm{q}, J=277.8 \mathrm{~Hz}), 122.0,121.4,118.5,109.5,108.4,29.2$; IR (neat): 3540, 3037, 2933, 1615, 1329, 1122, $750 \mathrm{~cm}^{-1}$; HRMS (ESI): $\mathrm{m} / \mathrm{z}$ calcd for $\mathrm{C}_{26} \mathrm{H}_{19} \mathrm{NOF}_{3}(\mathrm{M}+\mathrm{H})^{+}:$418.1419, found: 418.1415 . 


\section{9-Methyl-4-phenyl-2-propyl-9H-carbazol-3-ol (3k):}

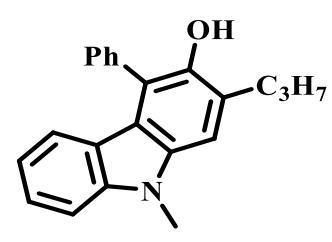

Following the general procedure $\mathrm{B}$, to the solution of 1-methyl-1H-indole-2-carbaldehyde $1 \mathbf{a}$ (80 $\mathrm{mg}, 0.5 \mathrm{mmol})$ and 1-phenylhex-2-yn-1-ol $2 \mathbf{k}(87 \mathrm{mg}, 0.5 \mathrm{mmol})$ in $3 \mathrm{~mL}$ of dioxane, $\mathrm{TfOH}(0.01$ $\mathrm{mL}, 20 \mathrm{~mol} \%$ ) was added at room temperature and stirred for $30 \mathrm{~min}$. Then, $\mathrm{Pd}\left(\mathrm{PPh}_{3}\right)_{4}(29 \mathrm{mg}, 5$ mol \%) was added to the reaction mixture and stirred at $80{ }^{\circ} \mathrm{C}$ for $7 \mathrm{~h}$. After completion of the reaction (monitored by TLC), the reaction mixture was concentrated under reduced pressure. The crude product was purified by column chromatography on silica gel (EtOAchexanes) to afford the product 9-methyl-4-phenyl-2-propyl-9H-carbazol-3-ol(3k), $115 \mathrm{mg}$, $72 \%$ yield, brown semisolid; $R_{f}=0.6$ (hexanes: EtOAc $\left.=9: 1\right) ;{ }^{1} \mathrm{H} \mathrm{NMR}\left(400 \mathrm{MHz}, \mathrm{CDCl}_{3}\right) \delta$ 7.69-7.58 (m, 2H), 7.59-7.48 (m, 3H), 7.38-7.27 (m, 2H), 7.19 (s, 1H), 6.93 (d, J = 7.9 Hz, $1 \mathrm{H}), 6.89-6.79(\mathrm{~m}, 1 \mathrm{H}), 4.80(\mathrm{~s}, 1 \mathrm{H}), 3.83(\mathrm{~s}, 3 \mathrm{H}), 2.91-2.75(\mathrm{~m}, 2 \mathrm{H}), 1.92-1.69(\mathrm{~m}, 2 \mathrm{H})$, 1.14-0.99 (m, 3H); ${ }^{13} \mathrm{C}$ NMR (126 MHz, $\left.\mathrm{CDCl}_{3}\right) \delta 144.3,141.4,135.7,135.3,130.5,129.7$, 128.6, 128.4, 124.9, 122.4, 121.4, 120.8, 119.1, 117.9, 108.8, 108.1, 33.7, 29.1, 23.4, 14.3; IR (KBr): 3411, 3367, 2965, 1705, 1346, $754 \mathrm{~cm}^{-1}$; HRMS (ESI): $\mathrm{m} / z$ calcd for $\mathrm{C}_{22} \mathrm{H}_{22} \mathrm{NO}$ $(\mathrm{M}+\mathrm{H})^{+}:$316.1701, found: 316.1694 .

\section{9-Methyl-4-phenyl-9H-carbazol-3-ol (3I):}

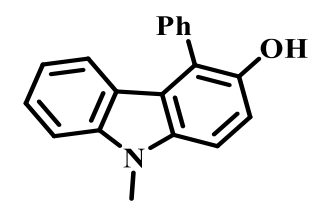

Following the general procedure $\mathrm{B}$, to the solution of 1-methyl- $1 H$-indole-2-carbaldehyde $1 \mathbf{a}$ (80 $\mathrm{mg}, 0.5 \mathrm{mmol})$ and 1-phenylprop-2-yn-1-ol $2 \mathrm{l}(66 \mathrm{mg}, 0.5 \mathrm{mmol})$ in $3 \mathrm{~mL}$ of dioxane, TfOH $(0.01 \mathrm{~mL}, 20 \mathrm{~mol} \%)$ was added at room temperature and stirred for $30 \mathrm{~min}$. Then, $\mathrm{Pd}\left(\mathrm{PPh}_{3}\right)_{4}(29$ $\mathrm{mg}, 5 \mathrm{~mol} \%$ ) was added to the reaction mixture and stirred at $80{ }^{\circ} \mathrm{C}$ for $7 \mathrm{~h}$. After completion of the reaction (monitored by TLC), the reaction mixture was concentrated under reduced pressure. The crude product was purified by column chromatography on silica gel (EtOAchexanes) to afford the product 9-methyl-4-phenyl-9H-carbazol-3-ol (3l), $100 \mathrm{mg}, 72 \%$ yield, off-white solid; $R_{f}=0.5$ (hexanes: EtOAc $\left.=9: 1\right)$; mp: $138-140{ }^{\circ} \mathrm{C} ;{ }^{1} \mathrm{H}$ NMR $(500 \mathrm{MHz}$, $\left.\mathrm{CDCl}_{3}\right) \delta 7.68-7.61(\mathrm{~m}, 2 \mathrm{H}), 7.61-7.55(\mathrm{~m}, 3 \mathrm{H}), 7.41-7.31(\mathrm{~m}, 3 \mathrm{H}), 7.23(\mathrm{~d}, J=8.7 \mathrm{~Hz}, 1 \mathrm{H})$, $7.01(\mathrm{~d}, J=8.0 \mathrm{~Hz}, 1 \mathrm{H}), 6.94-6.86(\mathrm{~m}, 1 \mathrm{H}), 4.84(\mathrm{~s}, 1 \mathrm{H}), 3.85(\mathrm{~s}, 3 \mathrm{H}) ;{ }^{13} \mathrm{C} \mathrm{NMR}(126 \mathrm{MHz}$, 
$\left.\mathrm{CDCl}_{3}\right) \delta 146.1,141.7,136.0,135.0,130.5,129.8,128.7,125.5,122.2,121.9,121.3,121.19$, 118.1, 114.2, 108.6, 108.3, 29.2; IR (KBr): 2987, 1733, 1372, 1240, 1046, $748 \mathrm{~cm}^{-1}$; HRMS (ESI): $m / z$ calcd for $\mathrm{C}_{19} \mathrm{H}_{16} \mathrm{NO}(\mathrm{M}+\mathrm{H})^{+}: 274.1232$, found: 274.1223 .

\section{4-(4-Methoxyphenyl)-9-methyl-9H-carbazol-3-ol (3m):}

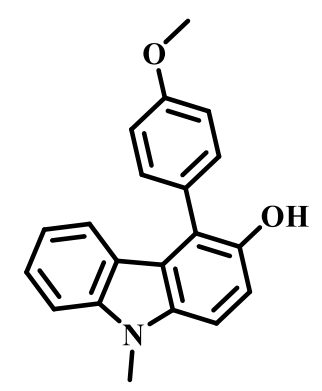

Following the general procedure $\mathrm{B}$, to the solution of 1-methyl- $1 H$-indole-2-carbaldehyde $1 \mathbf{a}$ (80 $\mathrm{mg}, 0.5 \mathrm{mmol})$ and 1-(4-methoxyphenyl)prop-2-yn-1-ol $2 \mathrm{~m}(81 \mathrm{mg}, 0.5 \mathrm{mmol})$ in $3 \mathrm{~mL}$ of dioxane, $\mathrm{TfOH}(0.01 \mathrm{~mL}, 20 \mathrm{~mol} \%)$ was added at room temperature and stirred for $30 \mathrm{~min}$. Then, $\mathrm{Pd}\left(\mathrm{PPh}_{3}\right)_{4}(29 \mathrm{mg}, 5 \mathrm{~mol} \%)$ was added and stirred at $80{ }^{\circ} \mathrm{C}$ for $7 \mathrm{~h}$. After completion of the reaction (monitored by TLC), the reaction mixture was concentrated under reduced pressure. The crude product was purified by column chromatography on silica gel (EtOAc-hexanes) to afford the product 4-(4-methoxyphenyl)-9-methyl-9H-carbazol-3-ol (3m), $120 \mathrm{mg}, 78 \%$ yield, off-white solid; $R_{f}=0.3$ (hexanes: EtOAc $\left.=9: 1\right)$; mp: $148-150{ }^{\circ} \mathrm{C} ;{ }^{1} \mathrm{H} \mathrm{NMR}(400 \mathrm{MHz}$, $\left.\mathrm{CDCl}_{3}\right) \delta 7.50-7.43(\mathrm{~m}, 2 \mathrm{H}), 7.40-7.28(\mathrm{~m}, 3 \mathrm{H}), 7.22-7.12(\mathrm{~m}, 3 \mathrm{H}), 7.05(\mathrm{~d}, J=7.9 \mathrm{~Hz}, 1 \mathrm{H})$, 6.94-6.83 (m, 1H), $4.83(\mathrm{~s}, 1 \mathrm{H}), 3.94(\mathrm{~s}, 3 \mathrm{H}), 3.84(\mathrm{~s}, 3 \mathrm{H}) ;{ }^{13} \mathrm{C} \mathrm{NMR}\left(101 \mathrm{MHz}, \mathrm{CDCl}_{3}\right) \delta$ 159.9, 146.4, 141.7, 135.9, 131.6, 126.7, 125.4, 122.3, 122.0, 121.5, 120.9, 118.1, 115.1, 114.0, 108.4, 108.3, 55.4, 29.1; IR (KBr): 3480, 3104, 2948, 2374, 1478, 1293, $802 \mathrm{~cm}^{-1}$; HRMS (ESI): $m / z$ calcd for $\mathrm{C}_{20} \mathrm{H}_{18} \mathrm{NO}_{2}(\mathrm{M}+\mathrm{H})^{+}: 304.1338$, found: 304.1353 .

\section{9-Methyl-4-(naphthalen-1-yl)-9H-carbazol-3-ol (3n):}

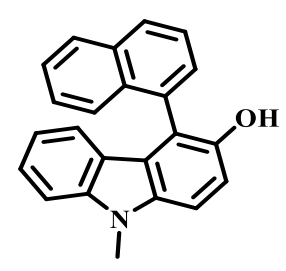

Following the general procedure B, to the solution of 1-methyl- $1 H$-indole-2-carbaldehyde 1a (80 $\mathrm{mg}, 0.5 \mathrm{mmol})$ and 1-(naphthalen-1-yl)prop-2-yn-1-ol $2 \mathrm{n}(91 \mathrm{mg}, 0.5 \mathrm{mmol})$ in $3 \mathrm{~mL}$ of dioxane, $\mathrm{TfOH}(0.01 \mathrm{~mL}, 20 \mathrm{~mol} \%)$ was added at room temperature and stirred for $30 \mathrm{~min}$. Then, $\mathrm{Pd}\left(\mathrm{PPh}_{3}\right)_{4}(29 \mathrm{mg}, 5 \mathrm{~mol} \%)$ was added to the reaction mixture and stirred at $80{ }^{\circ} \mathrm{C}$ for $7 \mathrm{~h}$. 
After completion of the reaction (monitored by TLC), the reaction mixture was concentrated under reduced pressure. The crude product was purified by column chromatography on silica gel (EtOAc-hexanes) to afford the product 9-methyl-4-(naphthalen-1-yl)-9H-carbazol-3-ol (3n), $115 \mathrm{mg}, 70 \%$ yield, off-white solid; $R_{f}=0.3$ (hexanes: EtOAc $=9: 1$ ); mp: $158-160{ }^{\circ} \mathrm{C}$; ${ }^{1} \mathrm{H}$ NMR $\left(400 \mathrm{MHz}, \mathrm{CDCl}_{3}\right) \delta 8.08(\mathrm{~d}, J=7.6 \mathrm{~Hz}, 1 \mathrm{H}), 8.00(\mathrm{~d}, J=8.2 \mathrm{~Hz}, 1 \mathrm{H}), 7.75-7.63$ (m, 2H), 7.58-7.48 (m, 2H), $7.43(\mathrm{~d}, J=8.7 \mathrm{~Hz}, 1 \mathrm{H}), 7.35-7.26(\mathrm{~m}, 4 \mathrm{H}), 6.72-6.62(\mathrm{~m}, 1 \mathrm{H})$, $6.45(\mathrm{~d}, J=7.9 \mathrm{~Hz}, 1 \mathrm{H}), 4.64(\mathrm{~s}, 1 \mathrm{H}), 3.89(\mathrm{~s}, 3 \mathrm{H}) ;{ }^{13} \mathrm{C} \mathrm{NMR}\left(101 \mathrm{MHz}, \mathrm{CDCl}_{3}\right) \delta 146.8$, 141.6, 136.0, 134.2, 132.3, 129.3, 128.6(2C), 128.5, 126.9, 126.6, 126.1, 125.7, 125.4, 122.1, 122.0, 121.8, 119.0, 118.1, 114.3, 109.0, 108.2, 29.8; IR (KBr): 3505, 3322, 2363, 2108, 1291, 1104, $816 \mathrm{~cm}^{-1}$; HRMS (ESI): $\mathrm{m} / z$ calcd for $\mathrm{C}_{23} \mathrm{H}_{18} \mathrm{NO}(\mathrm{M}+\mathrm{H})^{+}$: 324.1388 , found: 324.1377 .

\section{2,4-Diphenyl-9H-carbazol-3-ol (3o):}

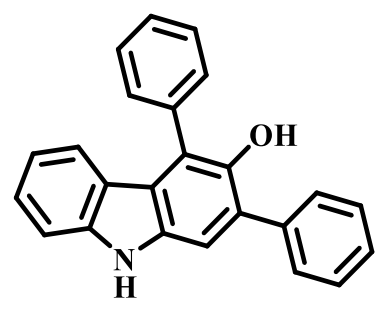

Following the general procedure $\mathrm{B}$, to the solution of $1 \mathrm{H}$-indole-2-carbaldehyde $\mathbf{1 b}$ ( $73 \mathrm{mg}, 0.50$ mmol) and 1,3-diphenylprop-2-yn-1-ol $2 \mathbf{a}(104 \mathrm{mg}, 0.5 \mathrm{mmol})$ in $3 \mathrm{~mL}$ of dioxane, $\mathrm{TfOH}(0.01$ $\mathrm{mL}, 20 \mathrm{~mol} \%)$ was added at room temperature and stirred for $30 \mathrm{~min}$. Then, $\mathrm{Pd}\left(\mathrm{PPh}_{3}\right)_{4}(29 \mathrm{mg}, 5$ mol \%) was added to the reaction mixture and stirred at $80{ }^{\circ} \mathrm{C}$ for $8 \mathrm{~h}$. After completion of the reaction (monitored by TLC), the reaction mixture was concentrated under reduced pressure. The crude product was purified by column chromatography on silica gel (EtOAchexanes) to afford the product 2,4-diphenyl-9H-carbazol-3-ol(3o), $115 \mathrm{mg}, 68 \%$ yield, offwhite solid; $R_{f}=0.3$ (hexanes: EtOAc $\left.=9: 1\right)$; mp: $195-197{ }^{\circ} \mathrm{C} ;{ }^{1} \mathrm{H}$ NMR $\left(500 \mathrm{MHz}, \mathrm{CDCl}_{3}\right) \delta$ 7.99 (s, 1H), 7.70-7.65 (m, 2H), 7.65-7.58 (m, 4H), 7.58-7.53 (m, 1H), 7.51-7.45 (m, 2H), 7.41-7.34 (m, 3H), 7.33-7.27 (m, 1H), $6.97(\mathrm{~d}, J=7.9 \mathrm{~Hz}, 1 \mathrm{H}), 6.92-6.86(\mathrm{~m}, 1 \mathrm{H}), 4.98(\mathrm{~s}$, $1 \mathrm{H}) ;{ }^{13} \mathrm{C}$ NMR $\left(126 \mathrm{MHz} \mathrm{CDCl}_{3}\right) \delta 143.4,140.7,138.7,135.4,134.2,130.5,129.7,129.6$, 128.6, 127.7, 127.3, 125.7 (2C), 123.0, 122.0, 121.8, 121.7, 118.9, 111.4, 110.5; IR (KBr): $3505,3322,2363,2108,1291,1104,816 \mathrm{~cm}^{-1}$; HRMS (ESI): $\mathrm{m} / \mathrm{z}$ calcd for $\mathrm{C}_{24} \mathrm{H}_{18} \mathrm{NO}$ $(\mathrm{M}+\mathrm{H})^{+}:$336.1388, found: 336.1380 .

\section{7,8,9-Trimethoxy-5-methyl-11-(phenylethynyl)-5H-benzo[b]carbazole (4a):}




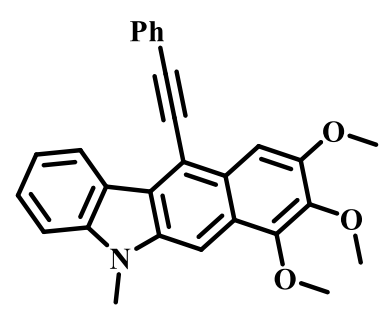

Following the general procedure B, to the solution of 1-methyl-1H-indole-2-carbaldehyde $1 \mathbf{a}$ (80 $\mathrm{mg}, 0.5 \mathrm{mmol}$ ) and 3-phenyl-1-(3,4,5-trimethoxyphenyl)prop-2-yn-1-ol 20 (149 mg, $0.5 \mathrm{mmol}$ ) in $3 \mathrm{~mL}$ of dioxane, $\mathrm{TfOH}(0.01 \mathrm{~mL}, 20 \mathrm{~mol} \%)$ was added at room temperature and stirred for 30 min. Then, $\mathrm{Pd}\left(\mathrm{PPh}_{3}\right)_{4}(29 \mathrm{mg}, 5 \mathrm{~mol} \%)$ was added to the reaction mixture and stirred at $80{ }^{\circ} \mathrm{C}$ for $4 \mathrm{~h}$. After completion of the reaction (monitored by TLC), the reaction mixture was concentrated under reduced pressure. The crude product was purified by column chromatography on silica gel (EtOAc-hexanes) to afford the product 7,8,9-trimethoxy-5methyl-11-(phenylethynyl)-5H-benzo[b]carbazole (4a), $180 \mathrm{mg}$, 85\% yield, off-white solid; $R_{f}=0.3$ (hexanes: EtOAc = 9:1); mp: $136-138{ }^{\circ} \mathrm{C} ;{ }^{1} \mathrm{H} \mathrm{NMR}\left(400 \mathrm{MHz}, \mathrm{CDCl}_{3}\right) \delta 8.90(\mathrm{~d}, J=$ $7.7 \mathrm{~Hz}, 1 \mathrm{H}), 7.94(\mathrm{~s}, 1 \mathrm{H}), 7.87-7.68(\mathrm{~m}, 3 \mathrm{H}), 7.60-7.52(\mathrm{~m}, 1 \mathrm{H}), 7.52-7.34(\mathrm{~m}, 4 \mathrm{H}), 7.33-$ $7.25(\mathrm{~m}, 1 \mathrm{H}), 4.16(\mathrm{~s}, 3 \mathrm{H}), 4.10(\mathrm{~s}, 3 \mathrm{H}), 4.05(\mathrm{~s}, 3 \mathrm{H}), 3.90(\mathrm{~s}, 3 \mathrm{H}) ;{ }^{13} \mathrm{C} \mathrm{NMR}(126 \mathrm{MHz}$, $\left.\mathrm{CDCl}_{3}\right) \delta 151.4,147.3,143.5,140.7,139.4,131.6,128.7,128.5,127.4,126.3,125.1,123.7$, $123.5,123.1,122.5,118.9,111.0,107.8,100.7,99.5,98.9,87.5,61.5,61.3,55.8,29.3$; IR (KBr): 3416, 2752, 2315, 1667, 1531, 1106, $723 \mathrm{~cm}^{-1}$; HRMS (ESI): $\mathrm{m} / \mathrm{z}$ calcd for $\mathrm{C}_{28} \mathrm{H}_{24} \mathrm{NO}_{3}(\mathrm{M}+\mathrm{H})^{+}:$422.1756, found: 422.1743 .

\section{9-Methyl-1,2,4-triphenyl-9H-carbazol-3-ol (5a):}

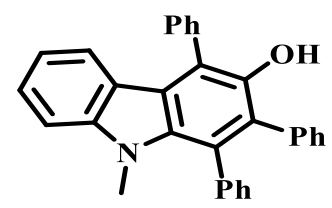

Following the general procedure $\mathrm{B}$, to the solution of (1-methyl-1H-indol-2-yl)(phenyl)methanone 1c (118 mg, $0.5 \mathrm{mmol})$ and 1,3-diphenylprop-2-yn-1-ol 2a (104 mg, $0.5 \mathrm{mmol})$ in $3 \mathrm{~mL}$ of dioxane, $\mathrm{TfOH}(0.01 \mathrm{~mL}, 20 \mathrm{~mol} \%)$ was added at room temperature and stirred for $30 \mathrm{~min}$. Then, $\mathrm{Pd}\left(\mathrm{PPh}_{3}\right)_{4}(29 \mathrm{mg}, 5 \mathrm{~mol} \%)$ was added to the reaction mixture and stirred at $80{ }^{\circ} \mathrm{C}$ for $6 \mathrm{~h}$. After completion of the reaction (monitored by TLC), the reaction mixture was concentrated under reduced pressure. The crude product was purified by column chromatography on silica gel (EtOAc-hexanes) to afford the product 9-methyl-1,2,4-triphenyl-9H-carbazol-3-ol (5a), $175 \mathrm{mg}, 82 \%$ yield, off-white solid; $R_{f}=0.3$ (hexanes: EtOAc $=9: 1$ ); mp: $245-247{ }^{\circ} \mathrm{C} ;{ }^{1} \mathrm{H}$ NMR (400 MHz, CDCl3) $\delta$ 7.68-7.57 (m, 4H), 7.57-7.49 (m, 1H), 7.38-7.30 (m, 1H), 7.28$7.13(\mathrm{~m}, 11 \mathrm{H}), 6.98(\mathrm{~d}, J=7.8 \mathrm{~Hz}, 1 \mathrm{H}), 6.91-6.83(\mathrm{~m}, 1 \mathrm{H}), 4.72(\mathrm{~s}, 1 \mathrm{H}), 3.17(\mathrm{~s}, 3 \mathrm{H}) ;{ }^{13} \mathrm{C}$ 
NMR (101 MHz, $\left.\mathrm{CDCl}_{3}\right) \delta 143.1,141.9,139.0,135.9,135.5,130.5(2 \mathrm{C}), 129.7$ (2C), 129.6(2C), 128.6(3C), 127.5, 127.4, 125.6, 122.3, 122.0, 121.9, 120.9, 118.3, 109.4, 108.3, 29.2; IR (KBr): 3542, 3055, 2929, 1725, 1390, 1157, $754 \mathrm{~cm}^{-1}$; HRMS (ESI): $\mathrm{m} / z$ calcd for $\mathrm{C}_{31} \mathrm{H}_{24} \mathrm{NO}(\mathrm{M}+\mathrm{H})^{+}: 426.1858$, found: 426.1858 .

\section{1-(4-Methoxyphenyl)-9-methyl-2,4-diphenyl-9H-carbazol-3-ol (5b):}

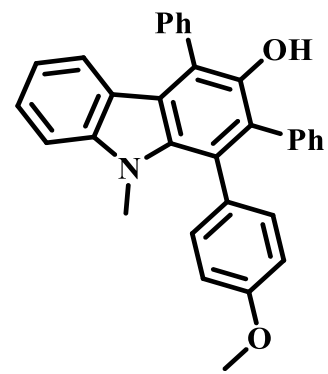

Following the general procedure $\mathrm{B}$, to the solution of (4-methoxyphenyl)(1-methyl-1H-indol-2yl)methanone 1d (135 mg, $0.5 \mathrm{mmol})$ and 1,3-diphenylprop-2-yn-1-ol 2a (105 mg, $0.5 \mathrm{mmol})$ in $3 \mathrm{~mL}$ of dioxane, $\mathrm{TfOH}(0.01 \mathrm{~mL}, 20 \mathrm{~mol} \%)$ was added at room temperature and stirred for 30 min. Then, $\mathrm{Pd}\left(\mathrm{PPh}_{3}\right)_{4}(29 \mathrm{mg}, 5 \mathrm{~mol} \%)$ was added to the reaction mixture and stirred at $80{ }^{\circ} \mathrm{C}$ for $6 \mathrm{~h}$. After completion of the reaction (monitored by TLC), the reaction mixture was concentrated under reduced pressure. The crude product was purified by column chromatography on silica gel (EtOAc-hexanes) to afford the product 1-(4-methoxyphenyl)-9methyl-2,4-diphenyl-9H-carbazol-3-ol (5b), $182 \mathrm{mg}$, 80\% yield, off-white solid; $R_{f}=0.3$ (hexanes:EtOAc = 9:1); mp: $225-227{ }^{\circ} \mathrm{C} ;{ }^{1} \mathrm{H}$ NMR (400 MHz, $\left.\mathrm{CDCl}_{3}\right) \delta 7.67-7.57$ (m, 4H), 7.57-7.50 (m, 1H), 7.37-7.29 (m, 1H), 7.28-7.23 (m, 3H), 7.22-7.12 (m, 5H), 6.97 (d, $J=$ $7.9 \mathrm{~Hz}, 1 \mathrm{H}), 6.92-6.82(\mathrm{~m}, 1 \mathrm{H}), 6.80-6.72(\mathrm{~m}, 2 \mathrm{H}), 4.70(\mathrm{~s}, 1 \mathrm{H}), 3.78(\mathrm{~s}, 3 \mathrm{H}), 3.20(\mathrm{~s}, 3 \mathrm{H})$; ${ }^{13} \mathrm{C} \mathrm{NMR}\left(101 \mathrm{MHz}, \mathrm{CDCl}_{3}\right) \delta 158.5,143.2,143.1,136.5,136.1,134.0,132.4,131.2,130.4$, $130.3,129.2$, 128.2, 128.0, 127.0, 125.5, 124.4, 122.3, 122.1, 121.7, 120.8, 118.3, 112.8, 108.6, 55.2, 32.4; IR (KBr): 3751, 3566, 3099, 1702, 1524, 1249, $760 \mathrm{~cm}^{-1}$; HRMS (ESI): $m / z$ calcd for $\mathrm{C}_{32} \mathrm{H}_{26} \mathrm{NO}_{2}(\mathrm{M}+\mathrm{H})^{+}:$456.1964, found: 456.1965 .

\section{9-Methyl-2,4-diphenyl-1-(4-(trifluoromethyl)phenyl)-9H-carbazol-3-ol (5c):}

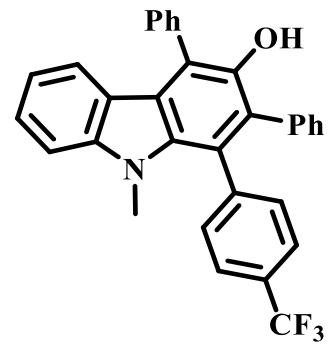

Following the general procedure $\mathrm{B}$, to the solution of (1-methyl-1H-indol-2-yl)(4(trifluoromethyl)phenyl)methanone 1e (150 mg, $0.5 \mathrm{mmol})$ and 1,3-diphenylprop-2-yn-1-ol $\mathbf{2 a}$ 
(103 mg, $0.5 \mathrm{mmol})$ in $3 \mathrm{~mL}$ of dioxane, TfOH $(0.01 \mathrm{~mL}, 20 \mathrm{~mol} \%)$ was added at room temperature and stirred for $30 \mathrm{~min}$. Then, $\mathrm{Pd}\left(\mathrm{PPh}_{3}\right)_{4}(29 \mathrm{mg}, 5 \mathrm{~mol} \%)$ was added to the reaction mixture and stirred at $80{ }^{\circ} \mathrm{C}$ for $6 \mathrm{~h}$. After completion of the reaction (monitored by TLC), the reaction mixture was concentrated under reduced pressure. The crude product was purified by column chromatography on silica gel (EtOAc-hexanes) to afford the product 9methyl-2,4-diphenyl-1-(4-(trifluoromethyl)phenyl)-9H-carbazol-3-ol (5c), 185 mg, $75 \%$ yield, yellow solid; $\mathrm{R}_{f}=0.3$ (hexanes: EtOAc $\left.=9: 1\right)$; mp: $258-260{ }^{\circ} \mathrm{C} ;{ }^{1} \mathrm{H}$ NMR $(300 \mathrm{MHz}$, CDCl3) $\delta$ 7.68-7.46 (m, 7H), 7.45-7.31 (m, 3H), $7.27(\mathrm{~s}, 1 \mathrm{H}), 7.25-7.12(\mathrm{~m}, 5 \mathrm{H}), 6.97(\mathrm{~d}, J$ $=7.7 \mathrm{~Hz}, 1 \mathrm{H}), 6.89(\mathrm{t}, J=7.4 \mathrm{~Hz}, 1 \mathrm{H}), 4.74(\mathrm{~s}, 1 \mathrm{H}), 3.16(\mathrm{~s}, 3 \mathrm{H}) ;{ }^{13} \mathrm{C}$ NMR $(101 \mathrm{MHz}$, $\left.\mathrm{CDCl}_{3}\right) \delta 143.3,143.1,142.2,135.8,135.8,133.4,131.8,131.1,130.3,129.3,129.2(\mathrm{q}, J=$ $32.5 \mathrm{~Hz}), 129.0,128.4,128.3,127.5,127.4,125.9,125.5,124.5$ (q, $J=272.2 \mathrm{~Hz}), 124.3$, 124.3, 123.0, 122.8, 122.2, 121.6, 118.7, 108.7, 32.8; IR (KBr): 3688, 3021, 2404, 1530, 1418, 1215, $740 \mathrm{~cm}^{-1}$; HRMS (ESI): $\mathrm{m} / z$ calcd for $\mathrm{C}_{32} \mathrm{H}_{23} \mathrm{NOF}_{3}(\mathrm{M}+\mathrm{H})^{+}:$494.1732, found: 494.1734.

\section{9-Methyl-2,4-diphenyl-1-(thiophen-3-yl)-9H-carbazol-3-ol (5d):}

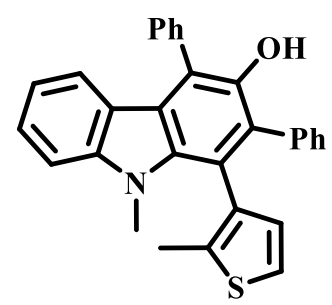

Following the general procedure B, to the solution of (1-methyl-1H-indol-2-yl)(2-methylthiophen3-yl)methanone 1f (130 mg, $0.5 \mathrm{mmol})$ and 1,3-diphenylprop-2-yn-1-ol 2a (103 mg, $0.5 \mathrm{mmol})$ in $3 \mathrm{~mL}$ of dioxane, $\mathrm{TfOH}(0.01 \mathrm{~mL}, 20 \mathrm{~mol} \%)$ was added at room temperature and stirred for 30 min. Then, $\mathrm{Pd}\left(\mathrm{PPh}_{3}\right)_{4}(29 \mathrm{mg}, 5 \mathrm{~mol} \%)$ was added to the reaction mixture and stirred at $80{ }^{\circ} \mathrm{C}$ for $6 \mathrm{~h}$. After completion of the reaction (monitored by TLC) the reaction mixture was concentrated under reduced pressure. The crude product was purified by column chromatography on silica gel (EtOAc-hexanes) to afford the product 9-methyl-2,4-diphenyl1-(thiophen-3-yl)-9H-carbazol-3-ol (5d), $155 \mathrm{mg}, 70 \%$ yield, yellow solid; $R_{f}=0.3$ (hexanes: EtOAc = 9:1); mp: $189-191{ }^{\circ} \mathrm{C} ;{ }^{1} \mathrm{H}$ NMR $(500 \mathrm{MHz}, \mathrm{CDCl} 3) \delta$ 7.65-7.52 (m, 5H), 7.41-7.25 $(\mathrm{m}, 7 \mathrm{H}), 7.17(\mathrm{~d}, J=5.1 \mathrm{~Hz}, 1 \mathrm{H}), 6.96(\mathrm{~d}, J=7.9 \mathrm{~Hz}, 1 \mathrm{H}), 6.88(\mathrm{t}, J=7.4 \mathrm{~Hz}, 1 \mathrm{H}), 6.75(\mathrm{~d}, J$ $=5.1 \mathrm{~Hz}, 1 \mathrm{H}), 4.69(\mathrm{~s}, 1 \mathrm{H}), 3.33(\mathrm{~s}, 3 \mathrm{H}), 1.99(\mathrm{~s}, 3 \mathrm{H}) ;{ }^{13} \mathrm{C} \mathrm{NMR}\left(101 \mathrm{MHz}, \mathrm{CDCl}_{3}\right) \delta 143.2$, $143.0,137.1,136.1,135.9,134.7,133.2,131.0,130.3,130.2,129.5,129.2,129.3,128.7$, $128.2,128.1,127.4,125.7,125.3,122.1,121.8,118.4,115.6,108.6,30.5,14.7$; IR (KBr): 
3689, 2928, 2101, 1539, 1458, 1216, $757 \mathrm{~cm}^{-1}$; HRMS (ESI): $\mathrm{m} / z$ calcd for $\mathrm{C}_{30} \mathrm{H}_{24} \mathrm{NOS}$ $(\mathrm{M}+\mathrm{H})^{+}:$446.1579, found: 446.1573 .

\section{1,9-Dimethyl-2,4-diphenyl-9H-carbazol-3-ol (5e):}

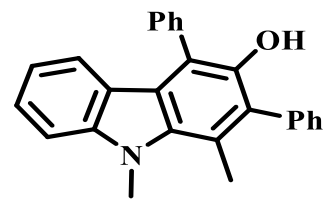

Following the general procedure $\mathrm{B}$, to the solution of 1-(1-methyl- $1 H$-indol-2-yl)ethanone $1 \mathrm{~g}$ (88 $\mathrm{mg}, 0.5 \mathrm{mmol})$ and 1,3-diphenylprop-2-yn-1-ol 2a (103 $\mathrm{mg}, 0.5 \mathrm{mmol})$ in $3 \mathrm{~mL}$ of dioxane, $\mathrm{TfOH}(0.01 \mathrm{~mL}, 20 \mathrm{~mol} \%)$ was added at room temperature and stirred for $30 \mathrm{~min}$. Then, $\mathrm{Pd}\left(\mathrm{PPh}_{3}\right)_{4}(29 \mathrm{mg}, 5 \mathrm{~mol} \%)$ was added to the reaction mixture and stirred at $80{ }^{\circ} \mathrm{C}$ for $6 \mathrm{~h}$. After completion of the reaction (monitored by TLC), the reaction mixture was concentrated under reduced pressure. The crude product was purified by column chromatography on silica gel (EtOAc-hexanes) to afford the product 1,9-dimethyl-2,4-diphenyl-9H-carbazol-3-ol (5e), $137 \mathrm{mg}, 75 \%$ yield, brown solid; $\mathrm{R}_{f}=0.3$ (hexanes: EtOAc $=9: 1$ ); mp: $174-176{ }^{\circ} \mathrm{C} ;{ }^{1} \mathrm{H} \mathrm{NMR}$ $\left(400 \mathrm{MHz}, \mathrm{CDCl}_{3}\right) \delta$ 7.64-7.61 (m, 1H), 7.61-7.58 (m, 3H), 7.57-7.53 (m, 3H), 7.49-7.38 (m, 4H), 7.38-7.36 (m, 1H), 6.98-6.93 (m, 1H), 6.92-6.87 (m, 1H), $4.64(\mathrm{~s}, 1 \mathrm{H}), 4.13$ (s, 3H), $2.63(\mathrm{~s}, 3 \mathrm{H}) ;{ }^{13} \mathrm{C} \mathrm{NMR}\left(126 \mathrm{MHz}, \mathrm{CDCl}_{3}\right) \delta 143.6,143.1,137.3,136.0,135.2,130.8$, $130.5,129.3,128.9$, 128.6, 128.2, 127.7, 125.5, 122.6, 122.1, 121.6, 119.3, 119.0, 118.3, 108.6, 33.3, 17.5; IR (KBr): 3553, 3017, 2967, 1551, 1181, 1030, $754 \mathrm{~cm}^{-1}$; HRMS (ESI): $m / z$ calcd for $\mathrm{C}_{26} \mathrm{H}_{22} \mathrm{NO}(\mathrm{M}+\mathrm{H})^{+}: 364.1701$, found: 364.1700 .

\section{9-Methyl-2,4-diphenyl-1-propyl-9H-carbazol-3-ol (5f):}

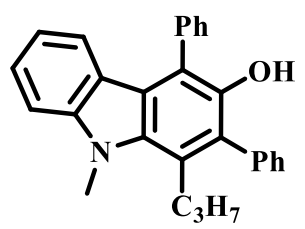

Following the general procedure $\mathrm{B}$, to the solution of 1-(1-methyl-1H-indol-2-yl)butan-1-one $\mathbf{1 h}$ (100 mg, $0.5 \mathrm{mmol}$ ) and 1,3-diphenylprop-2-yn-1-ol 2a (103 mg, $0.5 \mathrm{mmol})$ in $3 \mathrm{~mL}$ of dioxane, $\mathrm{TfOH}(0.01 \mathrm{~mL}, 20 \mathrm{~mol} \%)$ was added at room temperature and stirred for $30 \mathrm{~min}$. Then, $\mathrm{Pd}\left(\mathrm{PPh}_{3}\right)_{4}(29 \mathrm{mg}, 5 \mathrm{~mol} \%)$ was added to the reaction mixture and stirred at $80{ }^{\circ} \mathrm{C}$ for $6 \mathrm{~h}$. After completion of the reaction (monitored by TLC), the reaction mixture was concentrated under reduced pressure. The crude product was purified by column chromatography on silica gel (EtOAc-hexanes) to afford the product 9-methyl-2,4-diphenyl-1-propyl-9H-carbazol-3-ol 
(5f), $128 \mathrm{mg}, 65 \%$ yield, off-white solid; $\mathrm{R}_{f}=0.3$ (hexanes: EtOAc = 9:1); mp: $199-201{ }^{\circ} \mathrm{C}$; ${ }^{1} \mathrm{H}$ NMR $\left(500 \mathrm{MHz}, \mathrm{CDCl}_{3}\right) \delta 7.60-7.47(\mathrm{~m}, 7 \mathrm{H}), 7.47-7.38(\mathrm{~m}, 3 \mathrm{H}), 7.35(\mathrm{~d}, J=3.3 \mathrm{~Hz}$, 2H), $6.89(\mathrm{~d}, J=7.9 \mathrm{~Hz}, 1 \mathrm{H}), 6.87-6.81(\mathrm{~m}, 1 \mathrm{H}), 4.50(\mathrm{~s}, 1 \mathrm{H}), 4.06(\mathrm{~s}, 3 \mathrm{H}), 2.98-2.78(\mathrm{~m}$, $2 \mathrm{H}), 1.71-1.55(\mathrm{~m}, 2 \mathrm{H}), 0.85(\mathrm{t}, J=7.3 \mathrm{~Hz}, 3 \mathrm{H}) ;{ }^{13} \mathrm{C} \mathrm{NMR}\left(126 \mathrm{MHz}, \mathrm{CDCl}_{3}\right) \delta 143.6$, 143.1, 137.2, 136.1, 134.2, 130.7, 130.5, 129.2, 128.8 (2C), 128.4, 128.1, 127.7, 125.4, 124.3, 122.7, 122.1, 119.3, 118.3, 108.5, 32.4, 31.5, 25.5, 14.1; IR (KBr): 3322, 2944, 2830, 1723, 1582, 1026, $757 \mathrm{~cm}^{-1}$; HRMS (ESI): $\mathrm{m} / z$ calcd for $\mathrm{C}_{28} \mathrm{H}_{26} \mathrm{NO}(\mathrm{M}+\mathrm{H})^{+}$: 392.2014, found: 392.2008 .

1-Cyclopropyl-9-methyl-2,4-diphenyl-9H-carbazol-3-ol (5g):

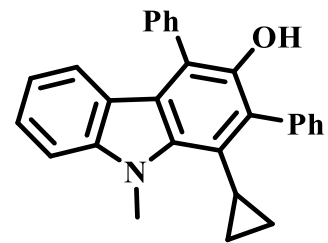

Following the general procedure $\mathrm{B}$, to the solution of cyclopropyl(1-methyl-1H-indol-2yl)methanone $1 \mathbf{i}$ (100 mg, $0.5 \mathrm{mmol}$ ) and 1,3-diphenylprop-2-yn-1-ol $\mathbf{2 a}$ (103 mg, $0.5 \mathrm{mmol})$ in 3 $\mathrm{mL}$ of dioxane, $\mathrm{TfOH}(0.01 \mathrm{~mL}, 20 \mathrm{~mol} \%)$ was added at room temperature and stirred for $30 \mathrm{~min}$. Then, $\mathrm{Pd}\left(\mathrm{PPh}_{3}\right)_{4}(29 \mathrm{mg}, 5 \mathrm{~mol} \%)$ was added to the reaction mixture and stirred at $80{ }^{\circ} \mathrm{C}$ for $6 \mathrm{~h}$. After completion of the reaction (monitored by TLC), the reaction mixture was concentrated under reduced pressure. The crude product was purified by column chromatography on silica gel (EtOAc-hexanes) to afford the product 1-cyclopropyl-9methyl-2,4-diphenyl-9H-carbazol-3-ol (5g), $150 \mathrm{mg}$, 77\% yield, yellow solid; $\mathrm{R}_{f}=0.3$ (hexanes: EtOAc = 9:1); mp: $239-241{ }^{\circ} \mathrm{C} ;{ }^{1} \mathrm{H}$ NMR $\left(500 \mathrm{MHz}, \mathrm{CDCl}_{3}\right) \delta 7.60-7.51(\mathrm{~m}, 5 \mathrm{H})$, 7.51-7.46 (m, 4H), 7.41-7.37 (m, 1H), 7.37-7.34 (m, 2H), 6.90-6.83 (m, 2H), $4.78(\mathrm{~s}, 1 \mathrm{H})$, $4.24(\mathrm{~s}, 3 \mathrm{H}), 2.30-2.19(\mathrm{~m}, 1 \mathrm{H}), 0.79-0.70(\mathrm{~m}, 2 \mathrm{H}), 0.54-0.44(\mathrm{~m}, 2 \mathrm{H}) ;{ }^{13} \mathrm{C} \mathrm{NMR}(101 \mathrm{MHz}$, $\left.\mathrm{CDCl}_{3}\right) \delta 143.9,143.3,137.9,137.3,136.1,131.1,130.4,130.0,129.2,128.5,128.1,127.2$, $125.5,123.8,122.9,122.0,121.9,120.2$, 118.5, 109.0, 34.0, 13.4, 11.2; IR (KBr): 3688, 3020, 2404, 1532, 1419, 1215, $740 \mathrm{~cm}^{-1}$; HRMS (ESI): $\mathrm{m} / z$ calcd for $\mathrm{C}_{28} \mathrm{H}_{24} \mathrm{NO}(\mathrm{M}+\mathrm{H})^{+}$: 390.1858, found: 390.1854 .

1-Cyclopentyl-9-methyl-2,4-diphenyl-9H-carbazol-3-ol (5h): 


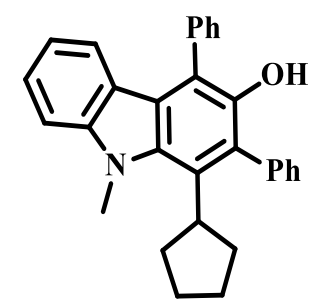

Following the general procedure B, cyclopentyl(1-methyl- $1 H$-indol-2-yl)methanone $\mathbf{1 j}$ (115 mg, $0.5 \mathrm{mmol})$ and 1,3-diphenylprop-2-yn-1-ol $\mathbf{2 a}(103 \mathrm{mg}, 0.5 \mathrm{mmol})$ in $3 \mathrm{~mL}$ of dioxane $\mathrm{TfOH}$ $(0.01 \mathrm{~mL}, 20 \mathrm{~mol} \%)$ was added at room temperature and stirred for $30 \mathrm{~min}$. Then, $\mathrm{Pd}\left(\mathrm{PPh}_{3}\right)_{4}(29$ $\mathrm{mg}, 5 \mathrm{~mol} \%$ ) was added to the reaction mixture and stirred at $80{ }^{\circ} \mathrm{C}$ for $6 \mathrm{~h}$. After completion of the reaction (monitored by TLC), the reaction mixture was concentrated under reduced pressure. The crude product was purified by column chromatography on silica gel (EtOAchexanes) to afford the product 1-cyclopentyl-9-methyl-2,4-diphenyl-9H-carbazol-3-ol (5h), $145 \mathrm{mg}, 70 \%$ yield, off-white solid; $R_{f}=0.3$ (hexanes: EtOAc $=9: 1$ ); mp: $182-184{ }^{\circ} \mathrm{C} ;{ }^{1} \mathrm{H}$ NMR (500 MHz, $\left.\mathrm{CDCl}_{3}\right) \delta 7.71(\mathrm{~d}, J=8.1 \mathrm{~Hz}, 1 \mathrm{H}), 7.59-7.53(\mathrm{~m}, 2 \mathrm{H}), 7.47-7.41(\mathrm{~m}, 2 \mathrm{H})$, 7.38-7.32 (m, 2H), 7.32-7.27 (m, 5H), 7.25-7.19 (m, 1H), 7.11-7.05 (m, 1H), $5.91(\mathrm{~s}, 1 \mathrm{H})$, $3.84(\mathrm{~s}, 3 \mathrm{H}), 3.72-3.60(\mathrm{~m}, 1 \mathrm{H}), 2.00-1.90(\mathrm{~m}, 1 \mathrm{H}), 1.90-1.80(\mathrm{~m}, 1 \mathrm{H}), 1.80-1.67(\mathrm{~m}, 4 \mathrm{H})$, $1.63-1.57(\mathrm{~m}, 1 \mathrm{H}), 1.55-1.49(\mathrm{~m}, 1 \mathrm{H}) ;{ }^{13} \mathrm{C} \mathrm{NMR}\left(101 \mathrm{MHz}, \mathrm{CDCl}_{3}\right) \delta 144.5,144.3,137.7$, 136.4 , 136.2, 131.5, 130.4, 129.2, 128.6, 128.0, 127.8, 127.0, 125.6, 123.2, 123.0, 122.8, 122.1, 119.5, 118.7, 109.3, 41.6, 35.8, 33.8, 26.1; IR (KBr): 3546, 3015, 1608, 1469, 1387, 1145, $755 \mathrm{~cm}^{-1}$; HRMS (ESI): $\mathrm{m} / z$ calcd for $\mathrm{C}_{30} \mathrm{H}_{28} \mathrm{NO}(\mathrm{M}+\mathrm{H})^{+}: 418.2171$, found: 418.2172 .

\section{1,9-Dimethyl-2-phenyl-4-(3,4,5-trimethoxyphenyl)-9H-carbazol-3-ol (5i):}

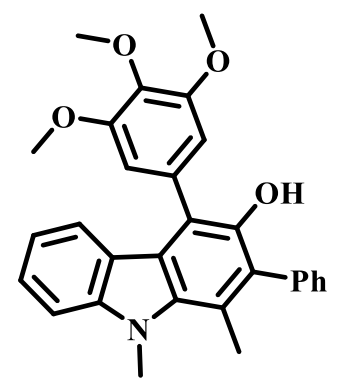

Following the general procedure $\mathrm{B}$, to the solution of 1-(1-methyl- $1 H$-indol-2-yl)ethanone $1 \mathrm{~g}$ (88 $\mathrm{mg}, 0.5 \mathrm{mmol}$ )and 1,3-diphenylprop-2-yn-1-ol $\mathbf{2 a}(103 \mathrm{mg}, 0.5 \mathrm{mmol})$ in $3 \mathrm{ml}$ of dioxane TfOH $(0.01 \mathrm{ml}, 20 \mathrm{~mol} \%)$ was added at room temperature and stirred for $30 \mathrm{~min}$. Then, $\mathrm{Pd}\left(\mathrm{PPh}_{3}\right)_{4}(29$ $\mathrm{mg}, 5 \mathrm{~mol} \%$ ) was added to the reaction mixture and stirred at $80{ }^{\circ} \mathrm{C}$ for $6 \mathrm{~h}$. After completion of the reaction (monitored by TLC), the reaction mixture was concentrated under reduced pressure. The crude product was purified by column chromatography on silica gel (EtOAc- 
hexanes) to afford the product 1,9-dimethyl-2-phenyl-4-(3,4,5-trimethoxyphenyl)-9Hcarbazol-3-ol (5i), $177 \mathrm{mg}, 78 \%$ yield, off-white solid; $R_{f}=0.3$ (hexanes: EtOAc $=9: 1$ ); mp: 220-222 ${ }^{\circ} \mathrm{C} ;{ }^{1} \mathrm{H}$ NMR (400 MHz, $\left.\mathrm{CDCl}_{3}\right) \delta$ 7.57-7.50 (m, 2H), 7.47-7.33 (m, 5H), $7.06(\mathrm{~d}, J$ $=7.9 \mathrm{~Hz}, 1 \mathrm{H}), 6.97-6.88(\mathrm{~m}, 1 \mathrm{H}), 6.78(\mathrm{~s}, 2 \mathrm{H}), 4.76(\mathrm{~s}, 1 \mathrm{H}), 4.13(\mathrm{~s}, 3 \mathrm{H}), 3.99(\mathrm{~s}, 3 \mathrm{H}), 3.84$ (s, 6H), $2.61(\mathrm{~s}, 3 \mathrm{H}) ;{ }^{13} \mathrm{C} \mathrm{NMR}\left(126 \mathrm{MHz}, \mathrm{CDCl}_{3}\right) \delta 153.0,142.6,142.1,136.7,136.3,134.1$, 130.1, 129.7 (2C), 127.8 (2C), 127.6, 126.6, 124.5, 121.4, 121.2, 120.5, 118.2, 117.9, 117.5, 107.6, 106.1, 60.1, 55.1 (2C), 32.2, 16.4; IR (KBr): 3094, 2921, 1682, 1599, 1460, 1105, 708 $\mathrm{cm}^{-1}$; MS (ESI): HRMS (ESI): $m / z$ calcd for $\mathrm{C}_{29} \mathrm{H}_{28} \mathrm{NO}_{4}(\mathrm{M}+\mathrm{H})^{+}: 454.2018$, found: 454.2020 .

10-(4-Methoxyphenyl)-5-methyl-2-phenyl-5H-furo[3,2-b]carbazole (7a):

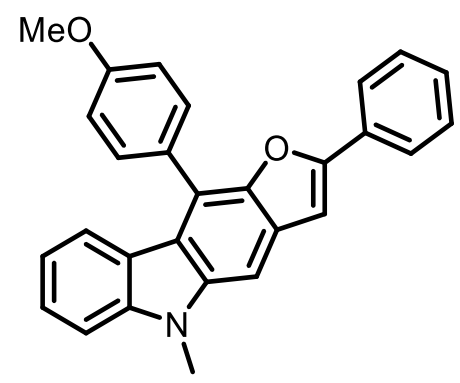

Following the general procedure $\mathrm{B}$, to the solution of 1-methyl- $1 H$-indole-2-carbaldehyde $1 \mathbf{a}$ (80 $\mathrm{mg}, 0.5 \mathrm{mmol}$ ) and 1-(4-methoxyphenyl)-5-phenylpenta-2,4-diyn-1-ol 6a (132 mg, $0.5 \mathrm{mmol})$ in $3 \mathrm{~mL}$ of dioxane, $\mathrm{TfOH}(0.01 \mathrm{~mL}, 20 \mathrm{~mol} \%)$ was added at room temperature and stirred for 30 min. Then, $\mathrm{Pd}\left(\mathrm{PPh}_{3}\right)_{4}(29 \mathrm{mg}, 5 \mathrm{~mol} \%)$ was added to the reaction mixture and stirred at $80{ }^{\circ} \mathrm{C}$ for $8 \mathrm{~h}$. After completion of the reaction (monitored by TLC), the reaction mixture was concentrated under reduced pressure. The crude product was purified by column chromatography on silica gel (EtOAc-hexanes) to afford the product 10-(4-methoxyphenyl)5-methyl-2-phenyl-5H-furo[3,2-b]carbazole (7a), $120 \mathrm{mg}$, 59\% yield, brown semi solid; $\mathrm{R}_{f}=$ 0.3 (hexanes: EtOAc = 9:1); ${ }^{1} \mathrm{H}$ NMR $\left(400 \mathrm{MHz}, \mathrm{CDCl}_{3}\right) \delta 7.82(\mathrm{~d}, J=7.7 \mathrm{~Hz}, 2 \mathrm{H}), 7.74(\mathrm{~d}$, $J=8.3 \mathrm{~Hz}, 2 \mathrm{H}), 7.56(\mathrm{~d}, J=7.9 \mathrm{~Hz}, 1 \mathrm{H}), 7.48-7.36(\mathrm{~m}, 5 \mathrm{H}), 7.36-7.29(\mathrm{~m}, 1 \mathrm{H}), 7.21-7.14$ $(\mathrm{m}, 3 \mathrm{H}), 6.97(\mathrm{t}, J=7.4 \mathrm{~Hz}, 1 \mathrm{H}), 3.99(\mathrm{~s}, 3 \mathrm{H}), 3.90(\mathrm{~s}, 3 \mathrm{H}) ;{ }^{13} \mathrm{C} \mathrm{NMR}\left(101 \mathrm{MHz}, \mathrm{CDCl}_{3}\right) \delta$ $159.9,159.4,156.8,142.3,139.5,136.7,131.5,130.7,128.7,128.4,127.7,127.3,125.4$, 125.0, 122.9, 122.1, 119.5, 118.0, 114.0, 107.9, 101.5, 97.0, 55.4, 29.3; IR (KBr): 3056, 2942, 1636, 1262, 943, $733 \mathrm{~cm}^{-1}$; HRMS (ESI): $\mathrm{m} / z$ calcd for $\mathrm{C}_{28} \mathrm{H}_{22} \mathrm{NO}_{2}(\mathrm{M}+\mathrm{H})^{+}: 404.1651$, found: 404.1646 .

2-Hexyl-5-methyl-10-(naphthalen-1-yl)-5H-furo[3,2-b]carbazole (7b): 


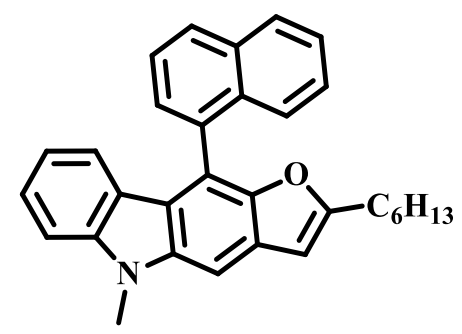

Following the general procedure $\mathrm{B}$, to the solution of 1-methyl- $1 H$-indole-2-carbaldehyde $1 \mathbf{a}$ (80 $\mathrm{mg}, 0.5 \mathrm{mmol})$ and 1-(naphthalen-1-yl)non-2-yn-1-ol 6c (134 mg, $0.5 \mathrm{mmol})$ in $3 \mathrm{~mL}$ of dioxane, $\mathrm{TfOH}(0.01 \mathrm{~mL}, 20 \mathrm{~mol} \%)$ was added at room temperature and stirred for $30 \mathrm{~min}$. Then, $\mathrm{Pd}\left(\mathrm{PPh}_{3}\right)_{4}(29 \mathrm{mg}, 5 \mathrm{~mol} \%)$ was added to the reaction mixture and stirred at $80{ }^{\circ} \mathrm{C}$ for $8 \mathrm{~h}$. After the completion of the reaction (monitored by TLC), the reaction mixture was concentrated under reduced pressure. The crude product was purified by column chromatography on silica gel (EtOAc-hexanes) to afford the product 2-hexyl-5-methyl-10(naphthalen-1-yl)-5H-furo[3,2-b]carbazole $(7 \mathbf{b}), 110 \mathrm{mg}, 51 \%$ yield, yellow semi solid; $\mathrm{R}_{f}=$ 0.3 (hexanes: EtOAc = 9:1); ${ }^{1} \mathrm{H}$ NMR $\left(300 \mathrm{MHz}, \mathrm{CDCl}_{3}\right) \delta 8.13-7.95(\mathrm{~m}, 2 \mathrm{H}), 7.76-7.63(\mathrm{~m}$, 2H), 7.53-7.42 (m, 3H), 7.37-7.27 (m, 2H), 7.24-7.17 (m, 1H), 6.76-6.66 (m, 1H), 6.66$6.50(\mathrm{~m}, 2 \mathrm{H}), 3.92(\mathrm{~s}, 3 \mathrm{H}), 2.66(\mathrm{t}, J=7.5 \mathrm{~Hz}, 2 \mathrm{H}), 1.71-1.58(\mathrm{~m}, 2 \mathrm{H}), 1.38-1.24(\mathrm{~m}, 6 \mathrm{H})$, $0.84(\mathrm{t}, J=6.5 \mathrm{~Hz}, 3 \mathrm{H}) ;{ }^{13} \mathrm{C} \mathrm{NMR}\left(101 \mathrm{MHz}, \mathrm{CDCl}_{3}\right) \delta 161.5,148.4,142.0,138.8,133.8$, $133.3,132.3,128.4,128.3,128.2$, 128.0, 126.3, 126.1, 125.9, 125.7, 124.9, 122.8, 121.9, 119.0, 118.0, 117.0, 107.7, 101.8, 97.0, 31.5, 29.3, 28.9, 28.7, 27.6, 22.6, 14.1; IR (neat): 3651, 3278, 2363, 1703, 1050, $799 \mathrm{~cm}^{-1}$; HRMS (ESI): $\mathrm{m} / z$ calcd for $\mathrm{C}_{31} \mathrm{H}_{30} \mathrm{NO}(\mathrm{M}+\mathrm{H})^{+}$: 432.2327, found: 432.2328 .

\section{Gram scale reaction procedure for 3a:}

To a stirred solution of 1-methyl- $1 H$-indole-2-carbaldehyde $1 \mathrm{a}(1 \mathrm{~g}, 6.28 \mathrm{mmol})$ and propargylic alcohol 2a $(1.31 \mathrm{~g}, 6.28 \mathrm{mmol})$ in $30 \mathrm{~mL}$ of dioxane in $\mathrm{RBF}$ was added $\mathrm{TfOH}$ $(0.11 \mathrm{~mL}, 20 \mathrm{~mol} \%)$ at room temperature and stirred for $30 \mathrm{~min}$. Then, $\mathrm{Pd}\left(\mathrm{PPh}_{3}\right)_{4}(363 \mathrm{mg}, 5$ mol \%) was added and stirred at $80{ }^{\circ} \mathrm{C}$ (oil bath temperature) for $8 \mathrm{~h}$. After completion of the reaction (monitored by TLC) the reaction mixture was concentrated under reduced pressure. The crude product was purified by column chromatography on silica gel by using EtOAc: hexanes as an eluant to afford the 9-methyl-2,4-diphenyl-9H-carbazol-3-ol $(\mathbf{3 a}), R_{f}=0.4$ (hexanes: EtOAc = 9:1), $1.71 \mathrm{~g}, 78 \%$ yield. 


\section{Crystal structure determination of 3a:}

X-ray Crystallography data for the compound KA860 was collected at room temperature on a Bruker D8 QUEST instrument with an $\mathrm{I} \mu \mathrm{S}$ Mo microsource $(\lambda=0.7107 \mathrm{~A})$ and a PHOTON-100 detector. The raw data frames were reduced and corrected for absorption effects using the Bruker Apex 3 software suite programs. ${ }^{2}$ The structure was solved using intrinsic phasing method ${ }^{3}$ and further refined with the SHELXL ${ }^{3}$ program and expanded using Fourier techniques. Anisotropic displacement parameters were included for all nonhydrogen atoms. The O-bound $\mathrm{H}$ atom was located in difference Fourier maps and their positions and isotropic displacement parameters were located and refined.All $\mathrm{C}$ bound $\mathrm{H}$ atoms were positioned geometrically and treated as riding on their parent $\mathrm{C}$ atoms $[\mathrm{C}-\mathrm{H}=$ 0.93-0.97 $\AA$ and $\mathrm{U}_{\text {iso }}(\mathrm{H})=1.5 \mathrm{U}_{\mathrm{eq}}(\mathrm{C})$ for methyl $\mathrm{H}$ or $1.2 \mathrm{U}_{\mathrm{eq}}(\mathrm{C})$ for other $\mathrm{H}$ atoms].

Crystal Datafor3a, $\mathrm{C}_{25} \mathrm{H}_{19} \mathrm{NO}(M=349.41 \mathrm{~g} / \mathrm{mol})$ : monoclinic, space group $\mathrm{P} 2{ }_{1} / \mathrm{c}$ (no. 14), $a=14.1248(4) \AA, \quad b=12.0055(3) \AA, \quad c=11.2593(3) \AA, \quad \beta=93.6930(10)^{\circ}, \quad V=$ 1905.33(9) $\AA^{3}, Z=4, T=294.15 \mathrm{~K}, \mu(\mathrm{MoK} \alpha)=0.074 \mathrm{~mm}^{-1}$, Dcalc $=1.218 \mathrm{~g} / \mathrm{cm}^{3}, 20326$ reflections measured $\left(4.456^{\circ} \leq 2 \Theta \leq 49.99^{\circ}\right), 3356$ unique $\left(R_{\text {int }}=0.0518, \mathrm{R}_{\text {sigma }}=0.0358\right)$ which were used in all calculations. The final $R_{1}$ was $0.0715(\mathrm{I}>2 \sigma(\mathrm{I}))$ and $w R_{2}$ was 0.2121 (all data). CCDC Number 1971748 contains supplementary Crystallographic data for the structure. These data can be obtained free of charge at www.ccdc.cam.ac.uk/conts/retrieving.html [or from the Cambridge Crystallographic Data Centre (CCDC), 12 Union Road, Cambridge CB2 1EZ, UK; fax: +44(0) 1223336 033; email: deposit@ccdc.cam.ac.uk].

\section{References:}

1.(a) Liu, P.; Deng, C.-L.; Lei, X.; Lin, G.-q. Eur. J. Org. Chem. 2011, 7308. (b) Egi, M.; Kawai, T.; Umemura, M.; Akai, S. J. Org. Chem. 2012, 77, 7092. (c) Ugajin, R.; Kikuchi, S.; Yamada, T. Synlett 2014, 25, 1178. (d) Wilson, E. E.; Oliver, A. G.; Hughes R. P.; Ashfeld, B. L. Organometallics, 2011, 30, 5214. (e) G.-C. Ge, D.-L. Mo, C.-H. Ding, L.-X. Dai, X.-L. Hou, A. Org. Lett. 2012, 14, 5756. (f) Reddy, C. R.; Vijaykumar, J.; Grée, R. Synthesis 2013, 45, 830. (g) Chen, J.; Fan, G.; Liu, Y. Org. Biomol. Chem. 2010, 8, 4806. (h) Reddy, C. R.; Aila, M.; Sathish, P.; Mrinalini, M.; Giribabu, L.; Prasanthkumar, S.; Grée, R. Org. Biomol. Chem. 2019, 17, 9291. 
2. Bruker (2001). SAINT (Version 6.28a) \&SMART (Version 5.625). Bruker AXS Inc., Madison, Wisconsin, USA.

3. Sheldrick G. M. (2015) Acta Crystallogr C71: 3-8. 
6. ${ }^{1} \mathrm{H}$ NMR and ${ }^{13} \mathrm{C}$ NMR Spectral Copies of compounds:
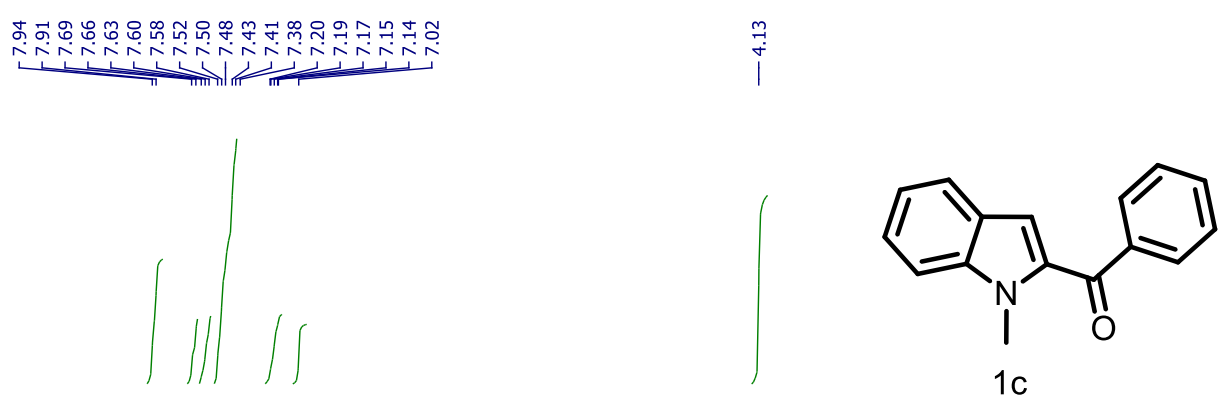

${ }^{1} \mathrm{H} \mathrm{NMR}, 300 \mathrm{MHz}, \mathrm{CDCl}_{3}$

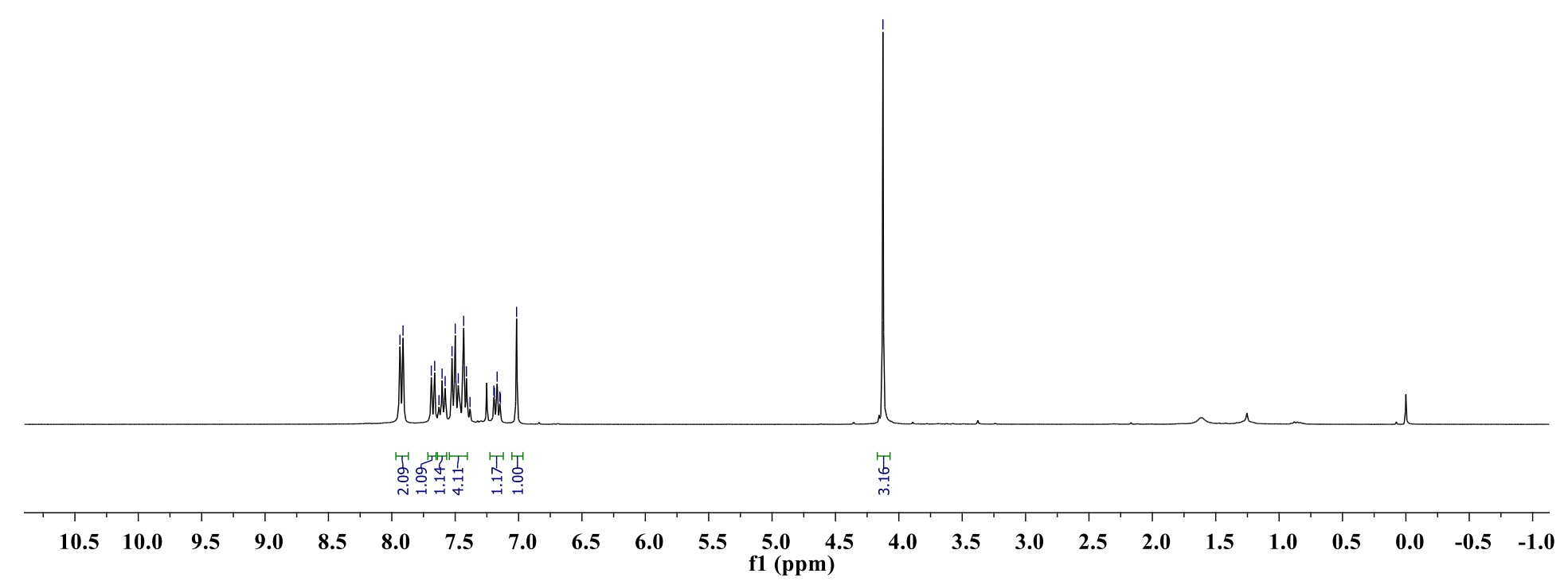



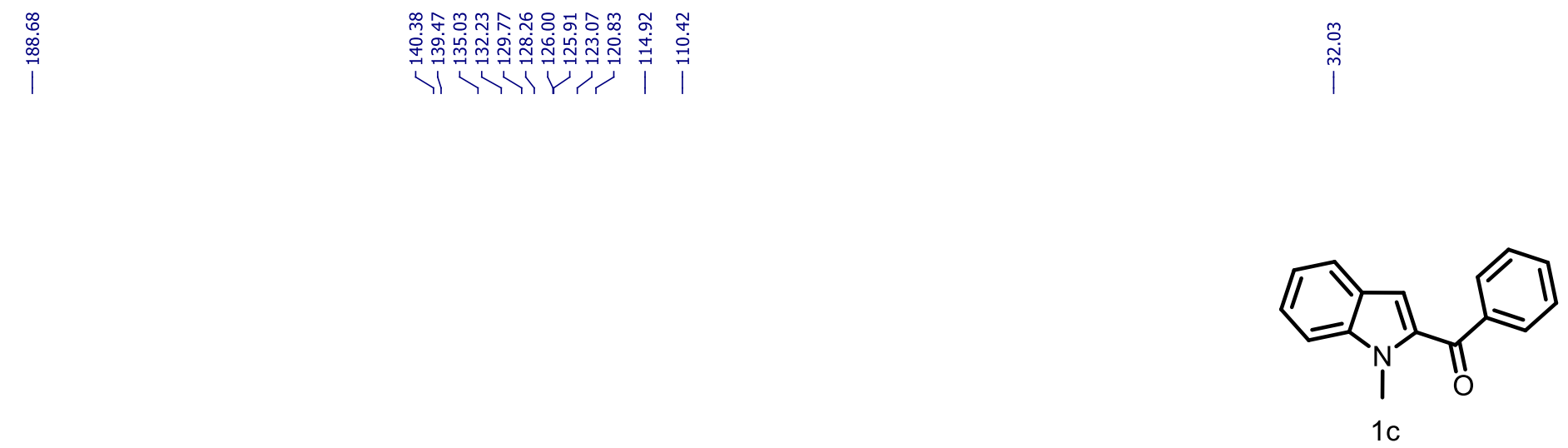

${ }^{13} \mathrm{C}$ NMR, $101 \mathrm{MHz}, \mathrm{CDCl}_{3}$
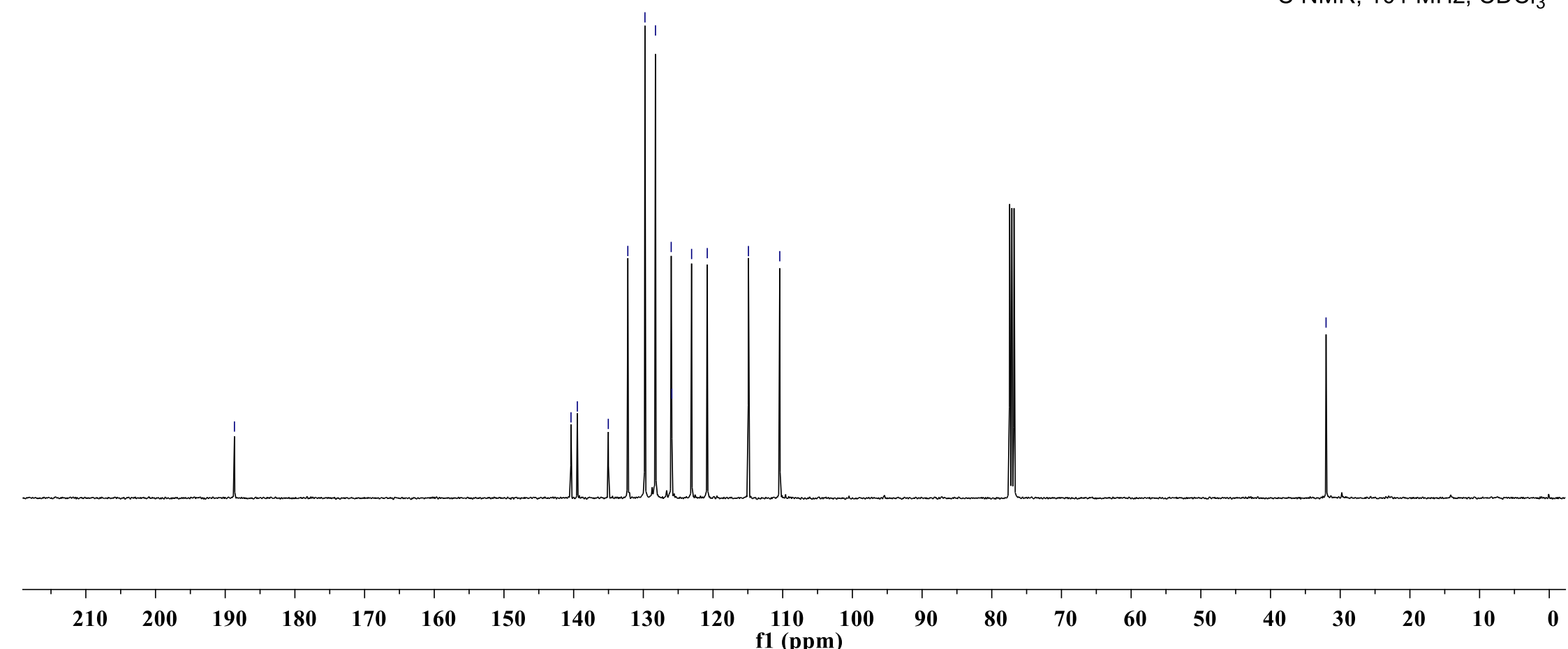


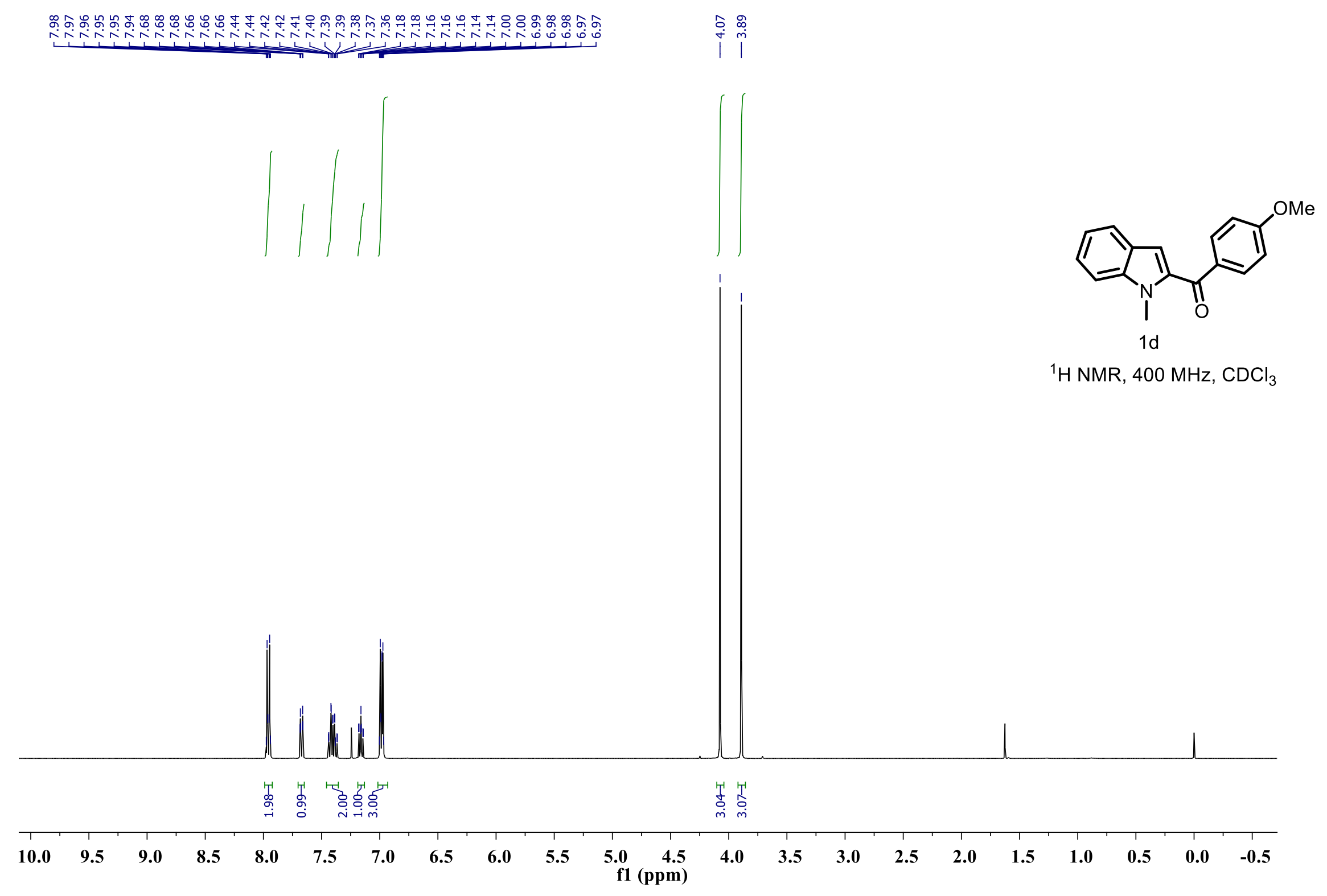




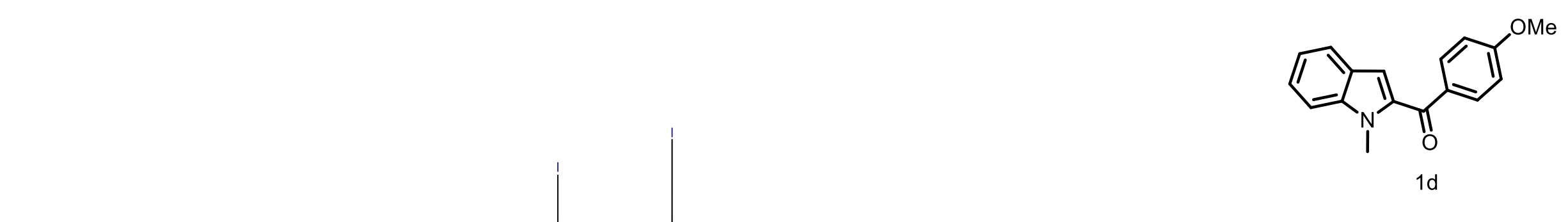

${ }^{13} \mathrm{C} N M R, 101 \mathrm{MHz}, \mathrm{CDCl}_{3}$

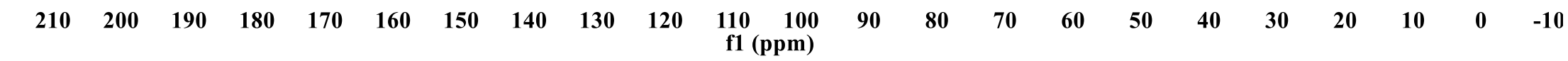




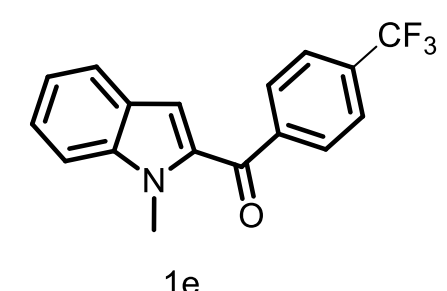

${ }^{1} \mathrm{H} \mathrm{NMR}, 400 \mathrm{MHz}, \mathrm{CDCl}_{3}$

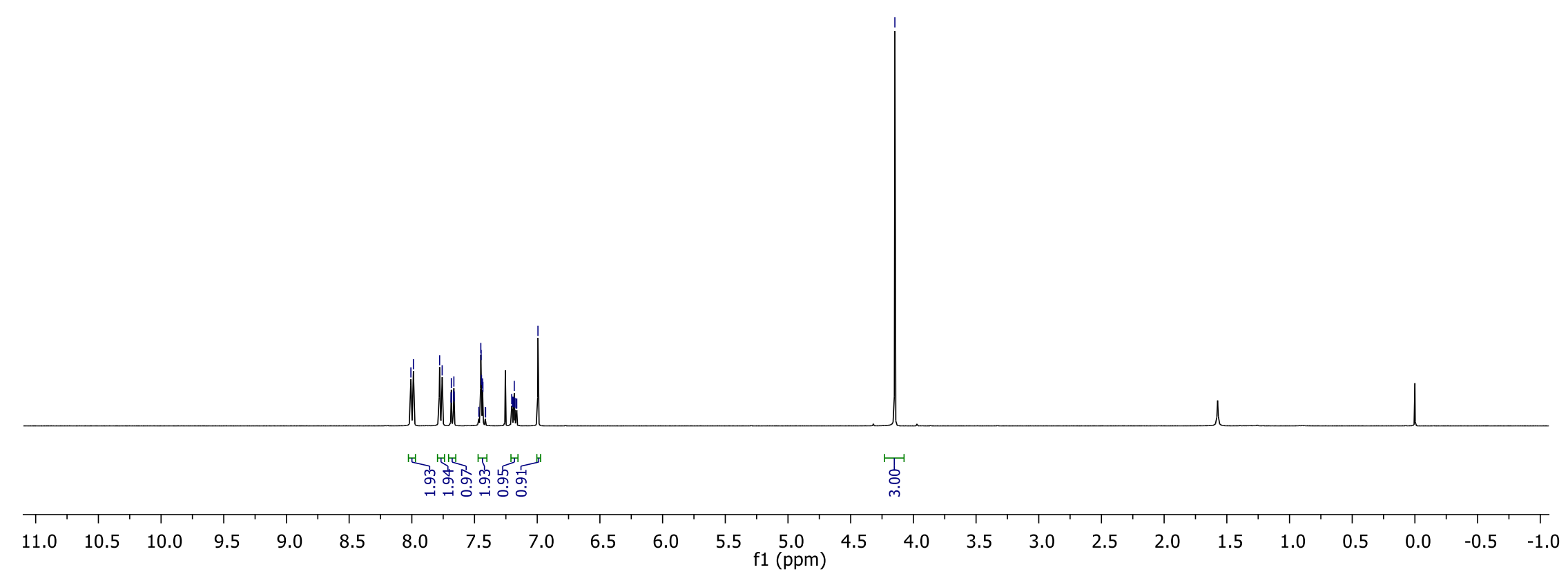



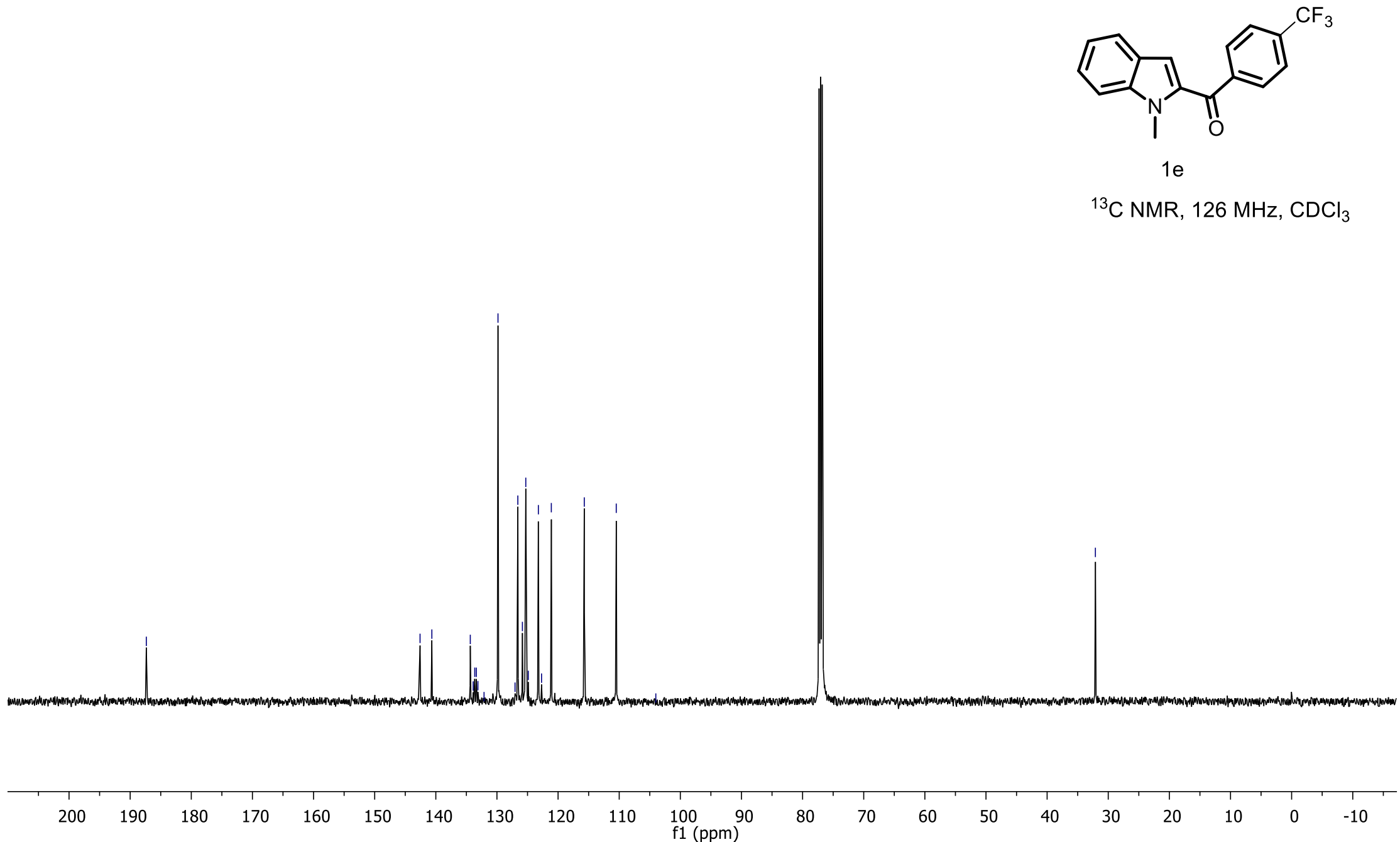


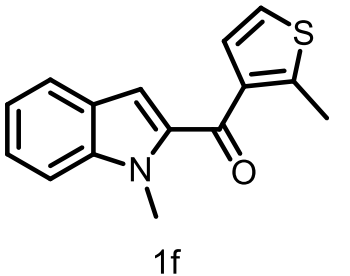

${ }^{1} \mathrm{H}$ NMR, $400 \mathrm{MHz}, \mathrm{CDCl}_{3}$ )

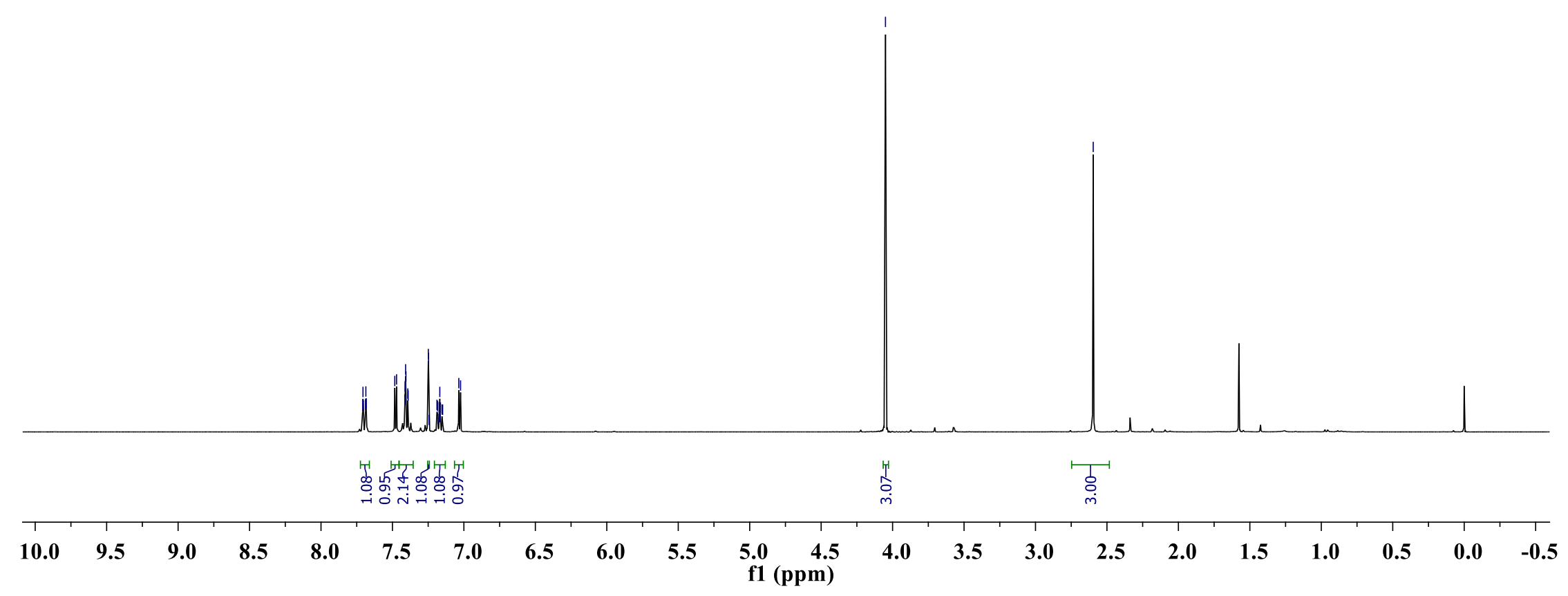




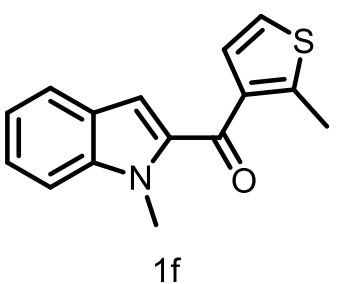

${ }^{13} \mathrm{C} \mathrm{NMR}, 101 \mathrm{MHz}, \mathrm{CDCl}_{3}$

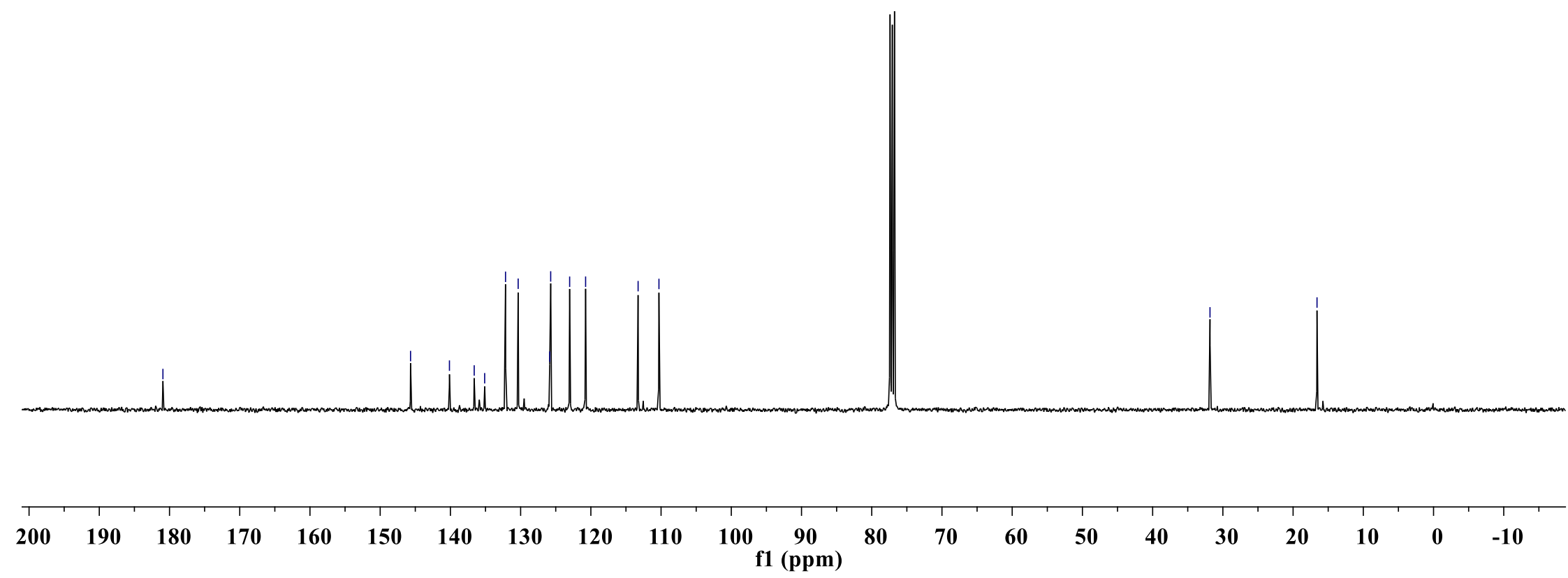



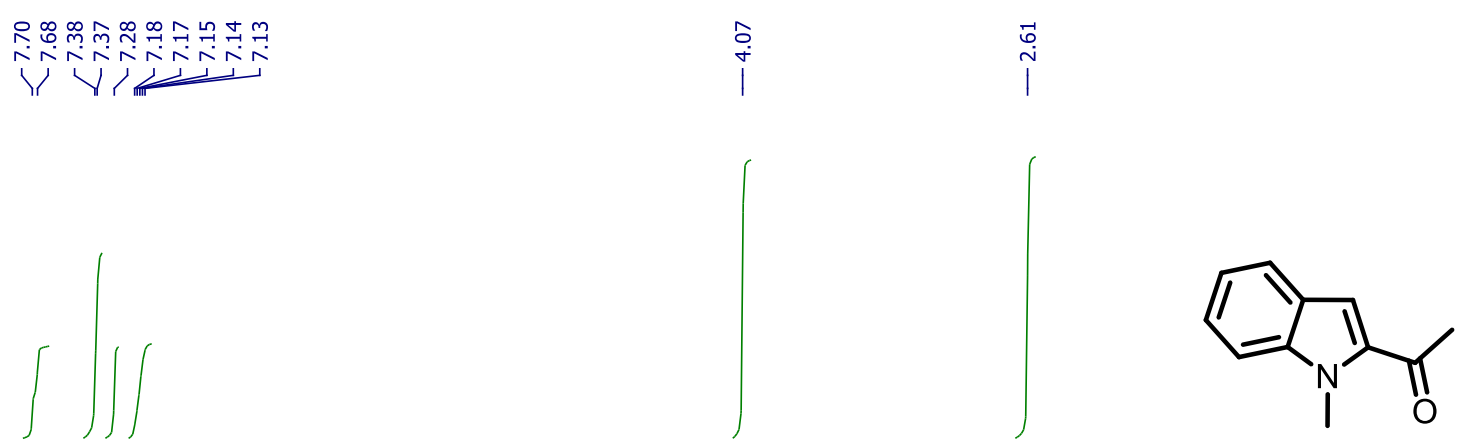

$1 \mathrm{~g}$

${ }^{1} \mathrm{H}$ NMR, $300 \mathrm{MHz}, \mathrm{CDCl}_{3}$

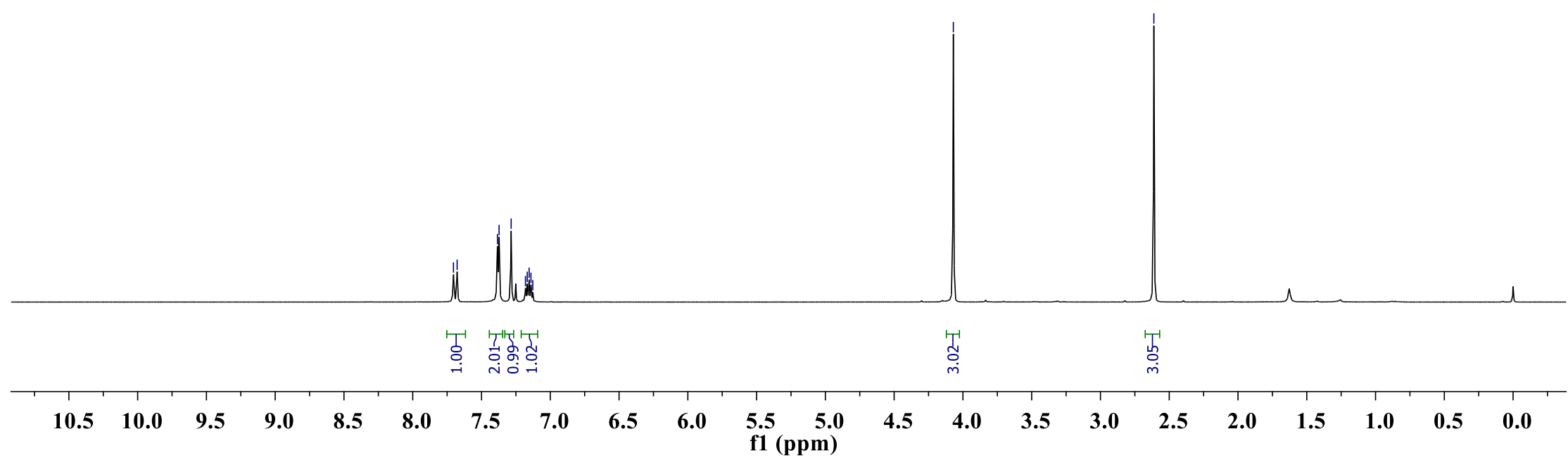



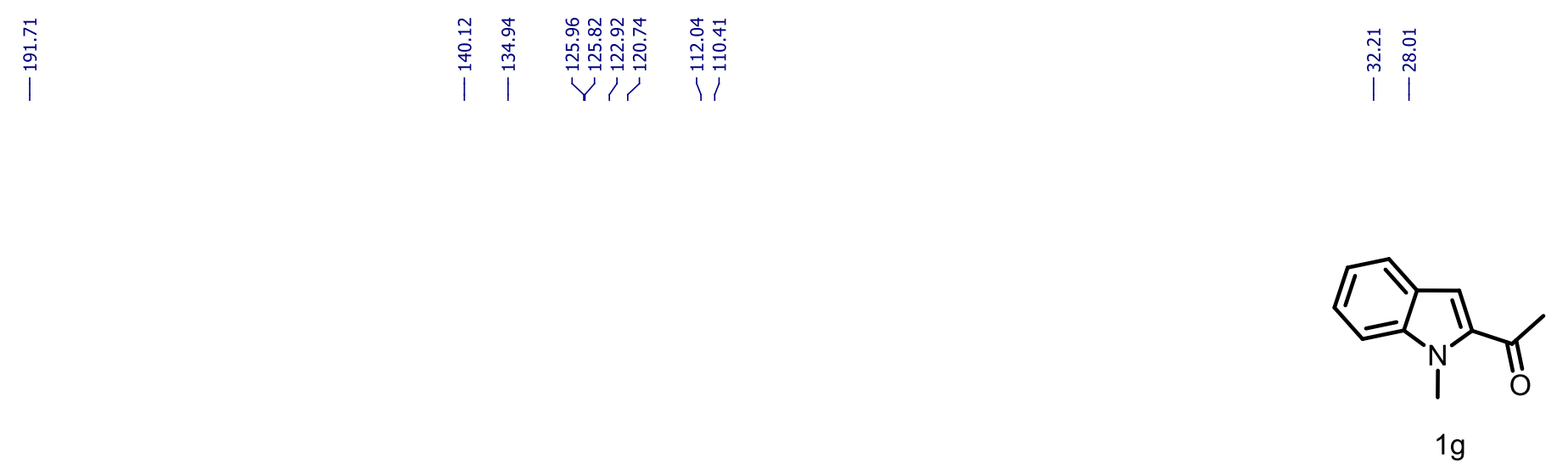

${ }^{13} \mathrm{C}$ NMR, $101 \mathrm{MHz}, \mathrm{CDCl}_{3}$
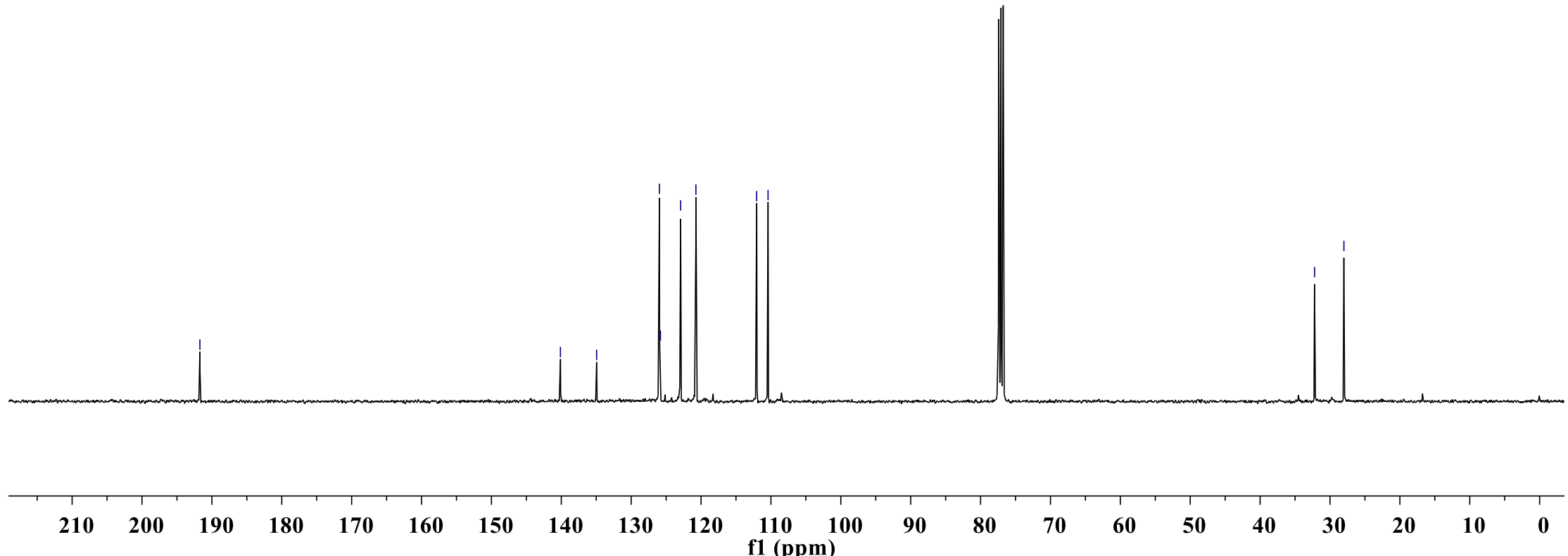


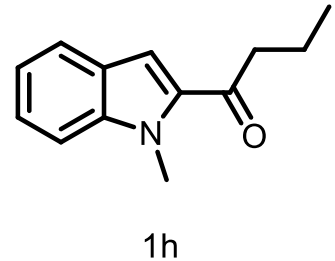

${ }^{1} \mathrm{H} \mathrm{NMR}, 300 \mathrm{MHz}, \mathrm{CDCl}_{3}$

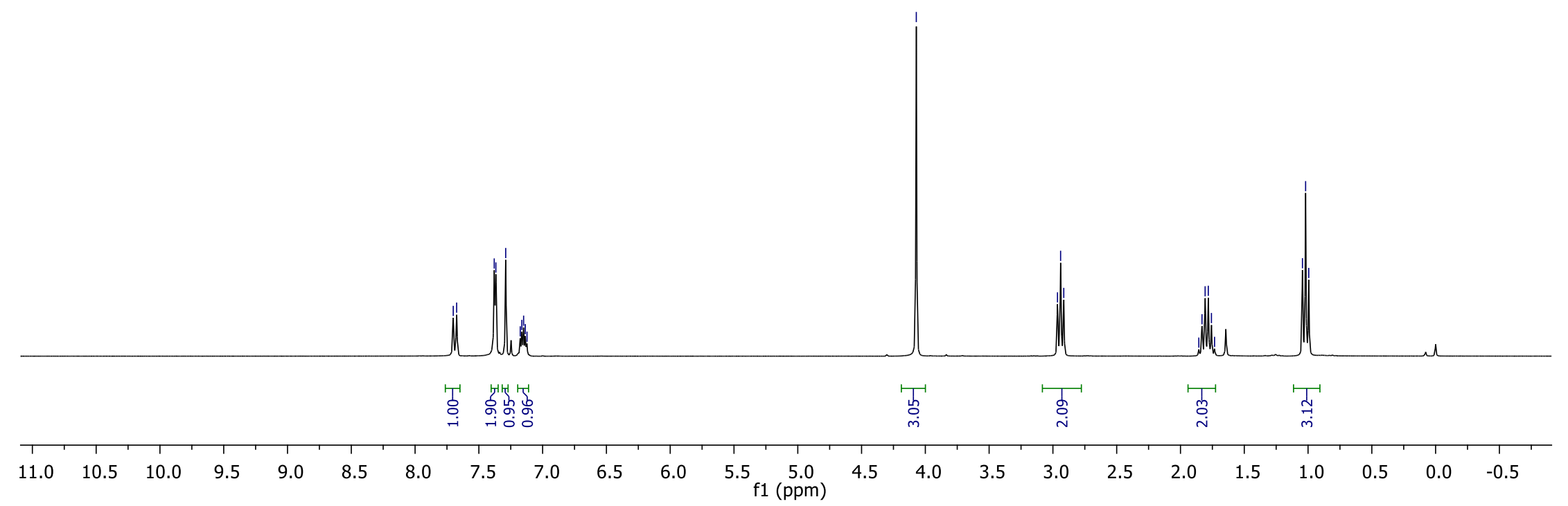




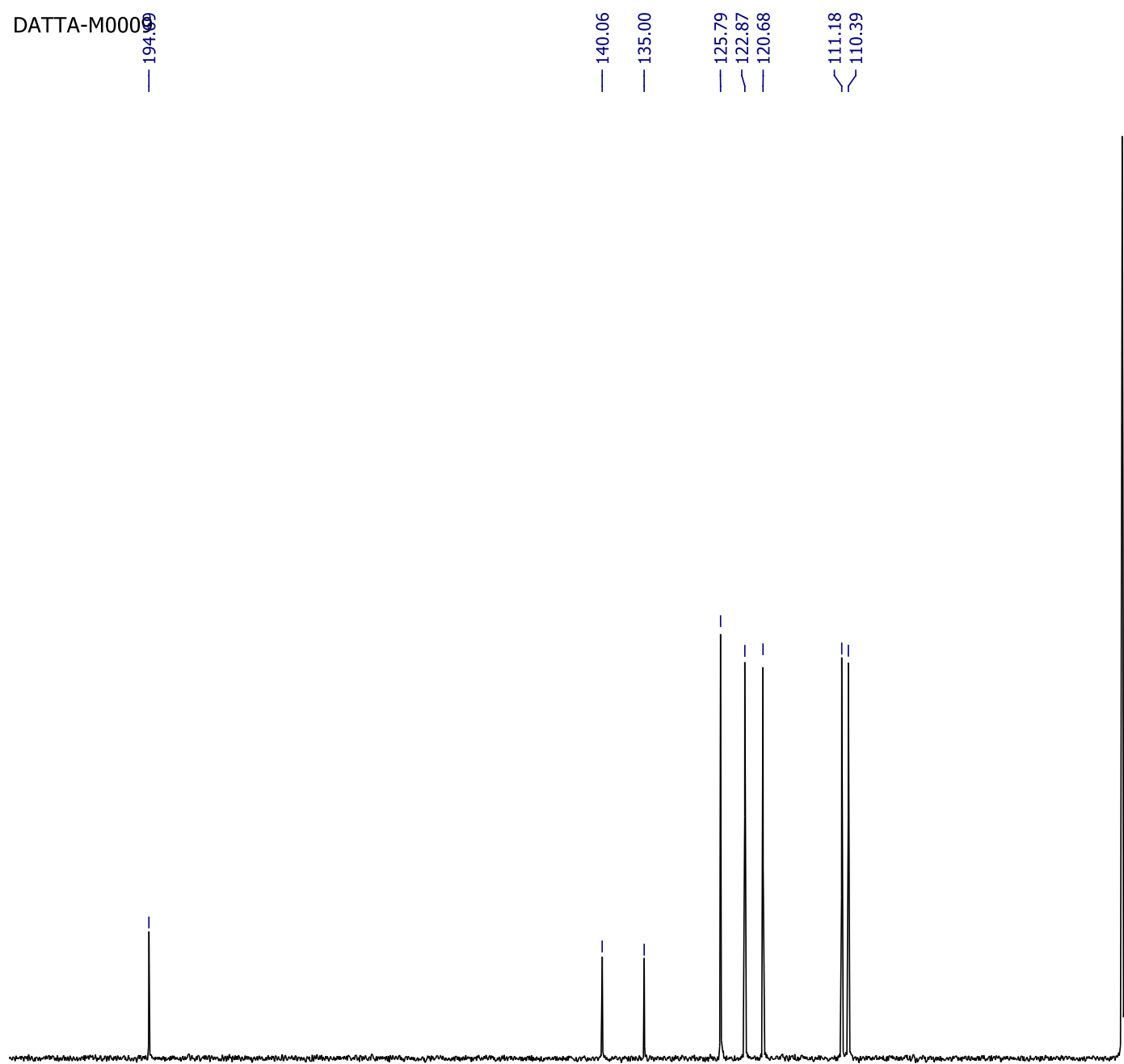

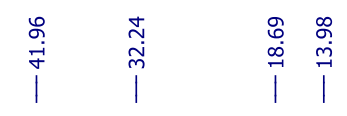

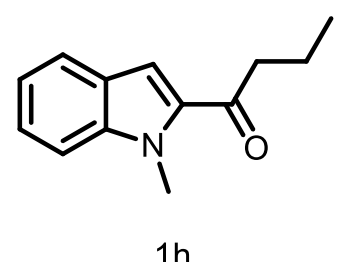

${ }^{13} \mathrm{C}$ NMR, $101 \mathrm{MHz}, \mathrm{CDCl}_{3}$

170

160

140

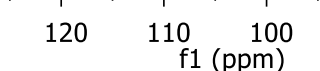

f1 $(\mathrm{ppm})$ 

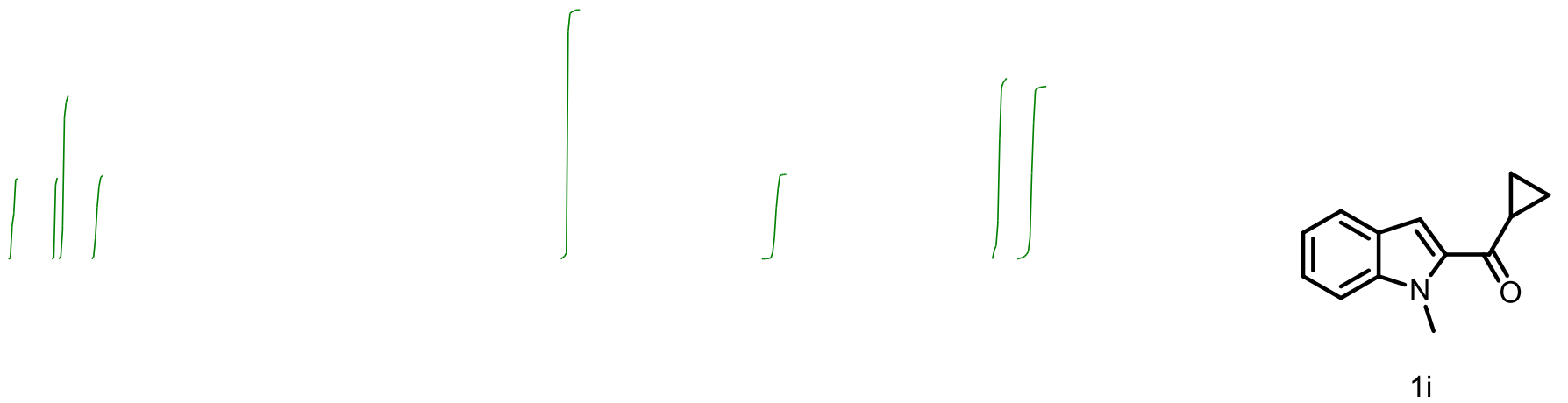

${ }^{1} \mathrm{H}$ NMR, $500 \mathrm{MHz}, \mathrm{CDCl}_{3}$

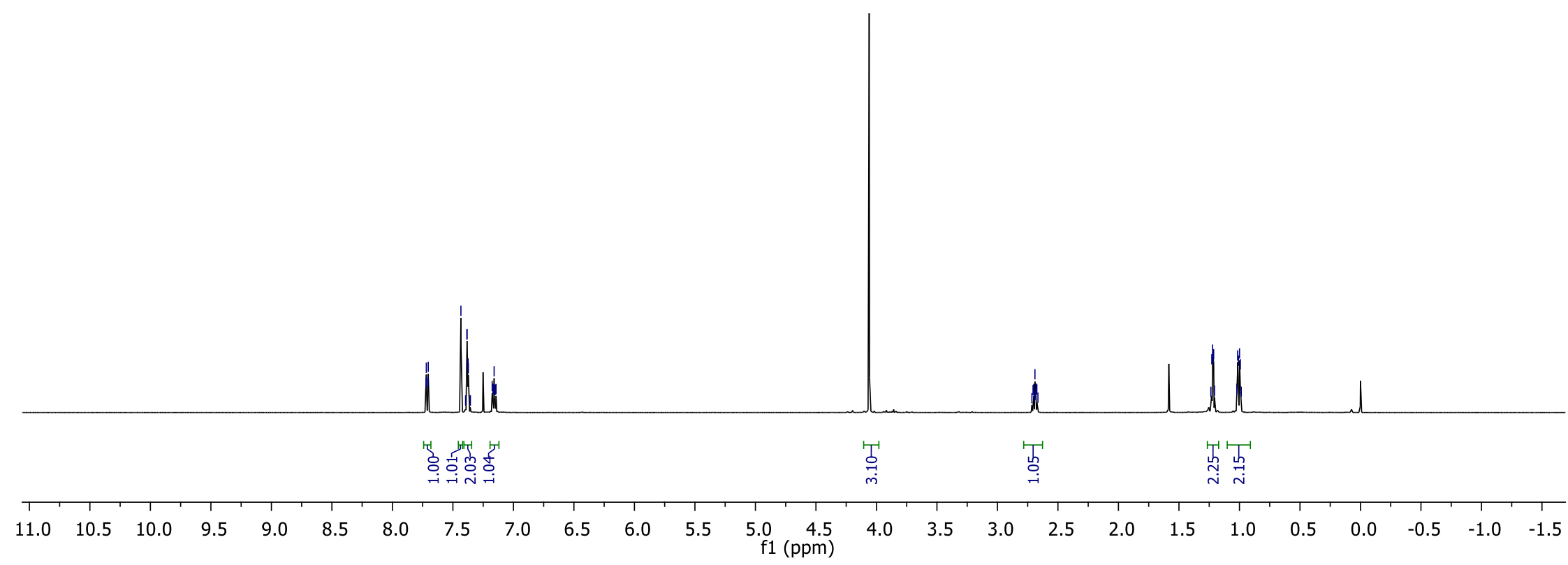



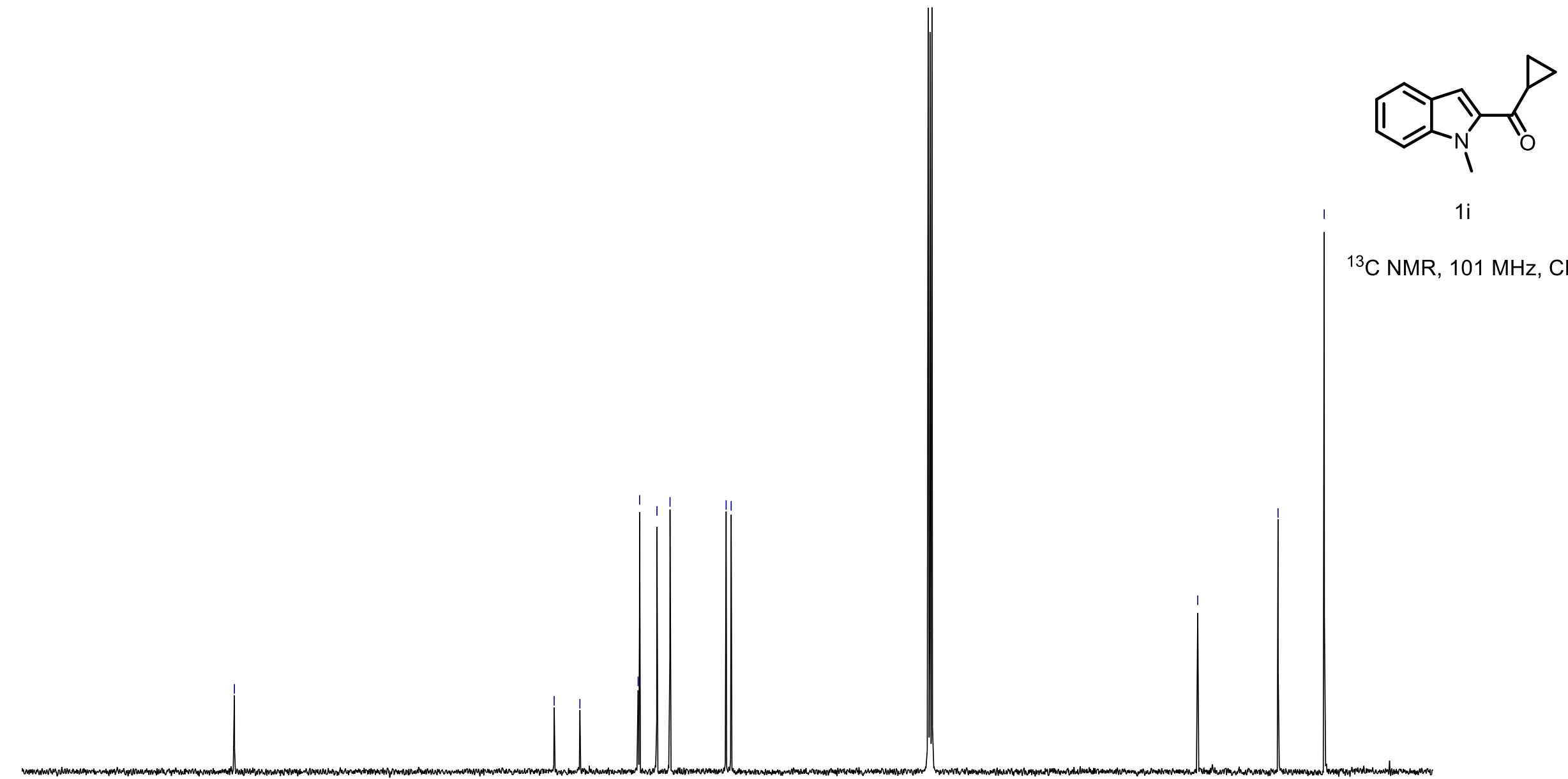

$1 \mathrm{i}$

${ }^{13} \mathrm{C}$ NMR, $101 \mathrm{MHz}, \mathrm{CDCl}_{3}$

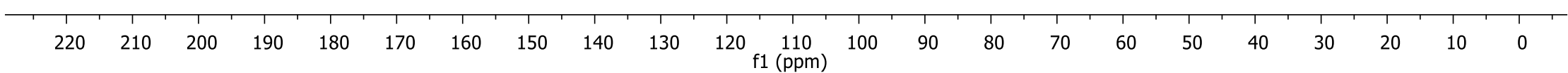




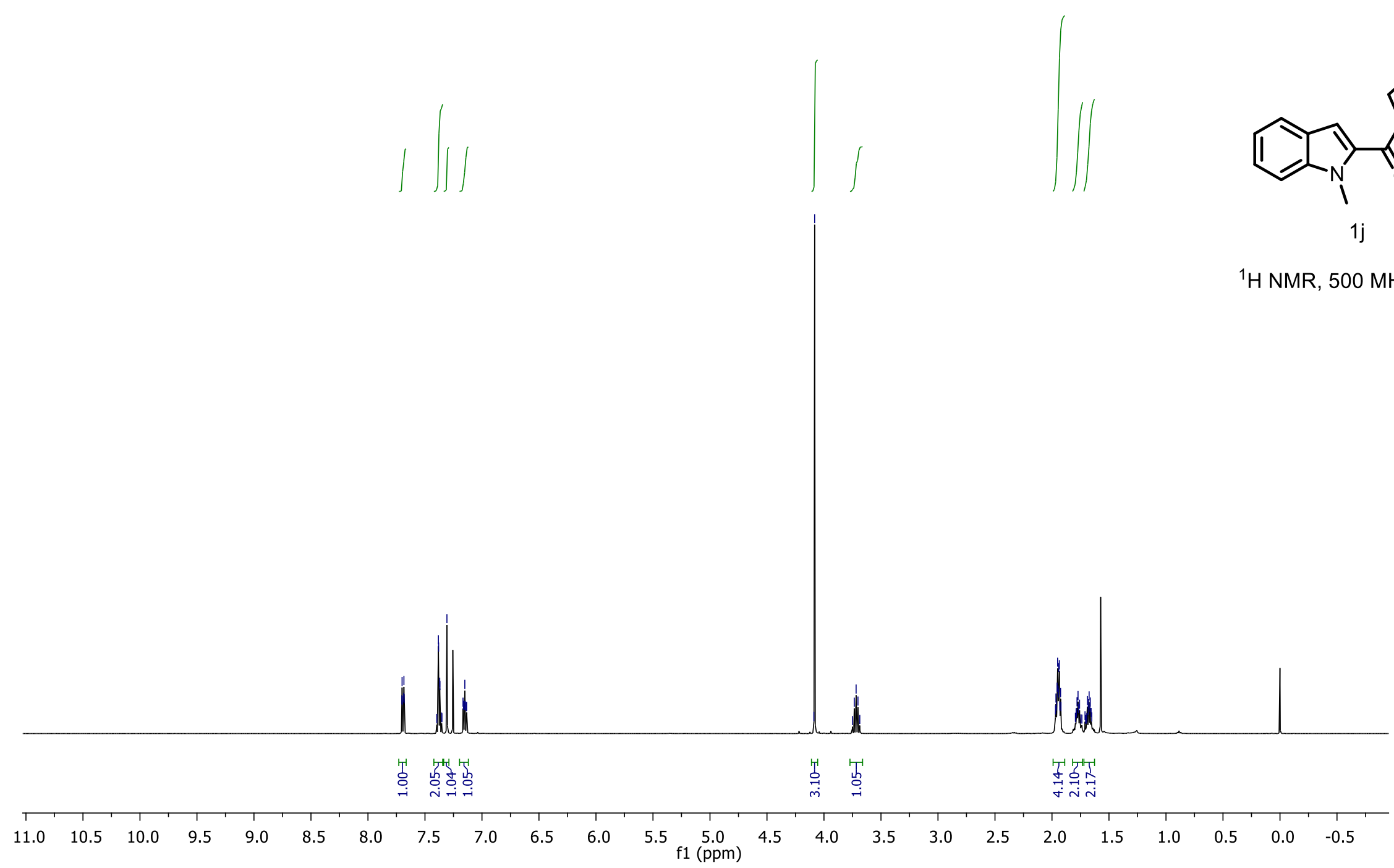




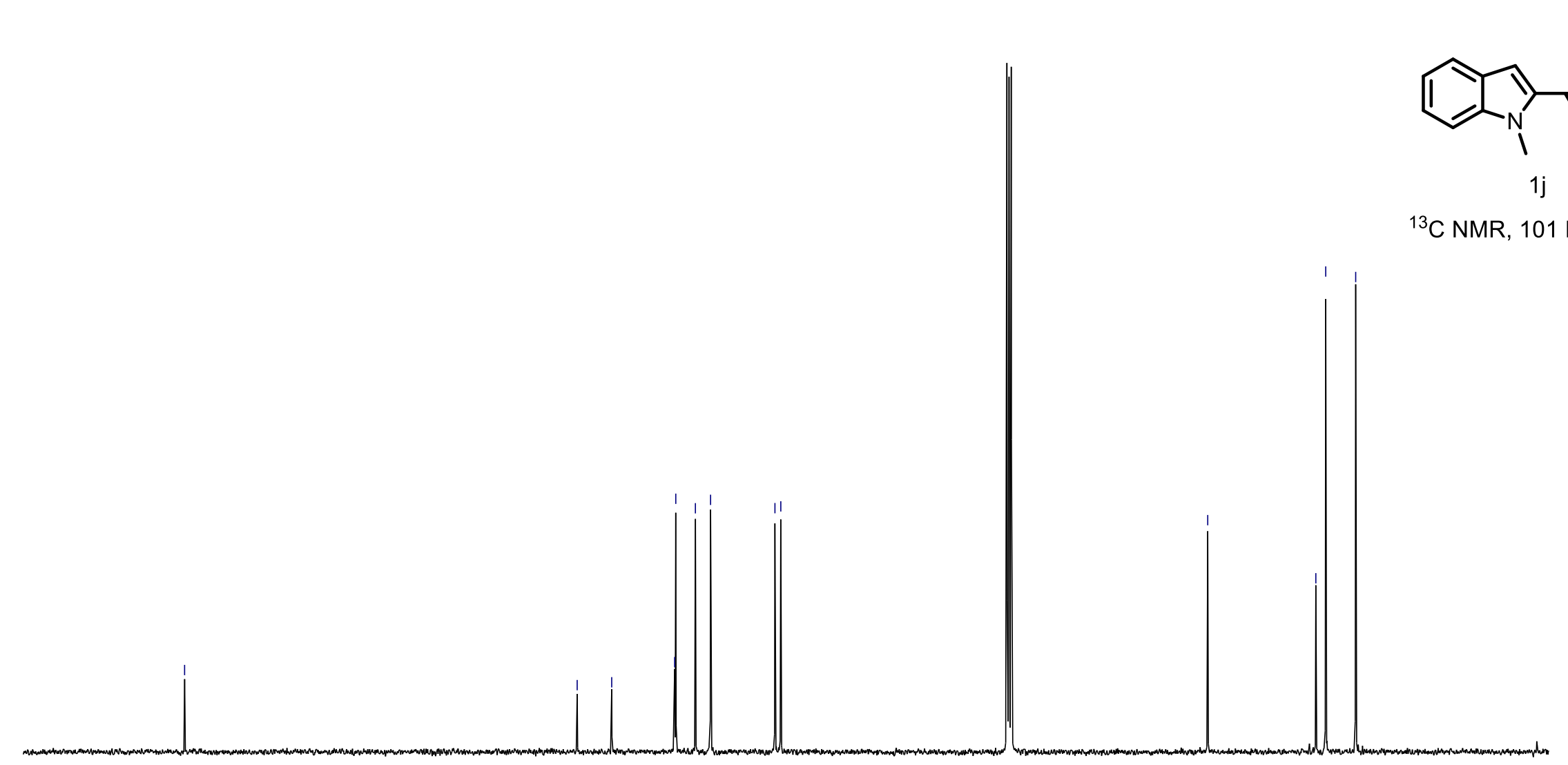

T⿱1 $220 \quad 210$ 


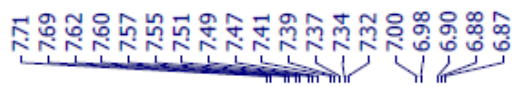

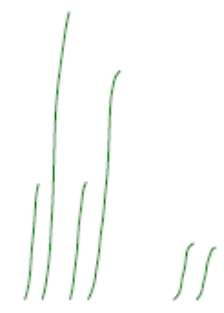

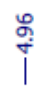

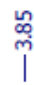

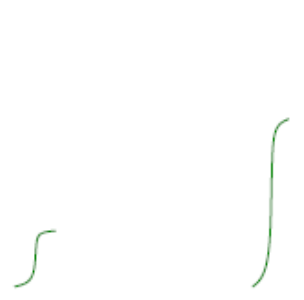

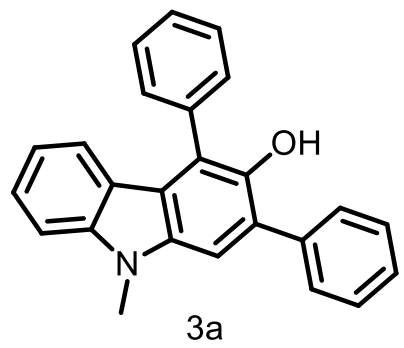

${ }^{1} \mathrm{H}$ NMR, $400 \mathrm{MHz}, \mathrm{CDCl}_{3}$

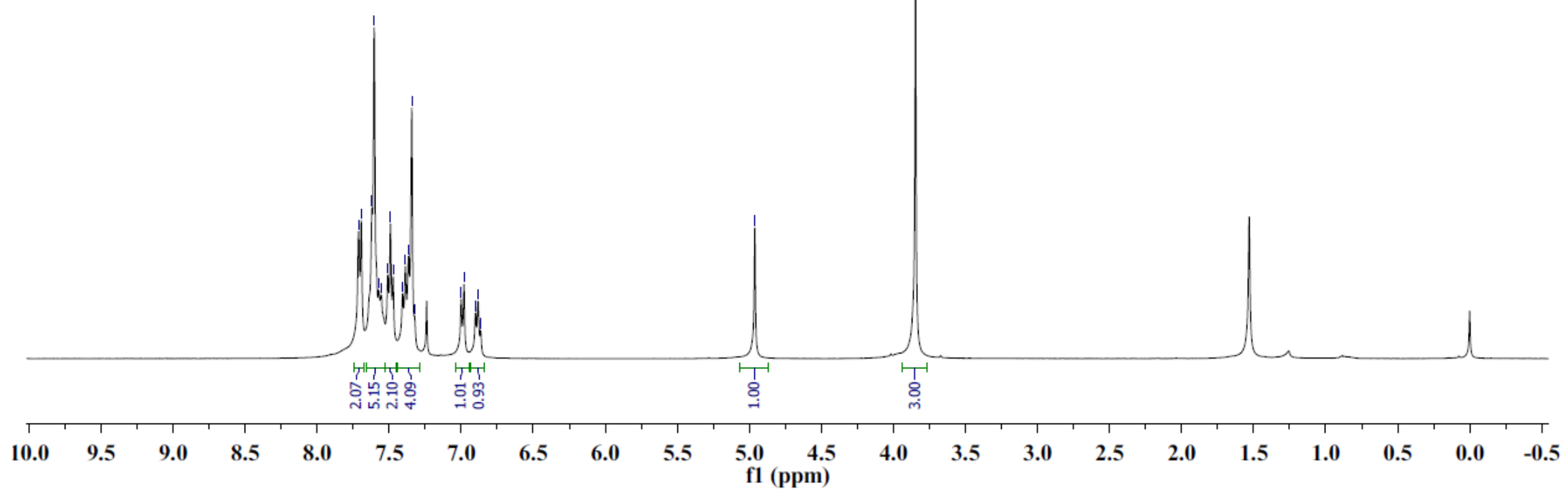

543 

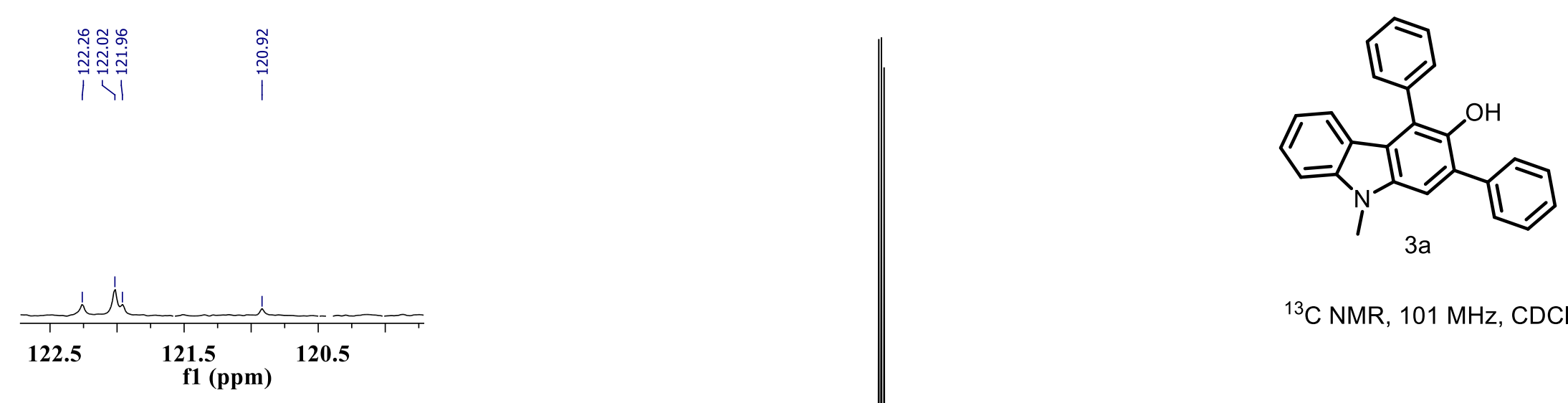

${ }^{13} \mathrm{C}$ NMR, $101 \mathrm{MHz}, \mathrm{CDCl}_{3}$

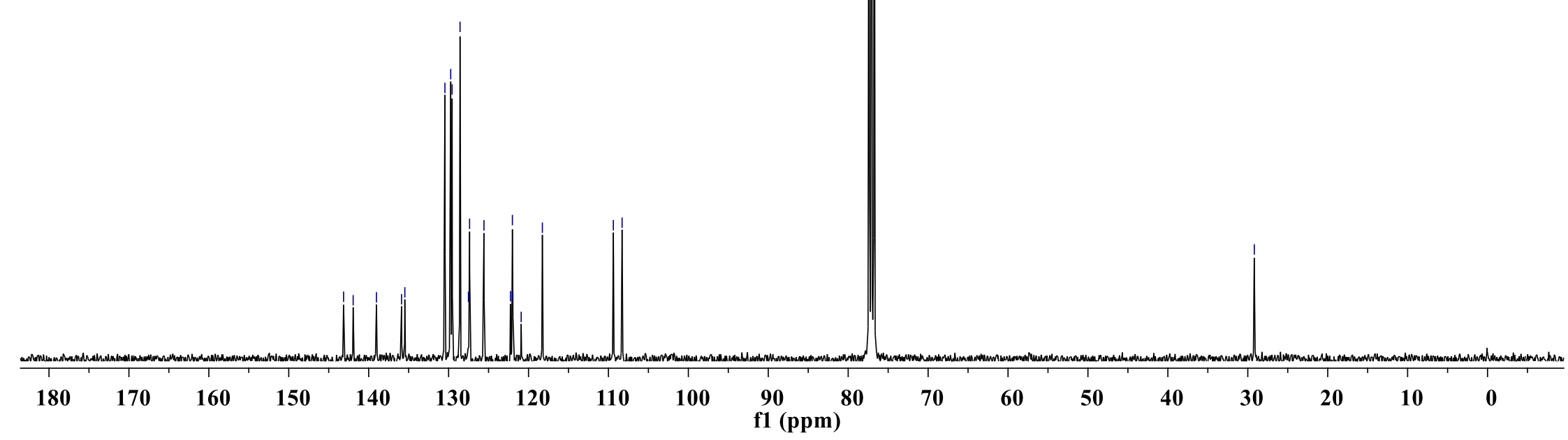




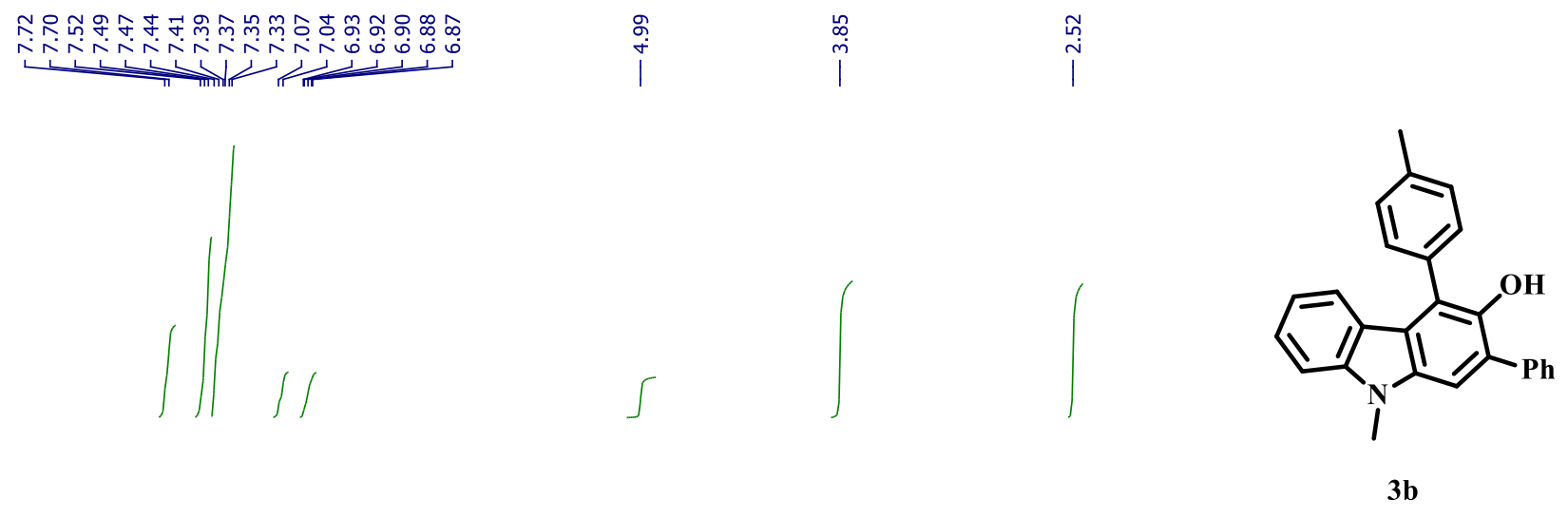

${ }^{1} \mathrm{H}$ NMR, $300 \mathrm{MHz}, \mathrm{CDCl}_{3}$

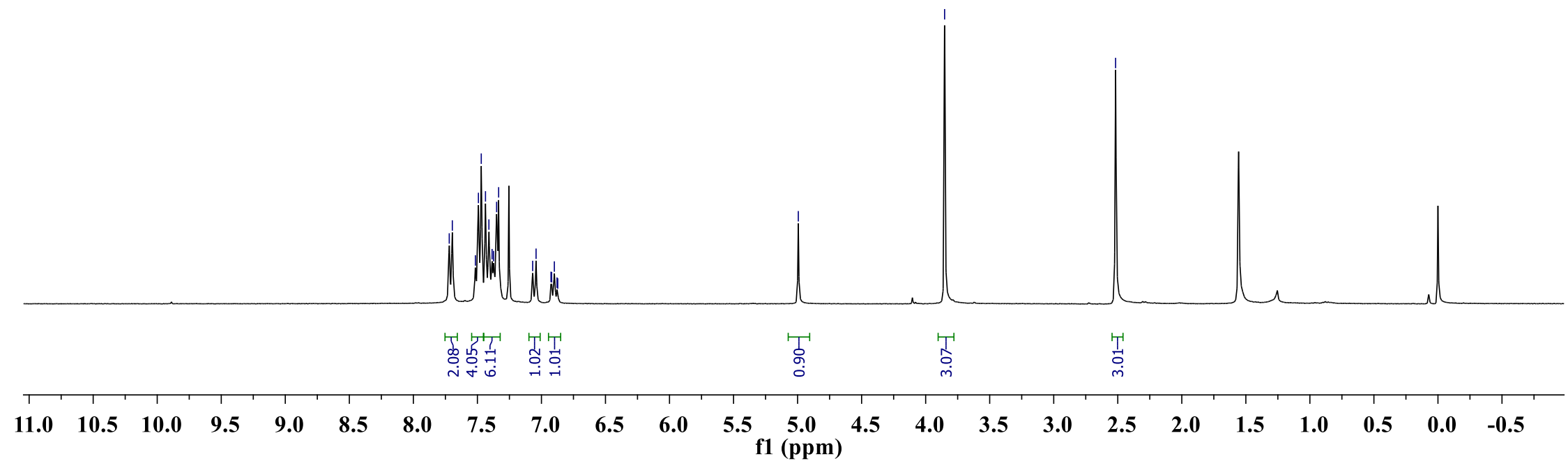




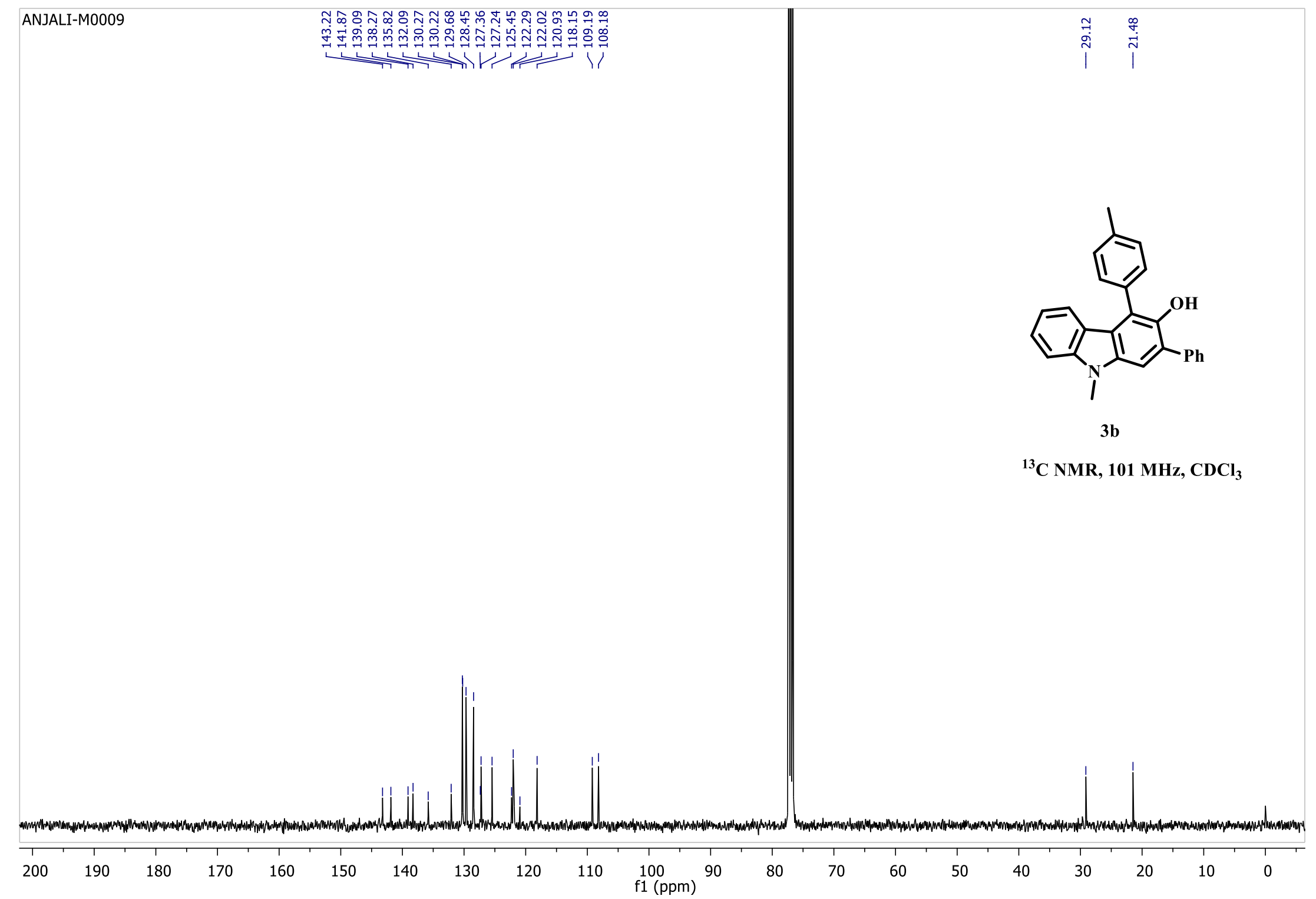



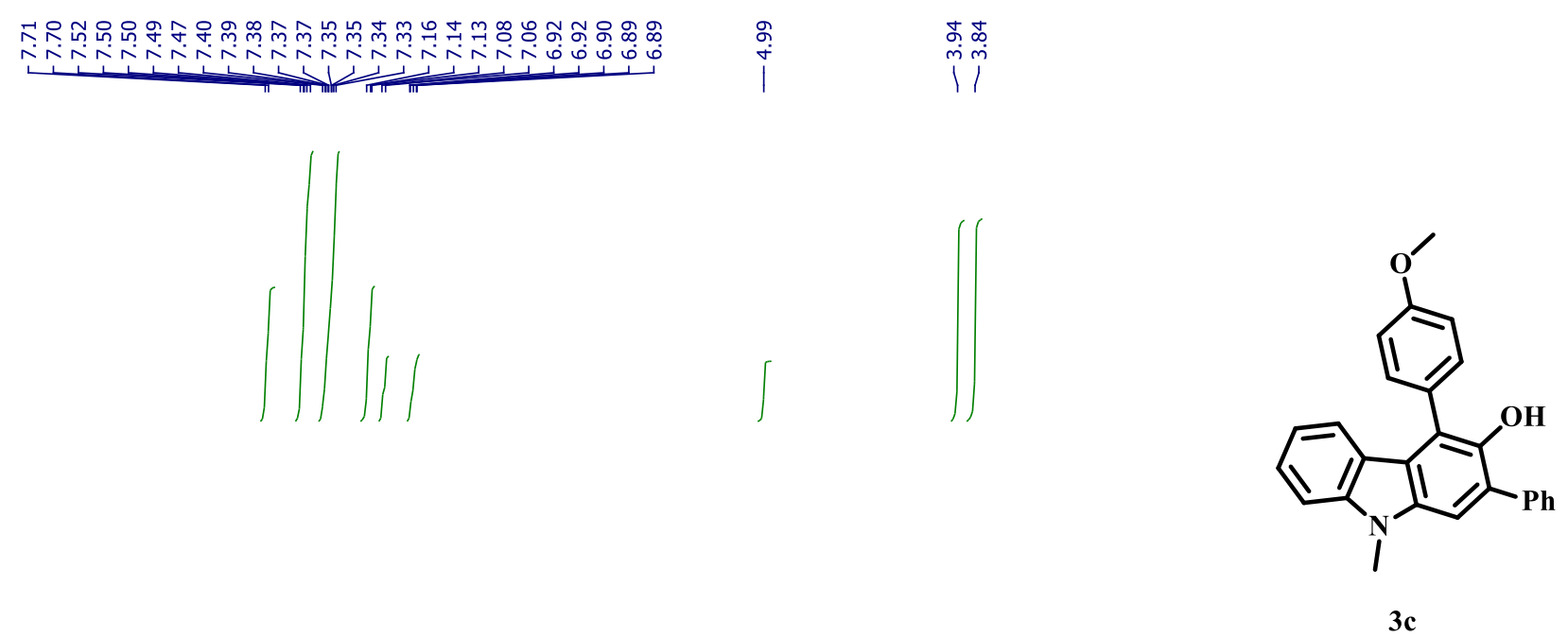

${ }^{1} \mathrm{H}_{\mathrm{NMR}}, 400 \mathrm{MHz}, \mathrm{CDCl}_{3}$

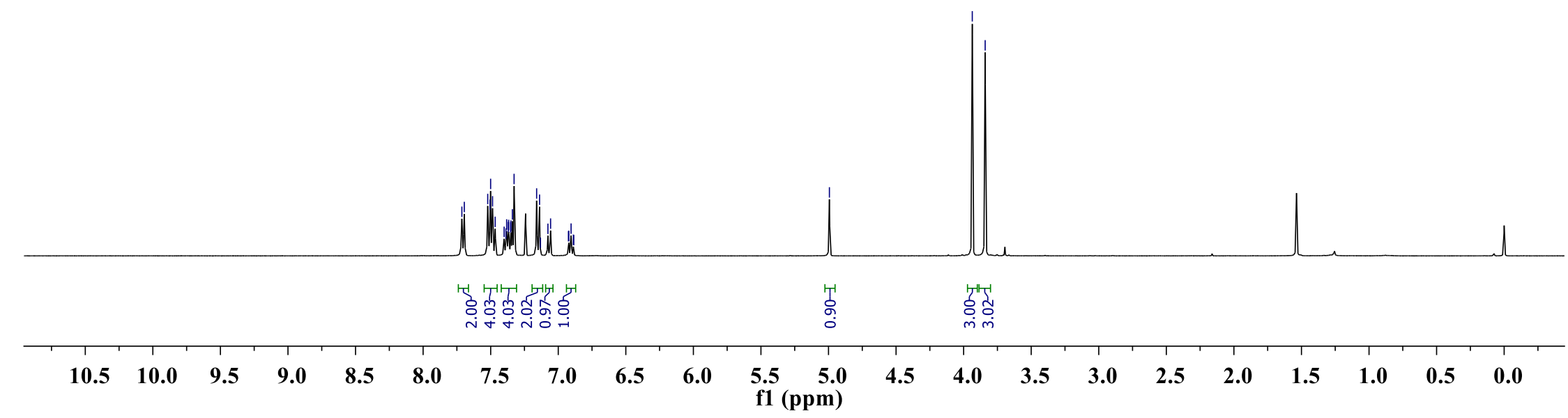




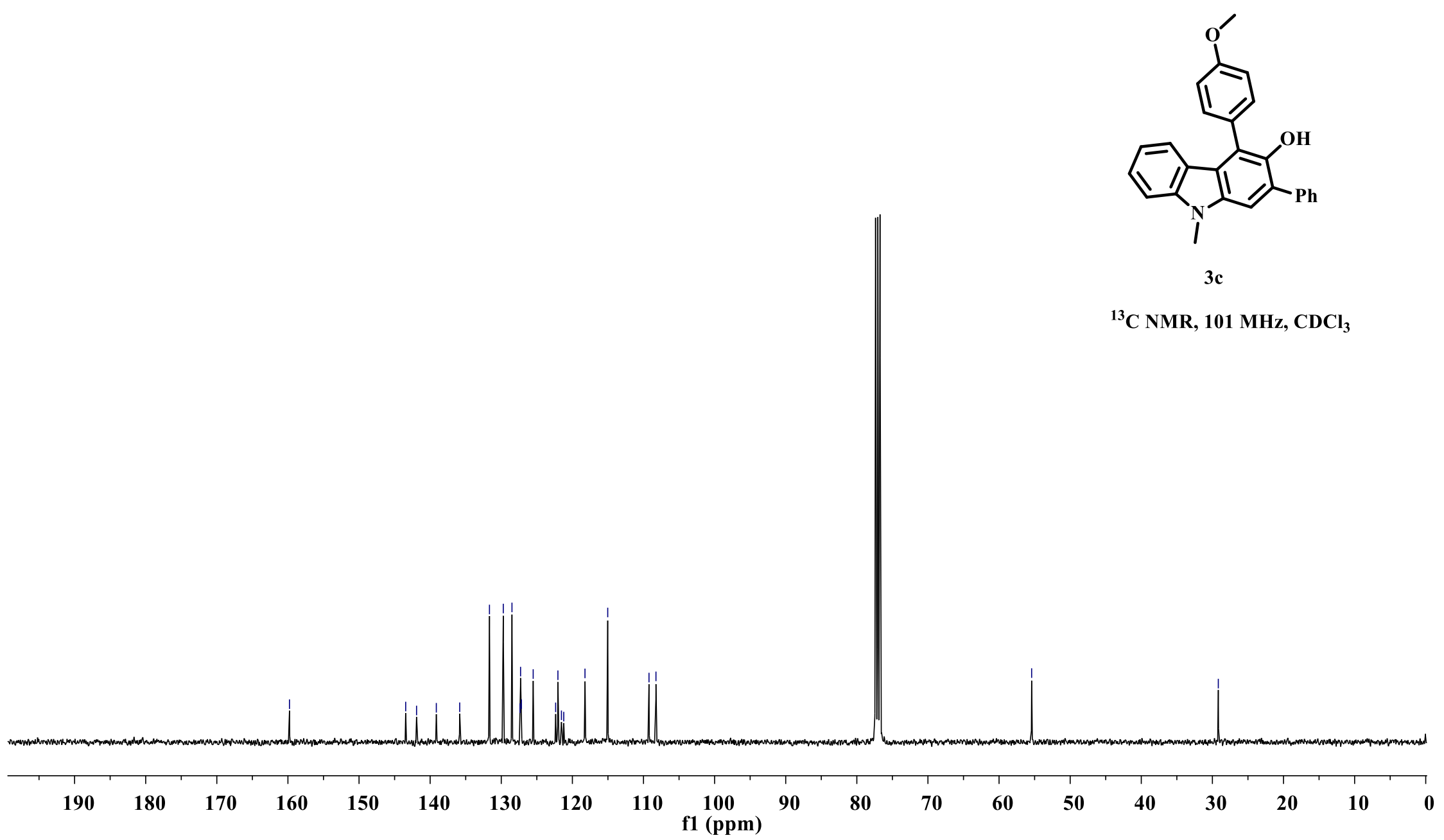


管

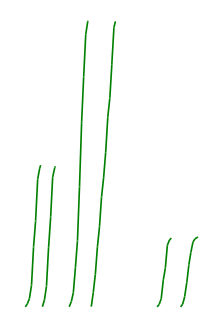

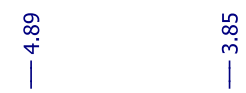

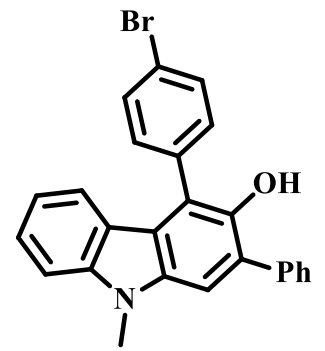

${ }^{1} \mathrm{H}_{\mathrm{NMR}}, 400 \mathrm{MHz}, \mathrm{CDCl}_{3}$

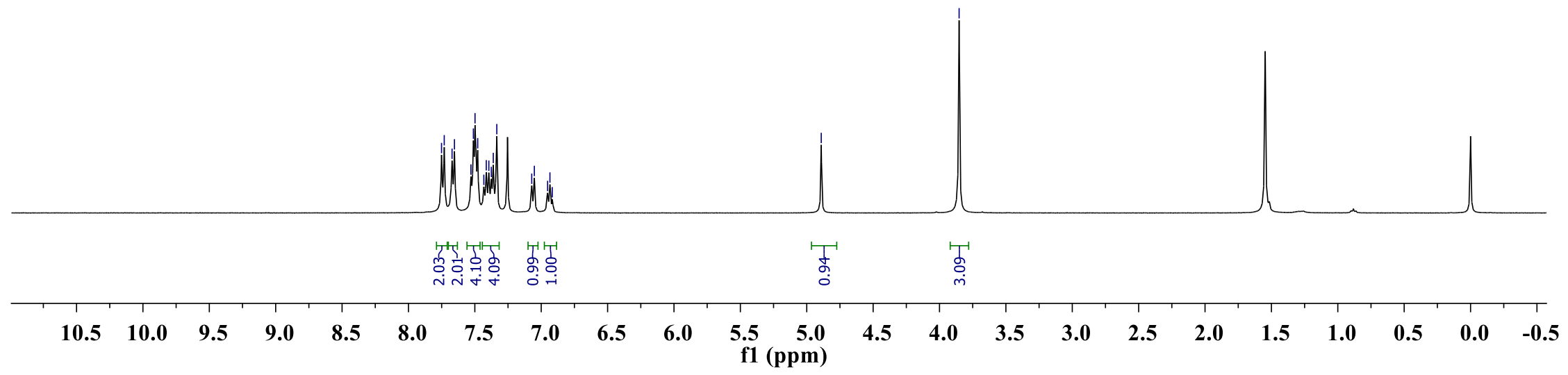




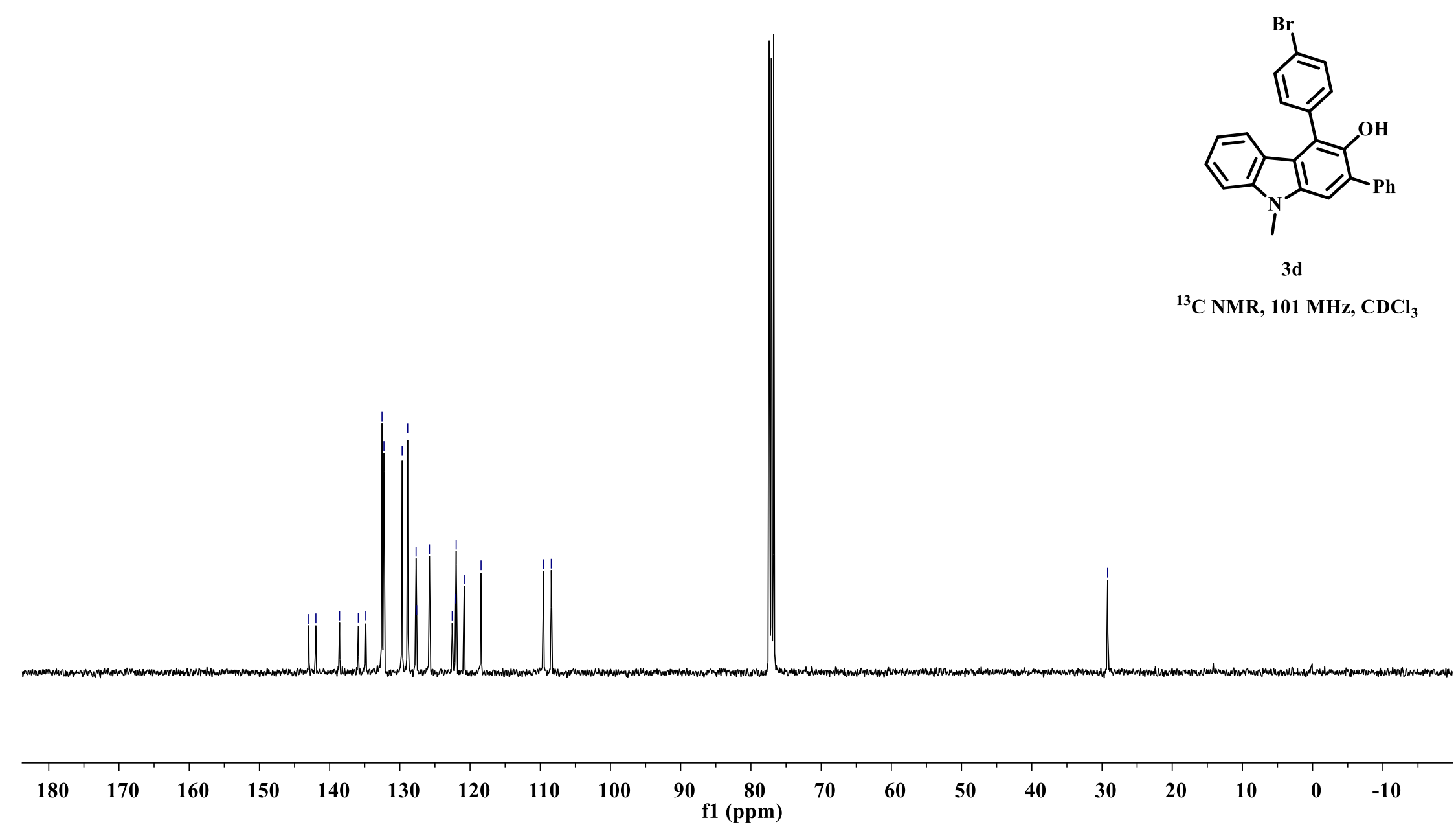




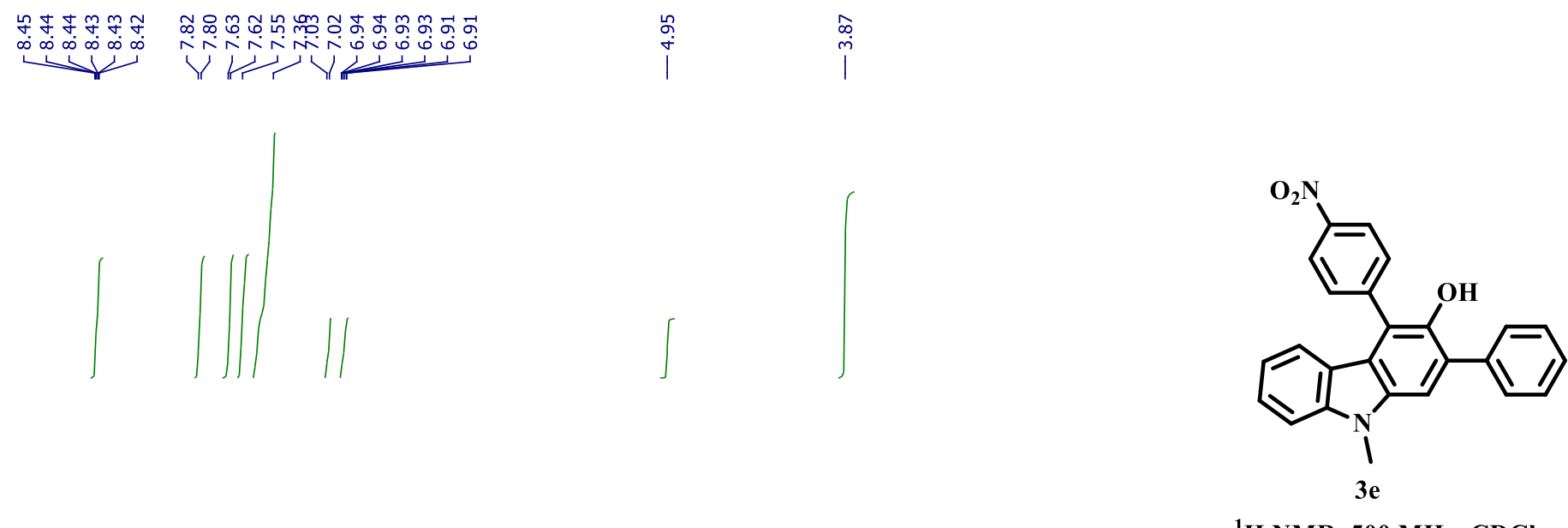

${ }^{1} \mathrm{H}$ NMR, $500 \mathrm{MHz}, \mathrm{CDCl}_{3}$

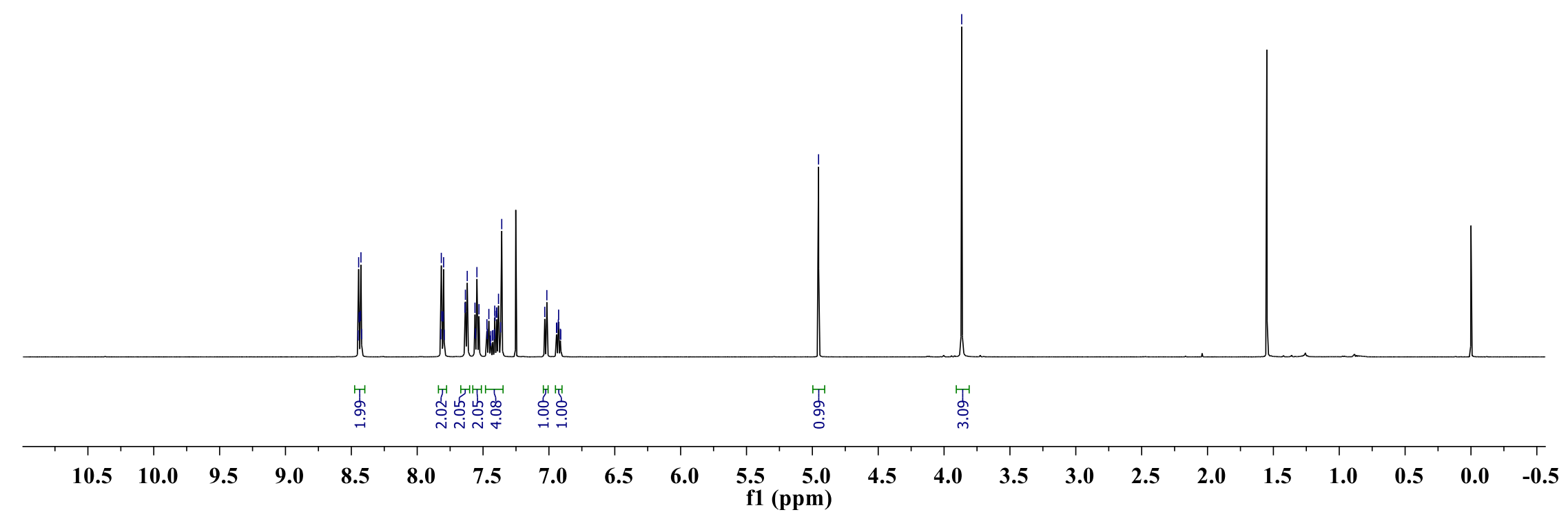




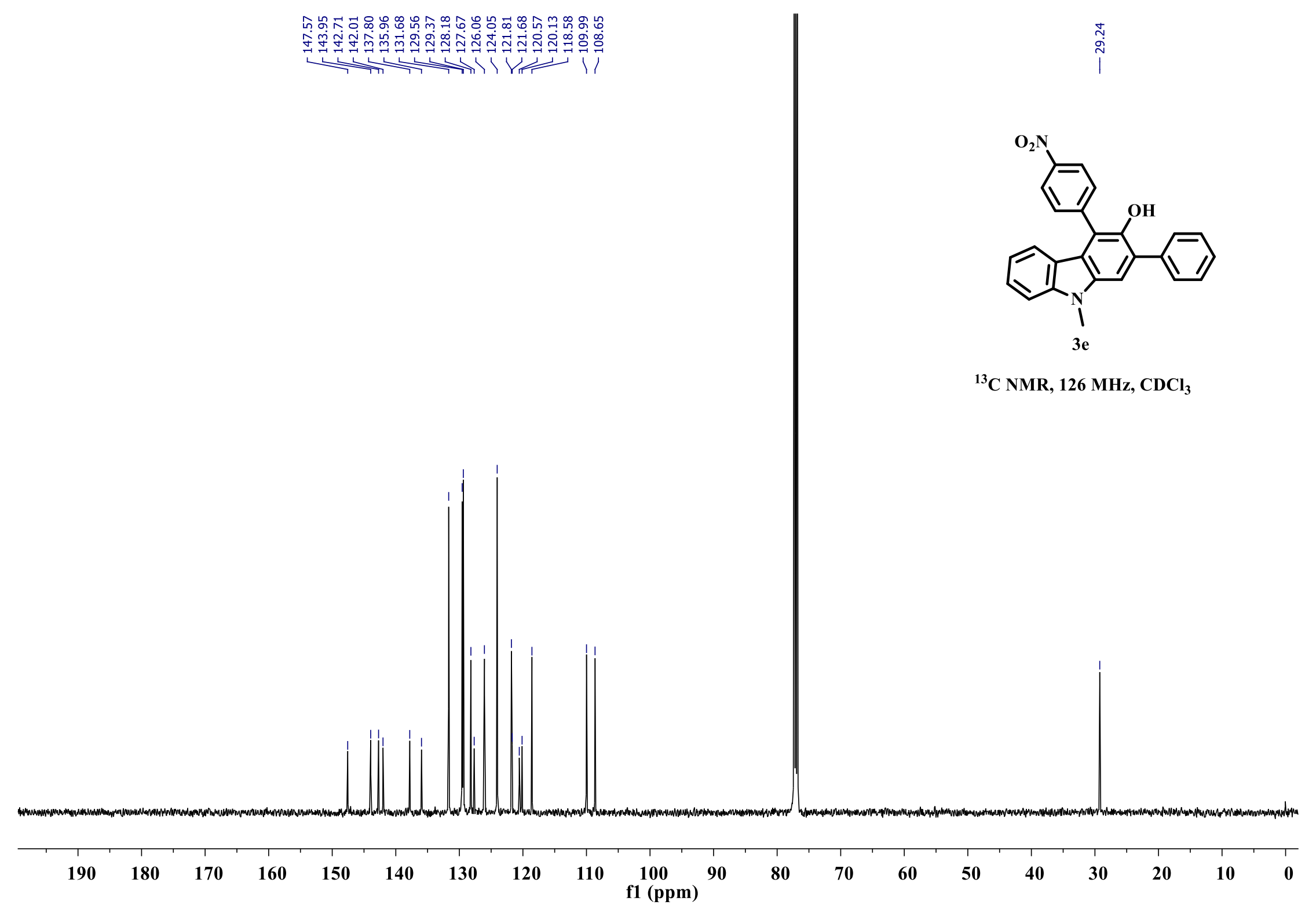




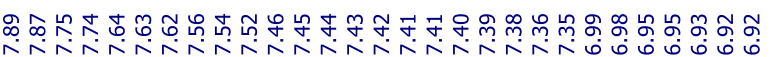
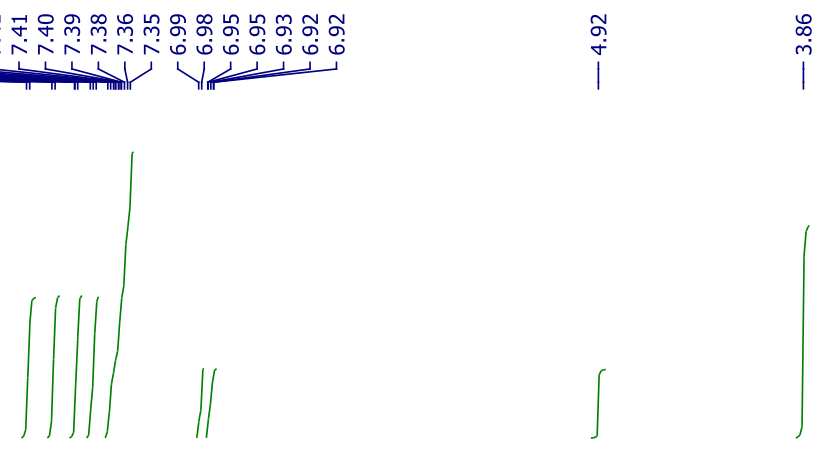

$\stackrel{\infty}{\infty}$

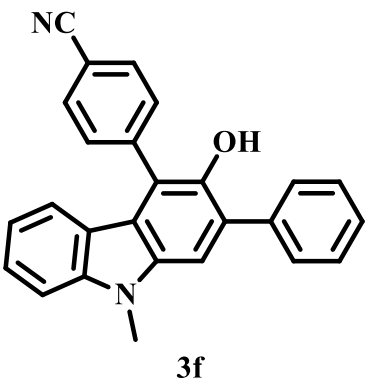

${ }^{1} \mathrm{H}$ NMR, $500 \mathrm{MHz}, \mathrm{CDCl}_{3}$

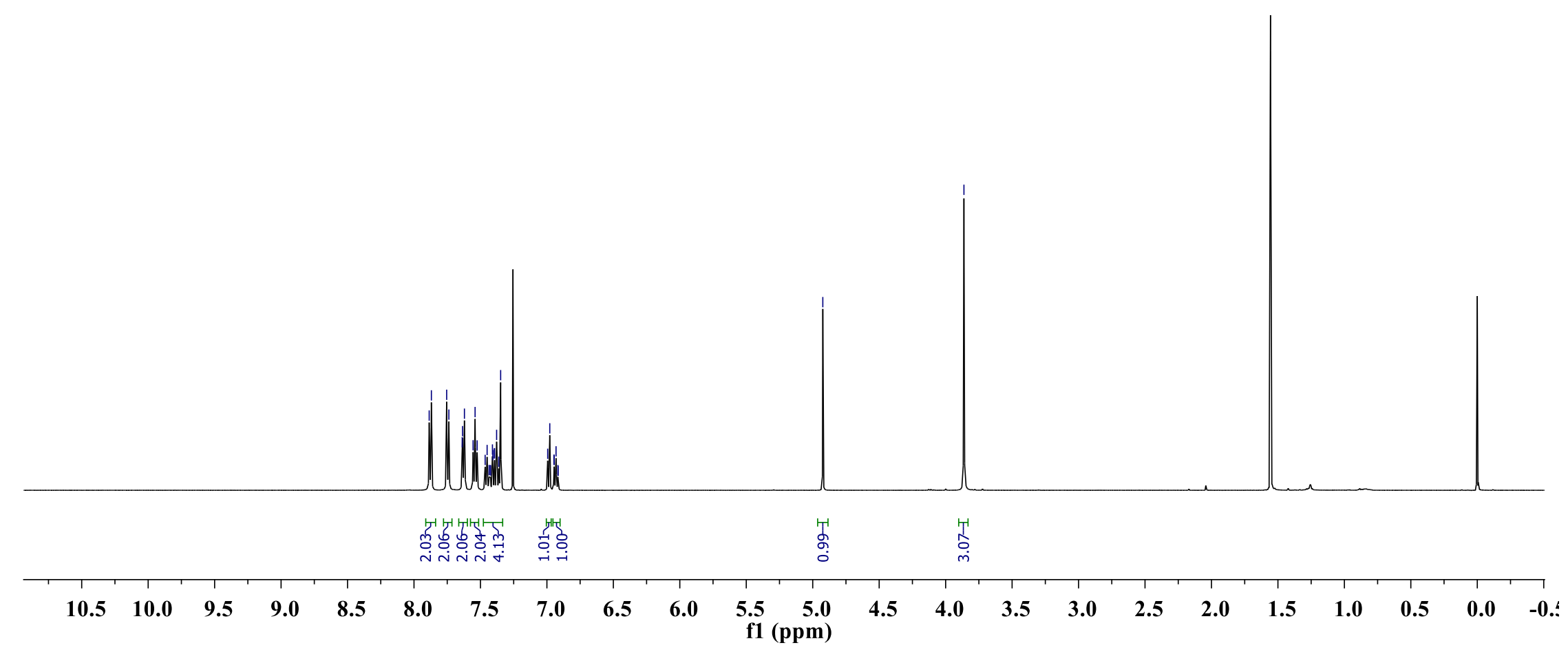




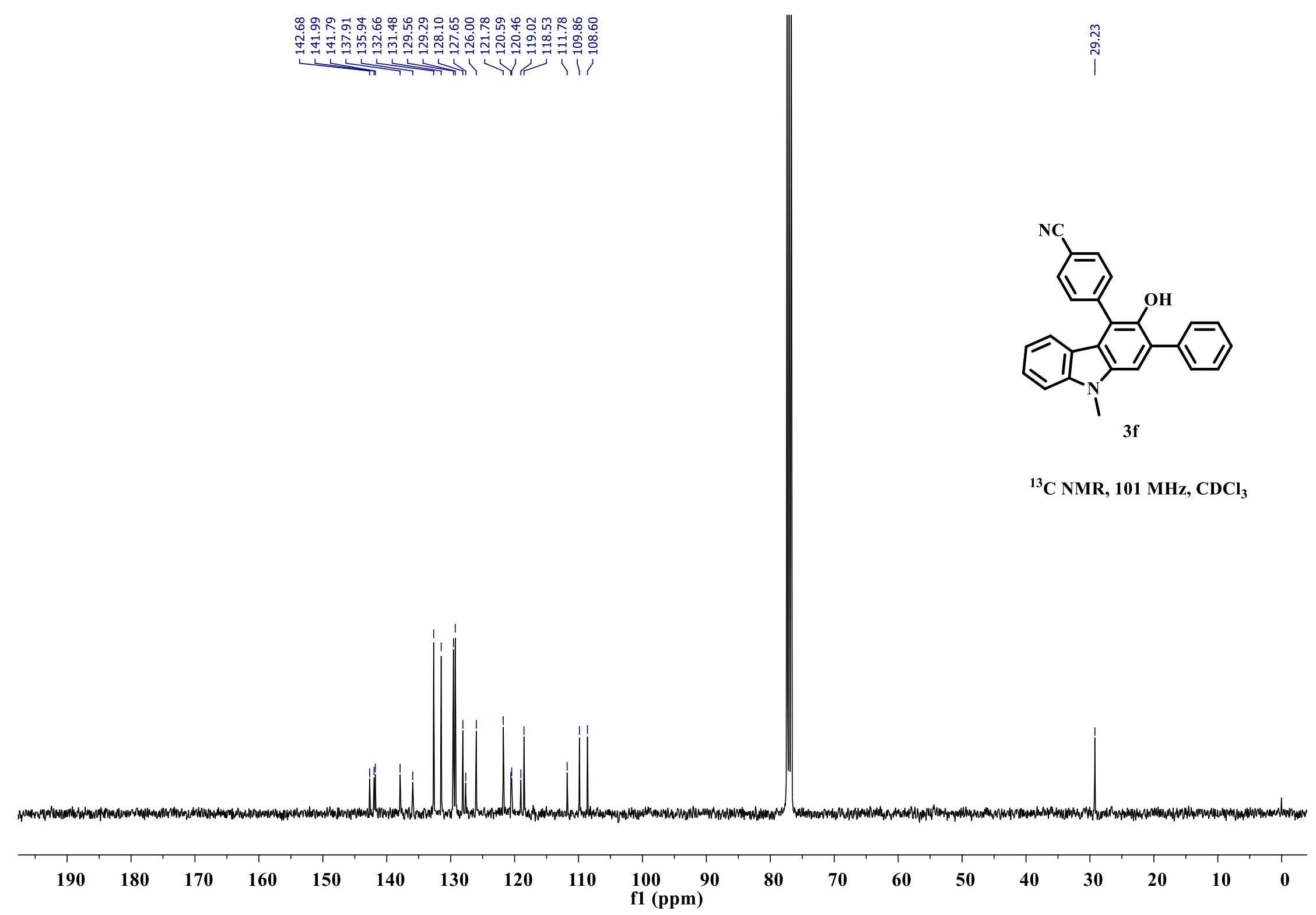




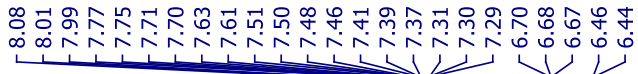

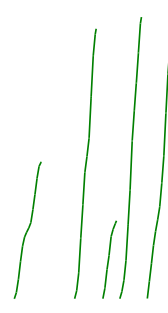

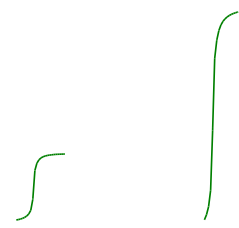

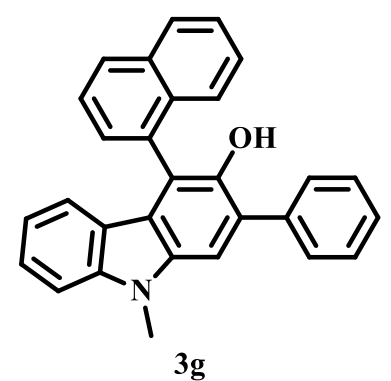

${ }^{1} \mathrm{H}$ NMR, $400 \mathrm{MHz}, \mathrm{CDCl}_{3}$

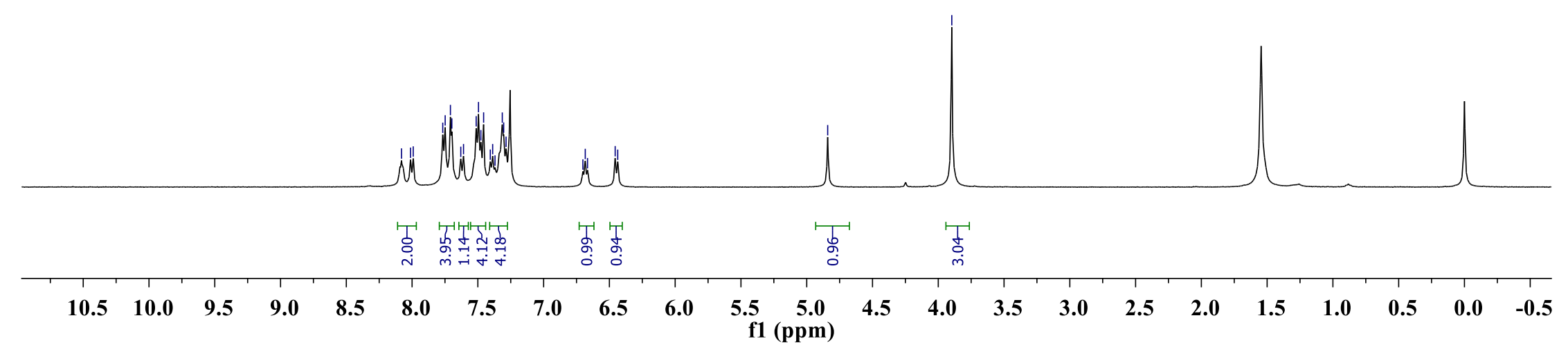




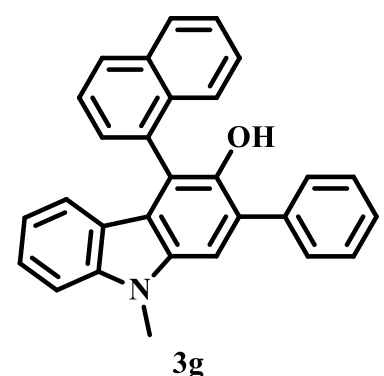

${ }^{13} \mathrm{C}$ NMR, $75 \mathrm{MHz} \mathrm{CDCl}_{3}$

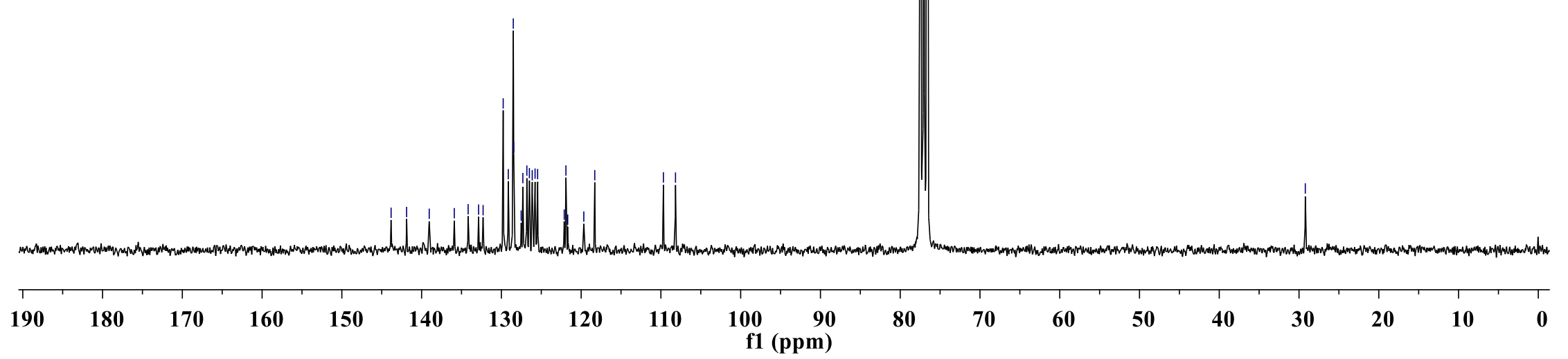



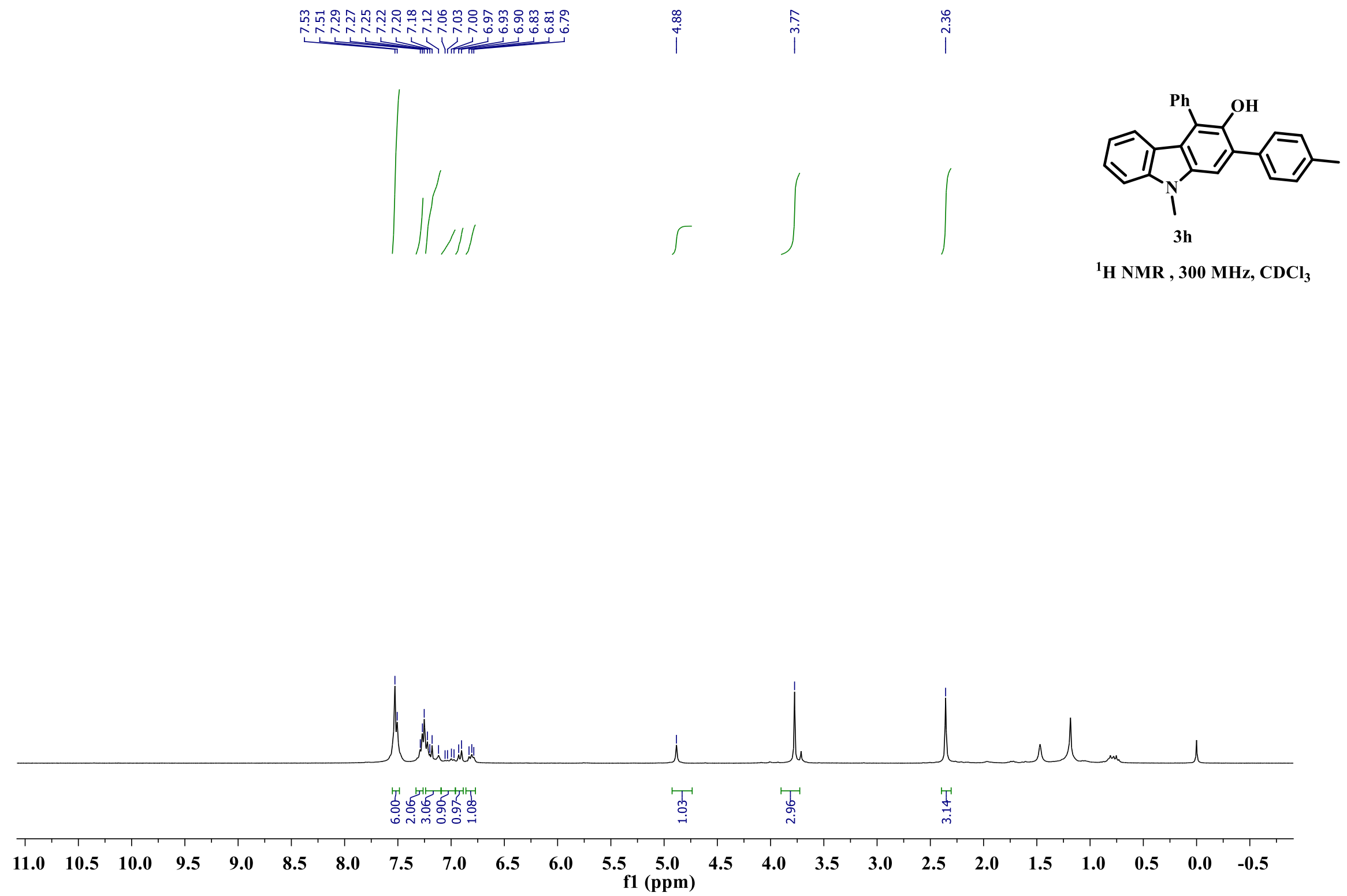


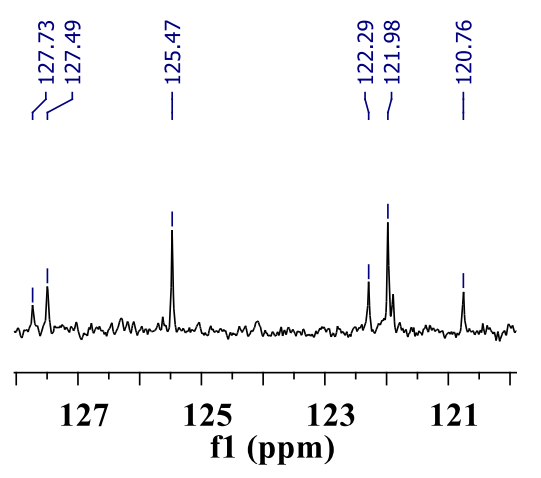

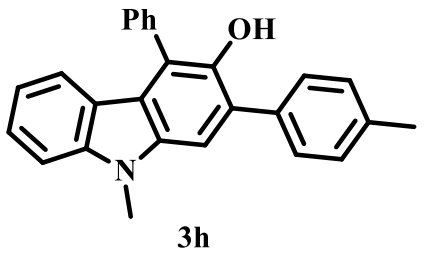

${ }^{13} \mathrm{C}$ NMR, $126 \mathrm{MHz}, \mathrm{CDCl}_{3}$

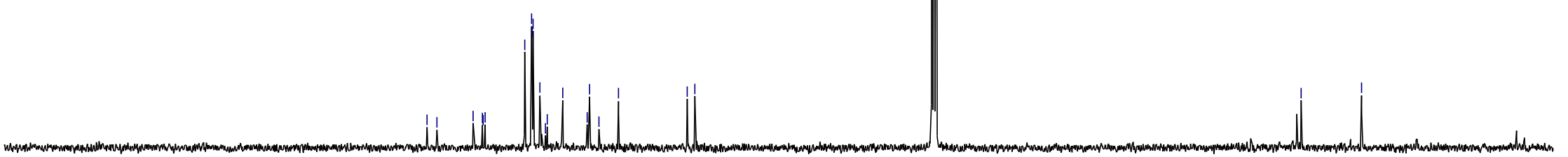

$\begin{array}{lllll}190 & 180 & 170 & 160 & 150\end{array}$

$140 \quad 130$ 


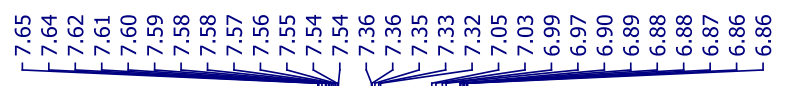

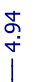

$\underbrace{\substack{\infty \\ j}}_{\substack{\infty \\ i \infty}}$
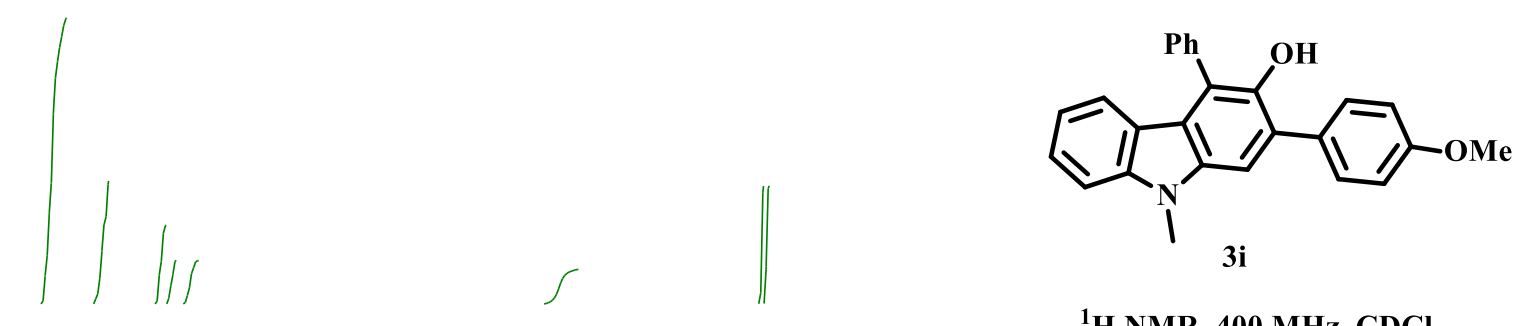

${ }^{1} \mathrm{H}$ NMR, $400 \mathrm{MHz}, \mathrm{CDCl}_{3}$

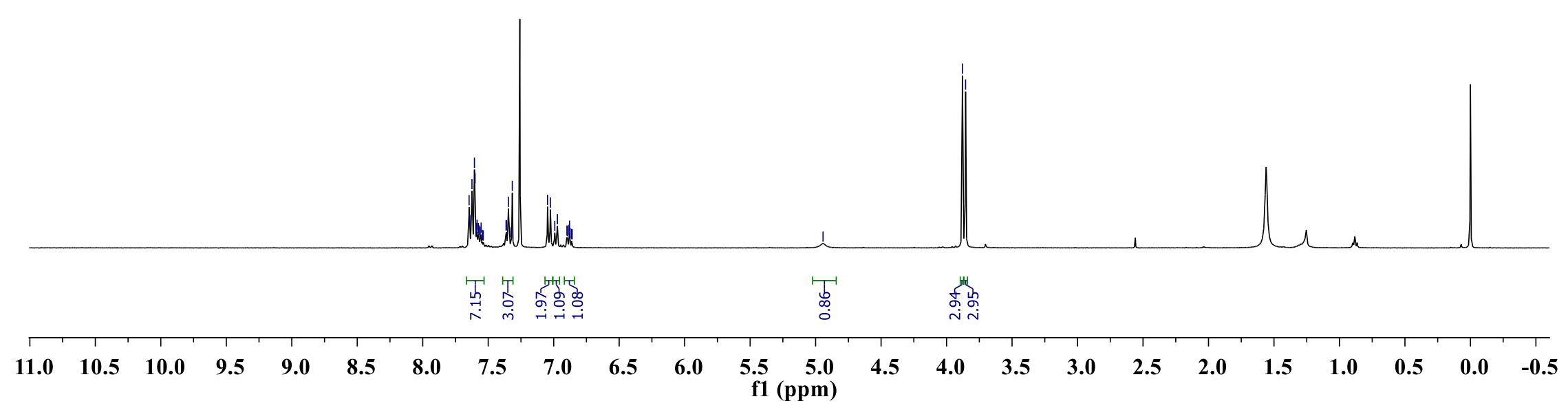



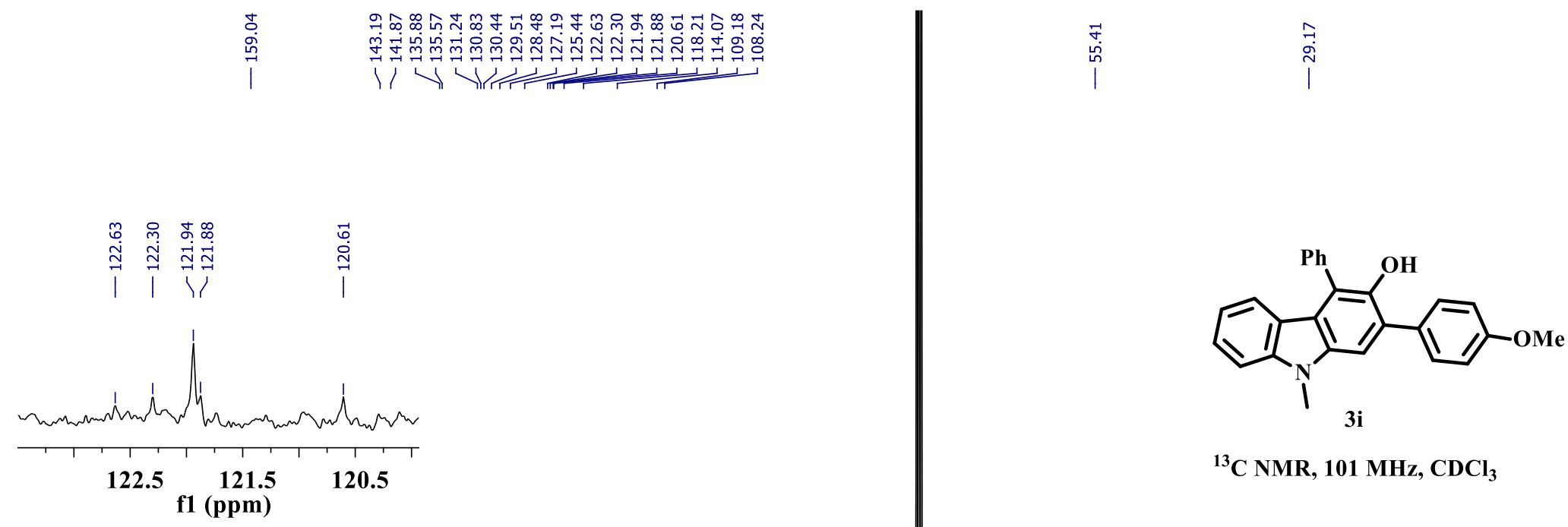

${ }^{13} \mathrm{C}$ NMR, $101 \mathrm{MHz}, \mathrm{CDCl}_{3}$

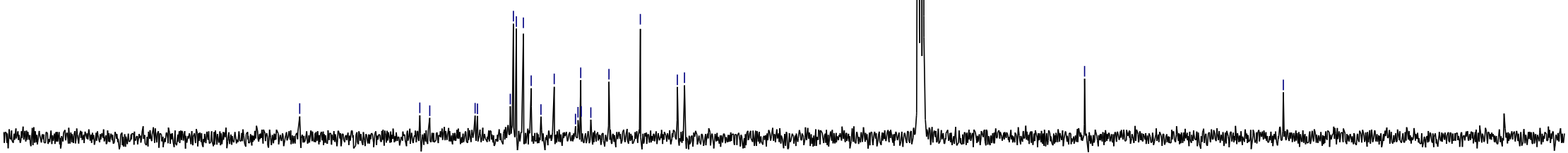

$\begin{array}{llllllllllllllllllll}190 & 180 & 170 & 160 & 150 & 140 & 130 & 120 & 110 & \begin{array}{r}100 \\ \text { f1 }(\mathrm{ppm})\end{array} & 80 & 70 & 60 & 50 & 40 & 30 & 20 & 10 & 0\end{array}$



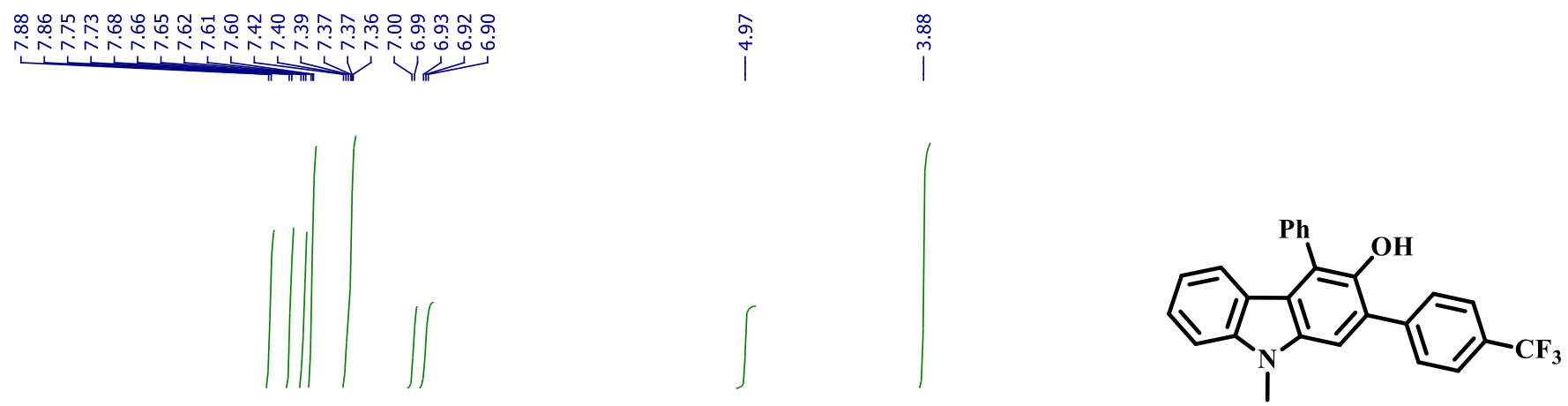

${ }^{1} \mathrm{H}$ NMR, $500 \mathrm{MHz}, \mathrm{CDCl}_{3}$

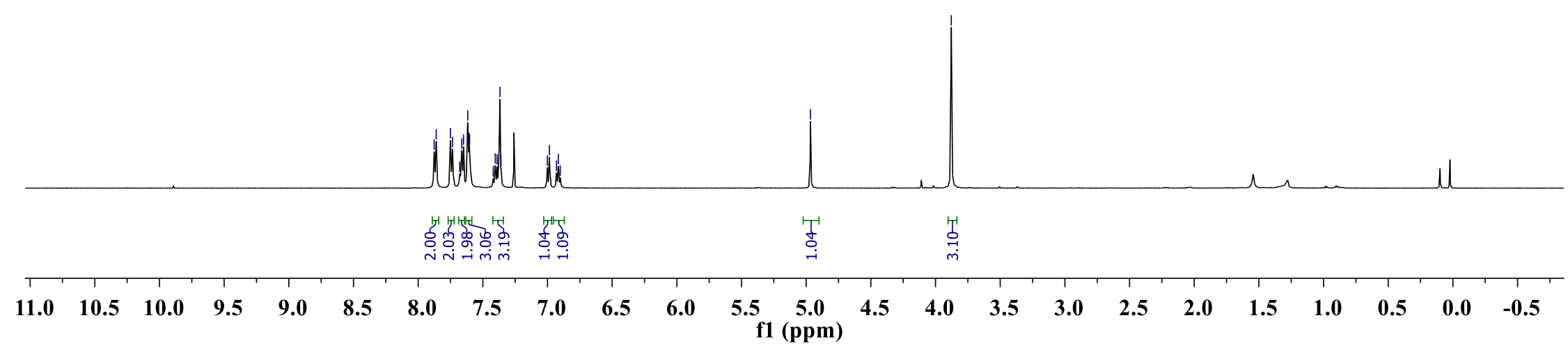




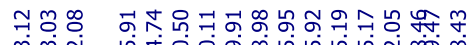

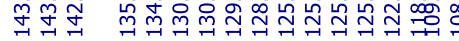 \\ i) I)}

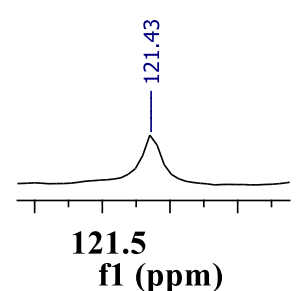

f1 (ppm)

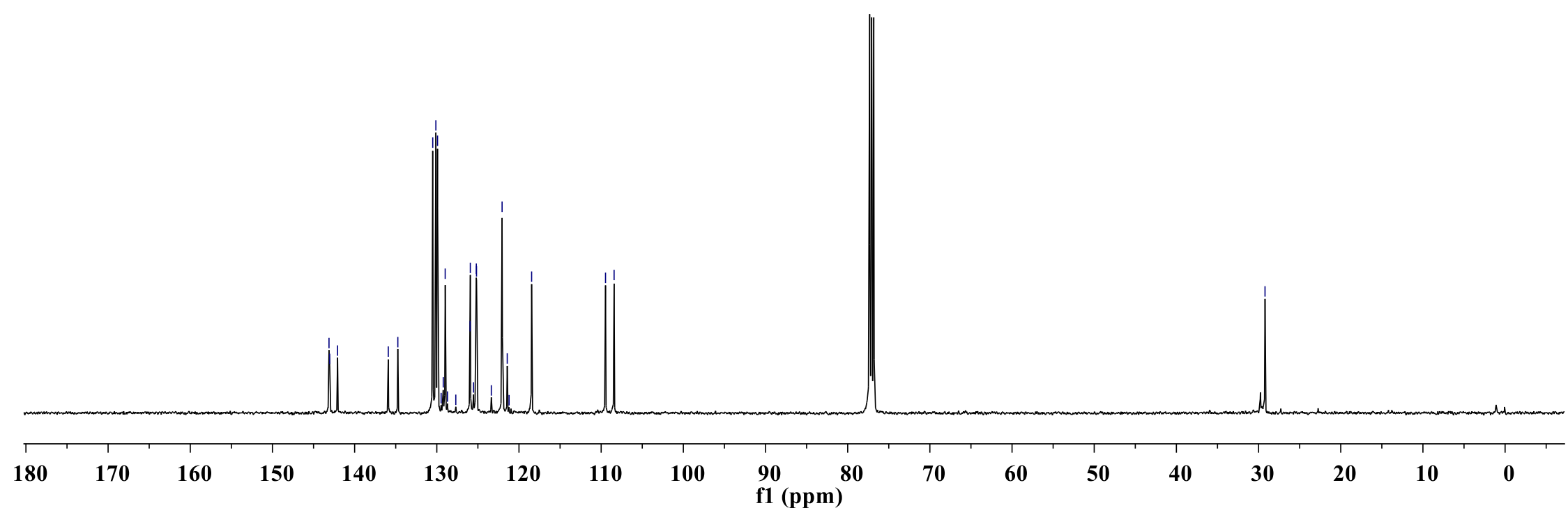

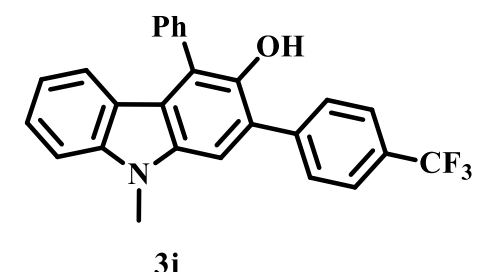

${ }^{13} \mathrm{C}$ NMR, $126 \mathrm{MHz}, \mathrm{CDCl}_{3}$ 


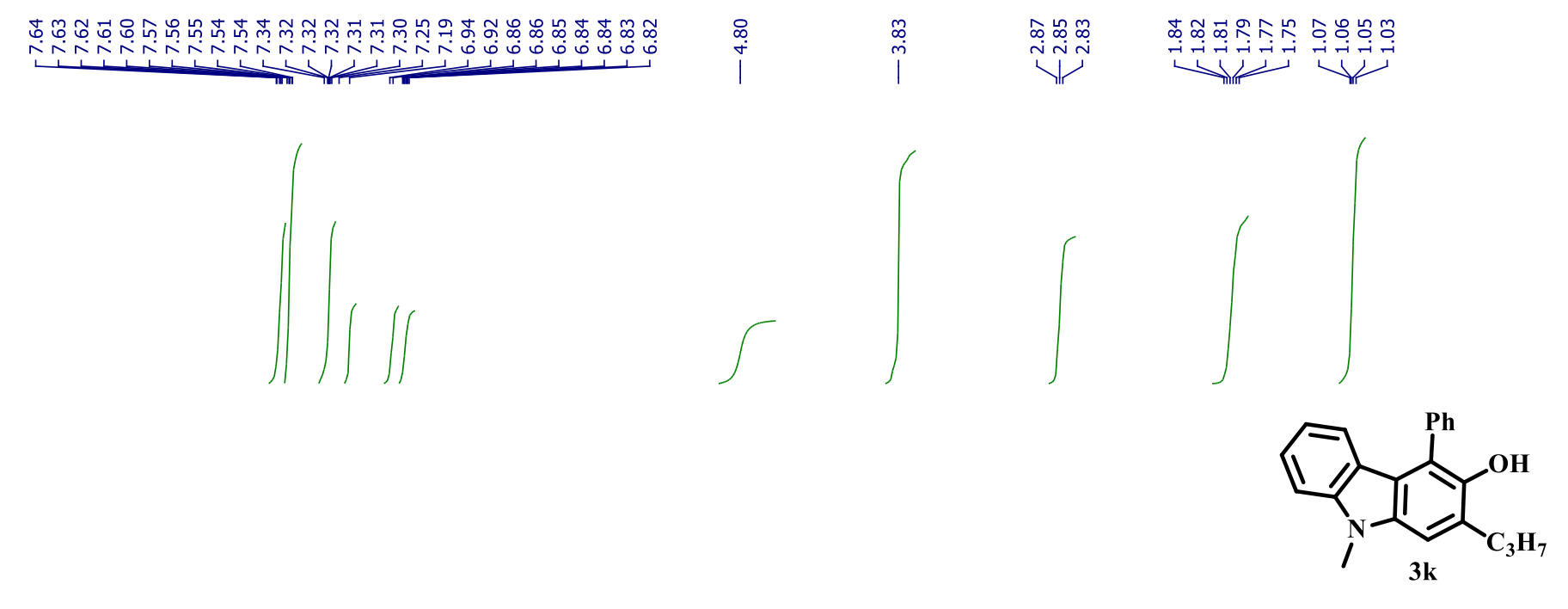

${ }^{1} \mathrm{H}$ NMR, $400 \mathrm{MHz}, \mathrm{CDCl}_{3}$

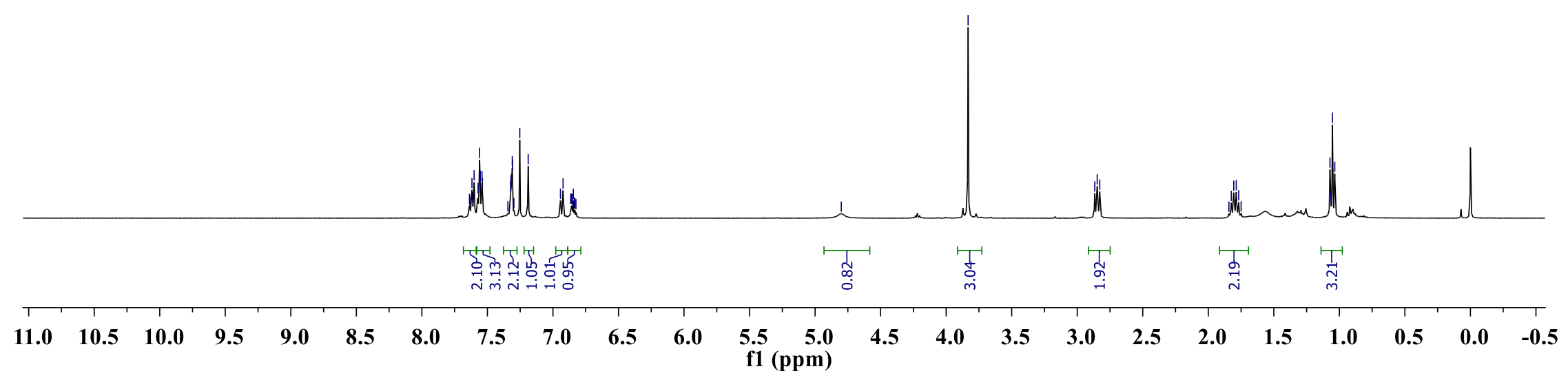




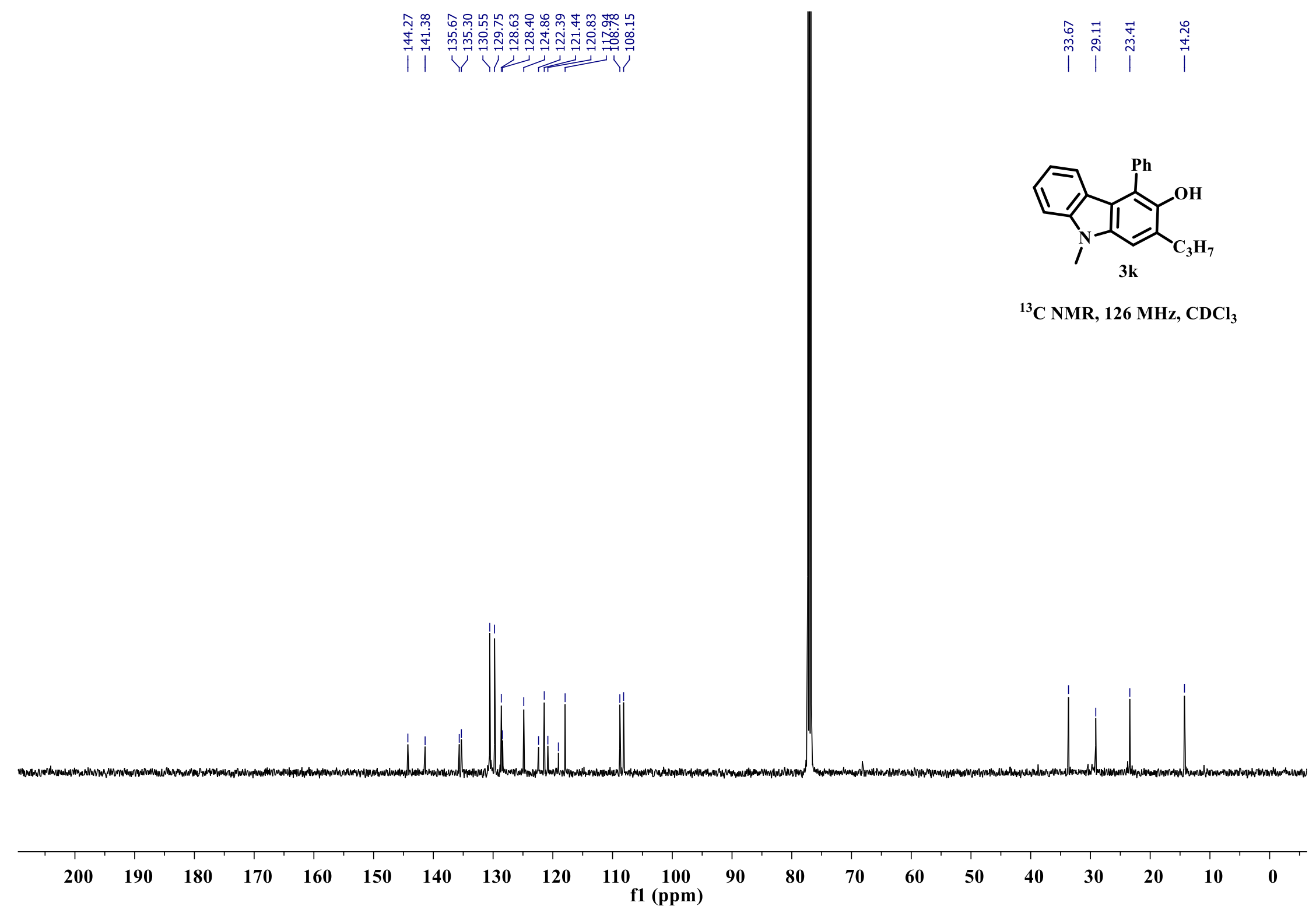




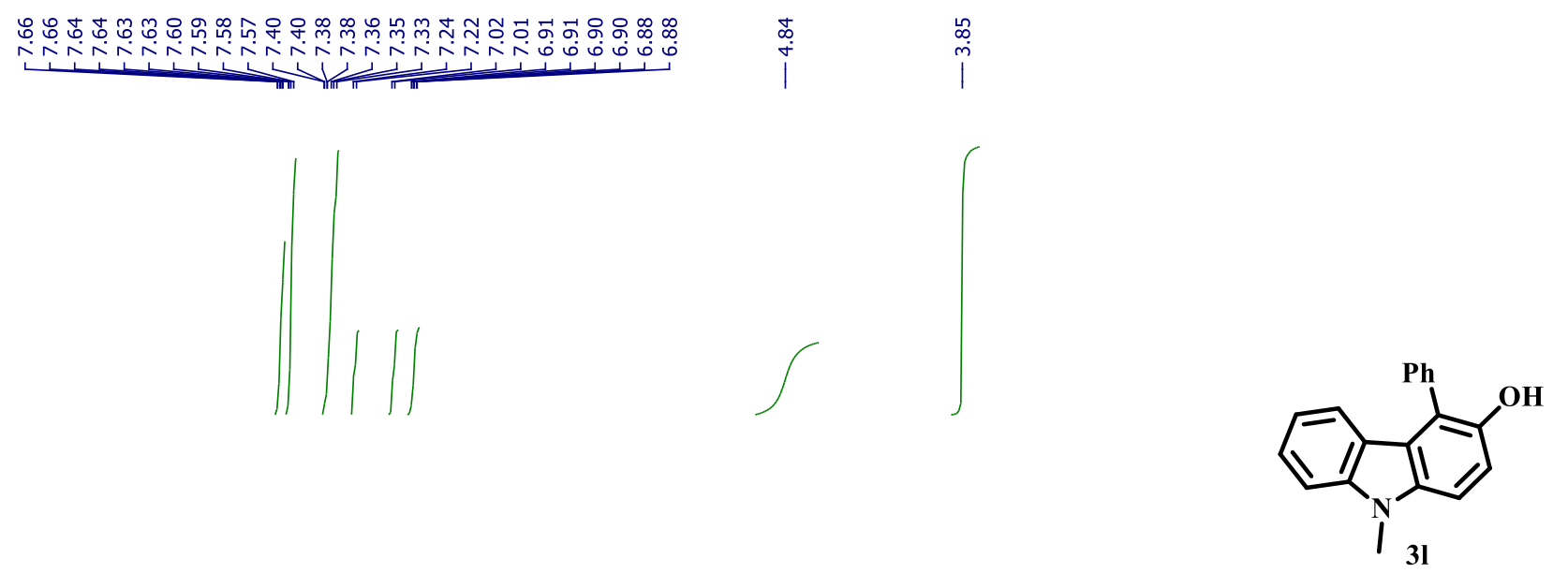

${ }^{1} \mathrm{H}$ NMR, $500 \mathrm{MHz} \mathrm{CDCl}_{3}$

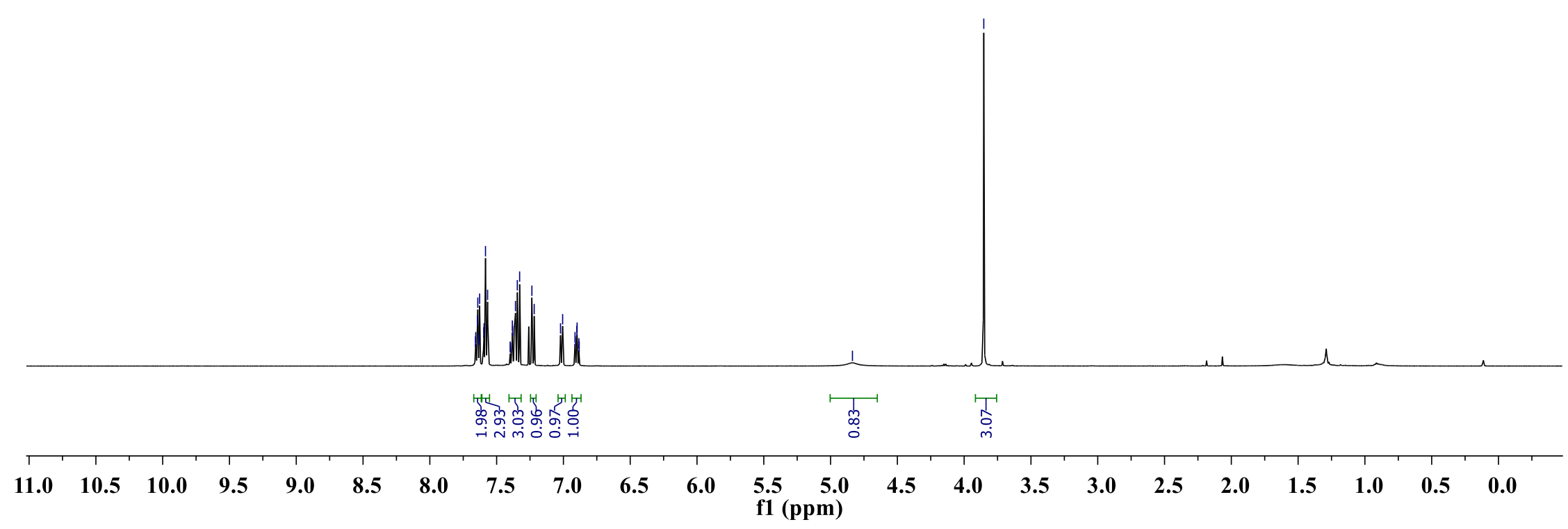




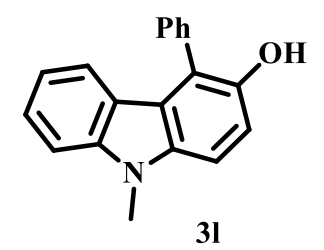

${ }^{13} \mathrm{C}$ NMR, $126 \mathrm{MHz}^{\mathrm{CDCl}}{ }_{3}$

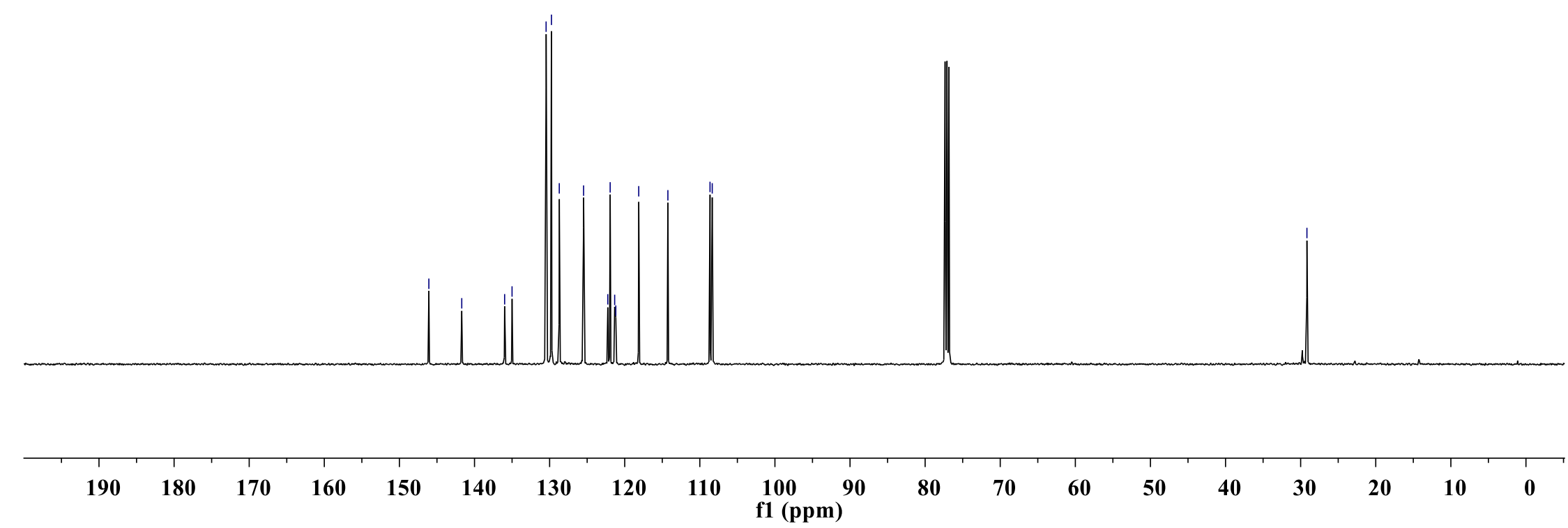




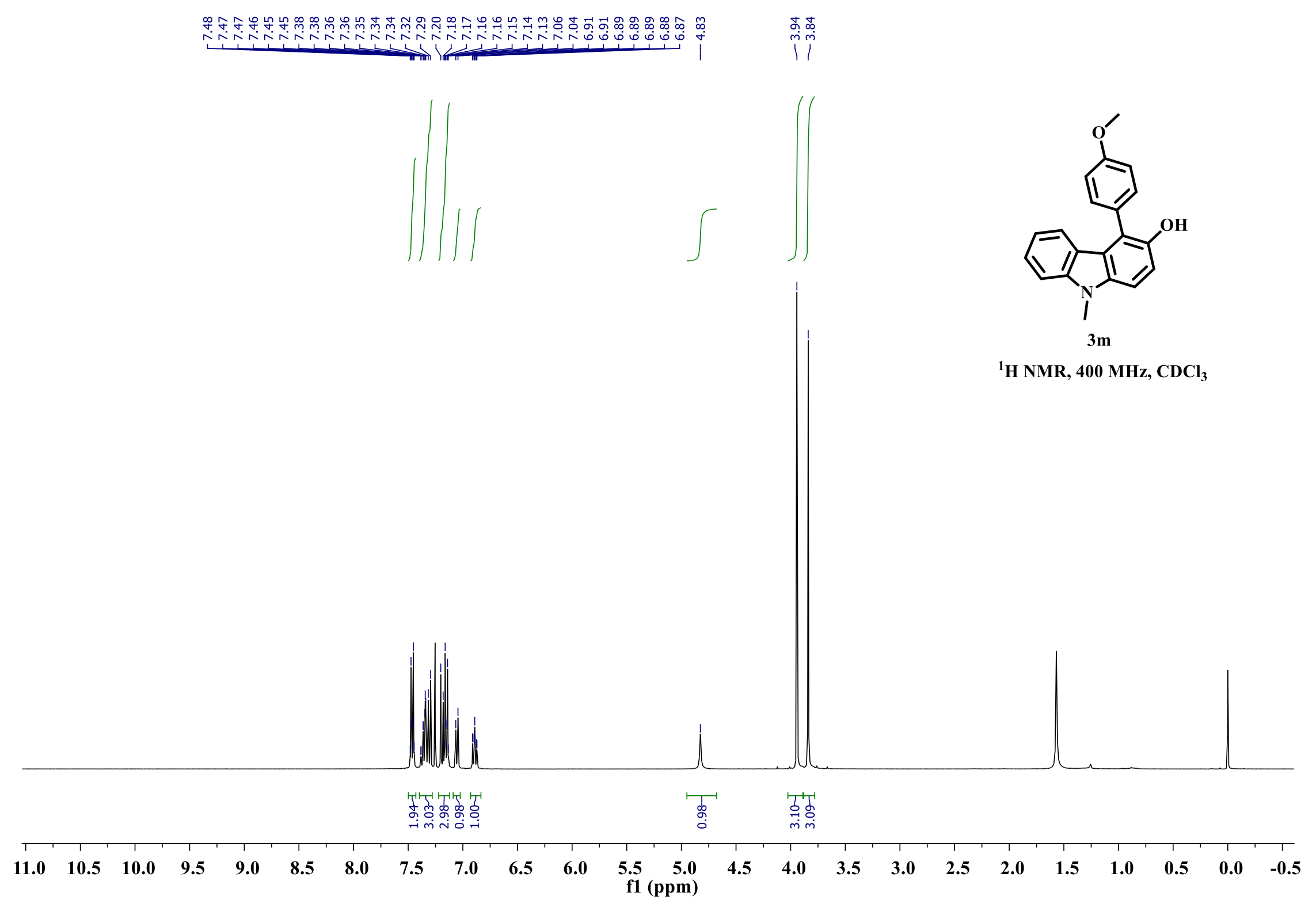




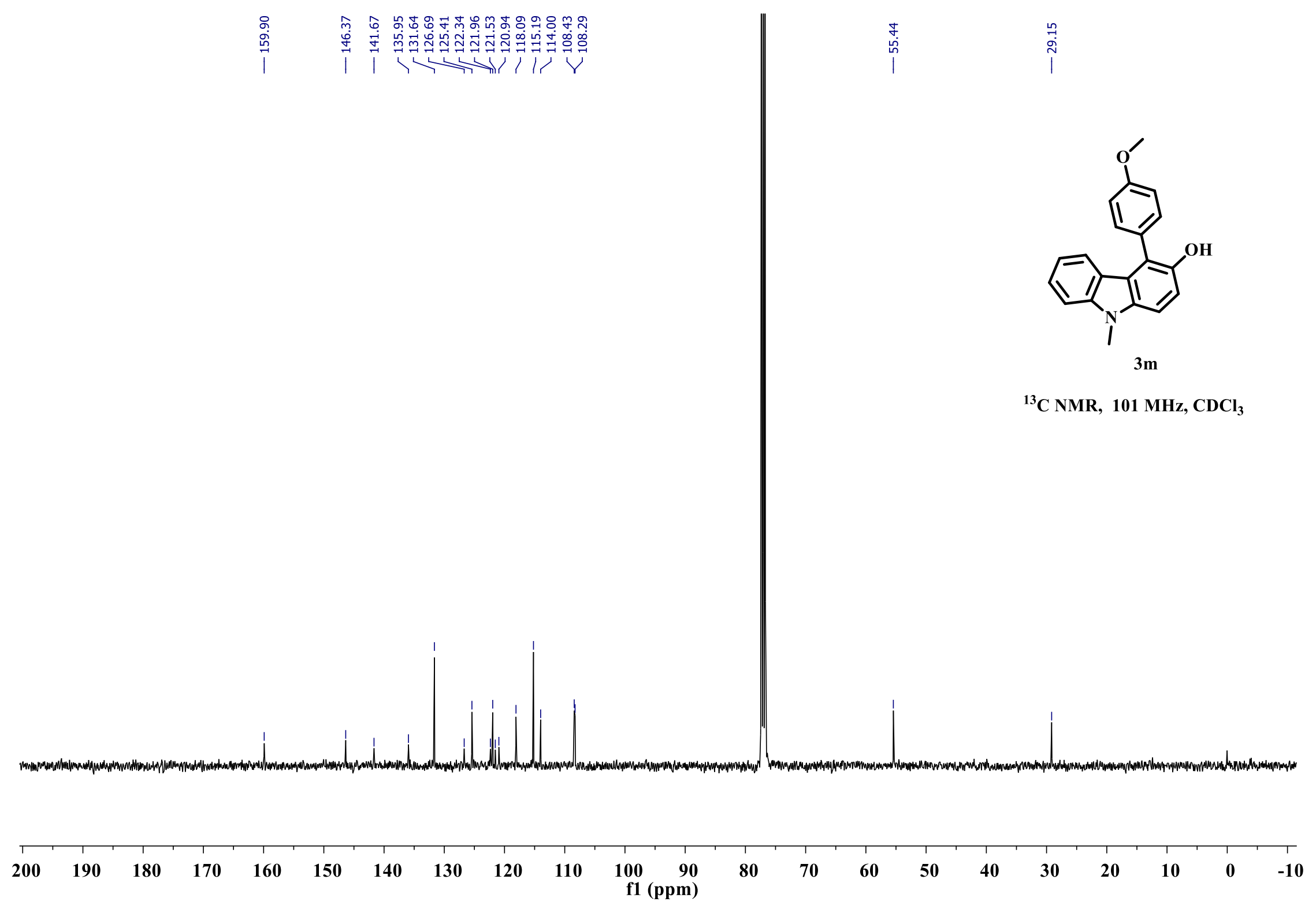




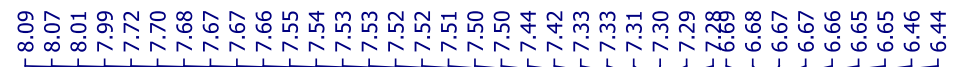

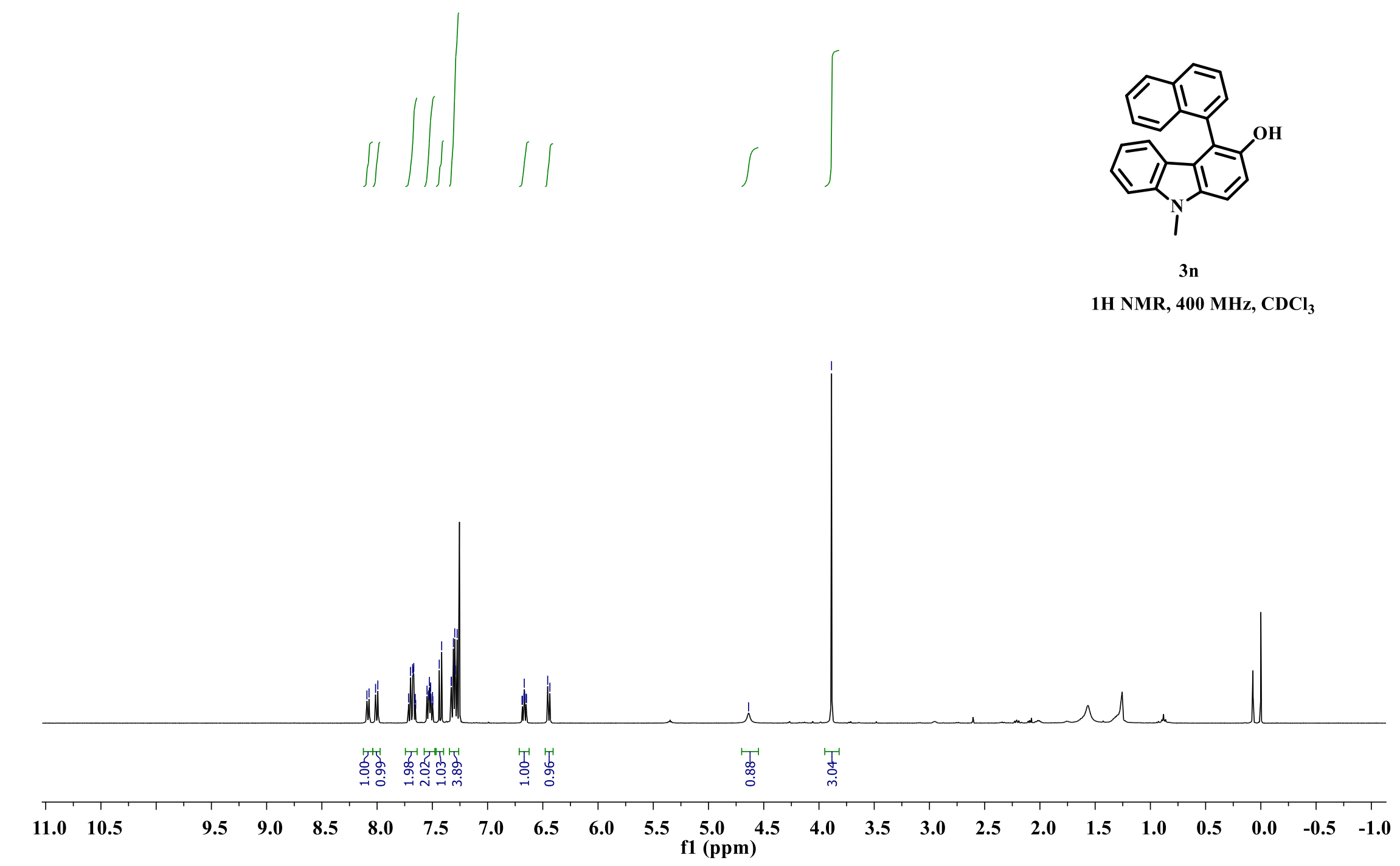



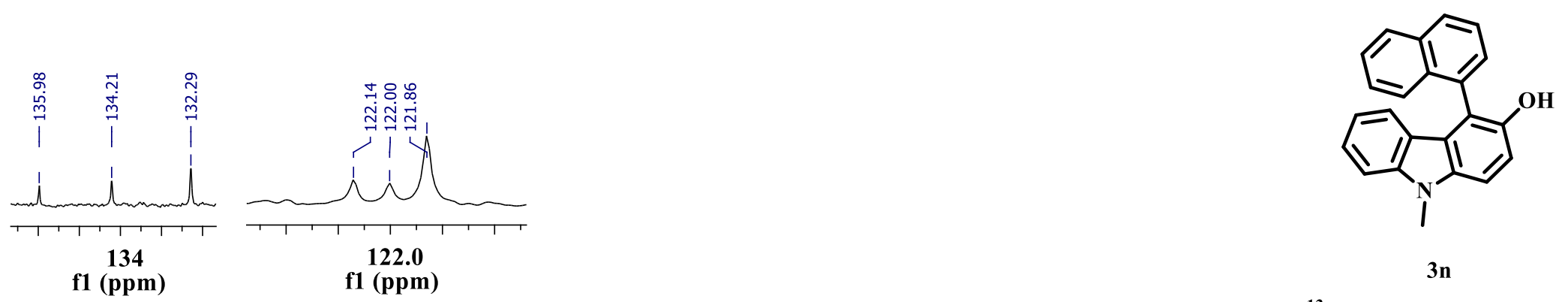

${ }^{13} \mathrm{C}$ NMR, $101 \mathrm{MHz}, \mathrm{CDCl}_{3}$

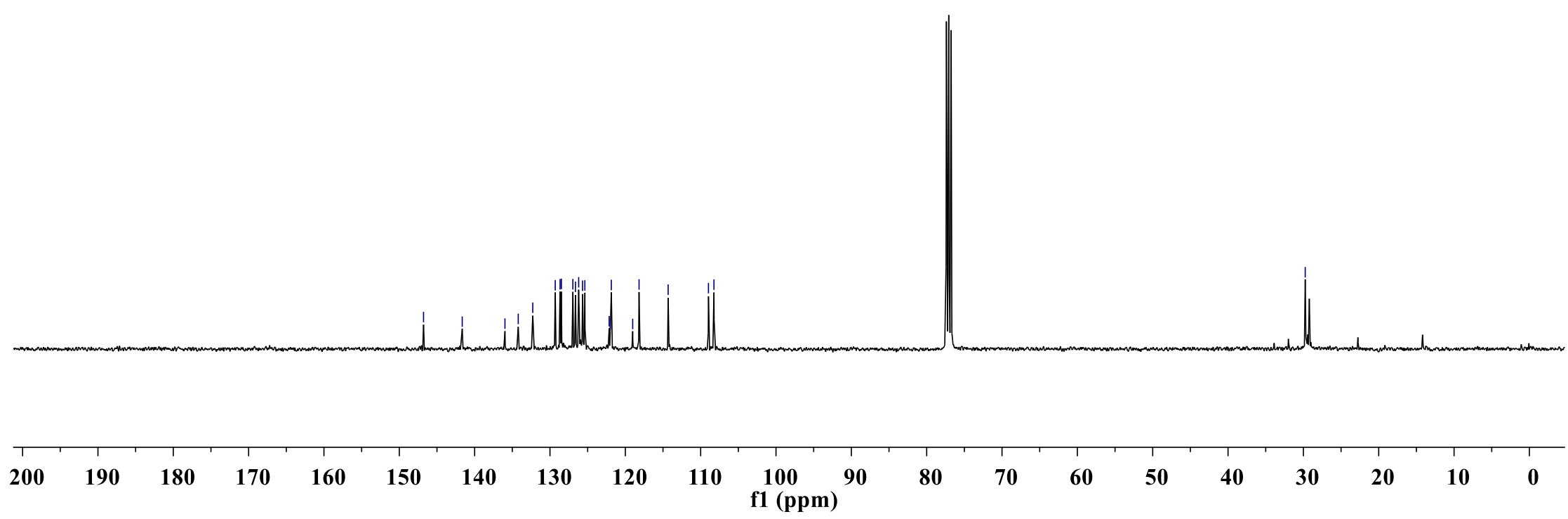



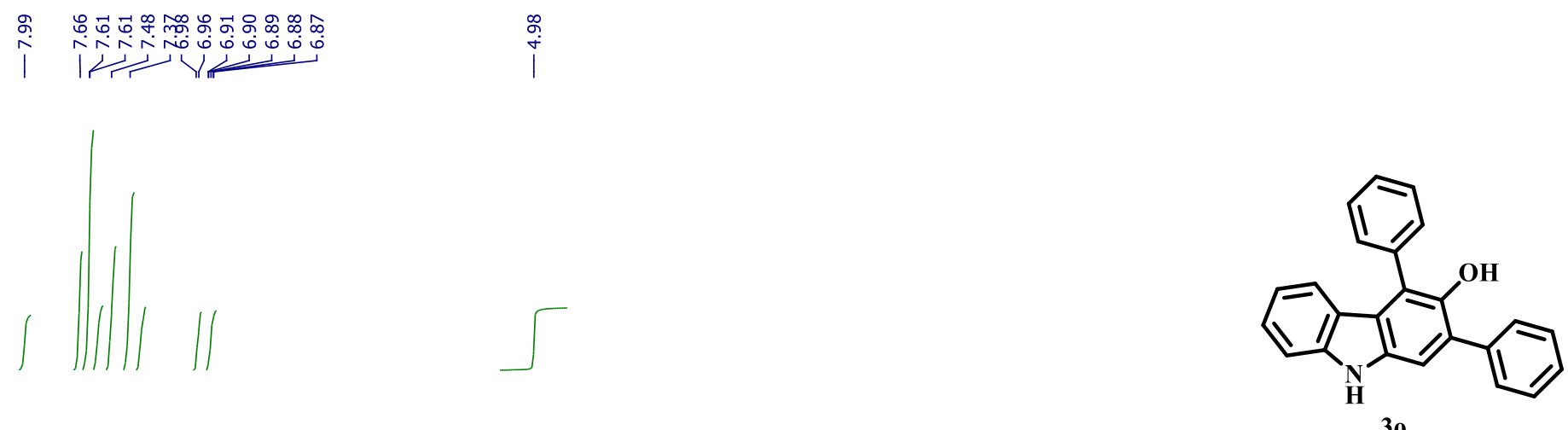

${ }^{1} \mathrm{H}$ NMR, $500 \mathrm{MHz}, \mathrm{CDCl}_{3}$

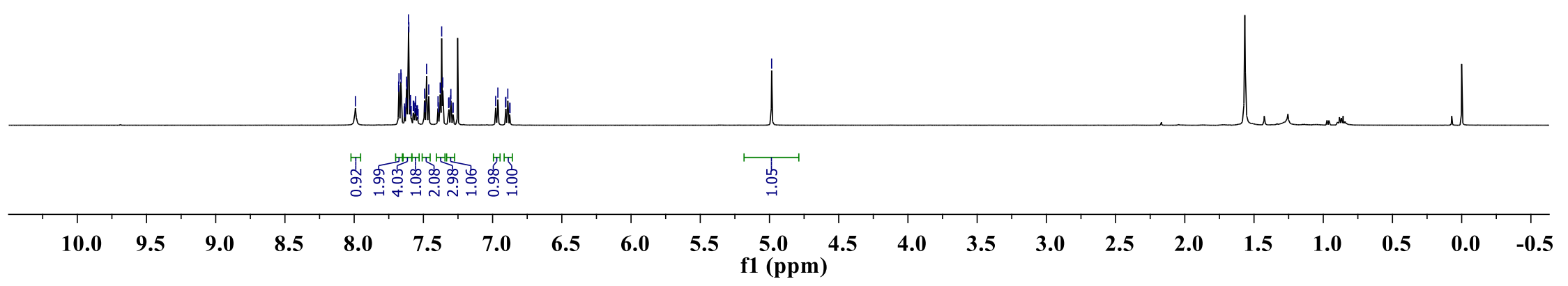




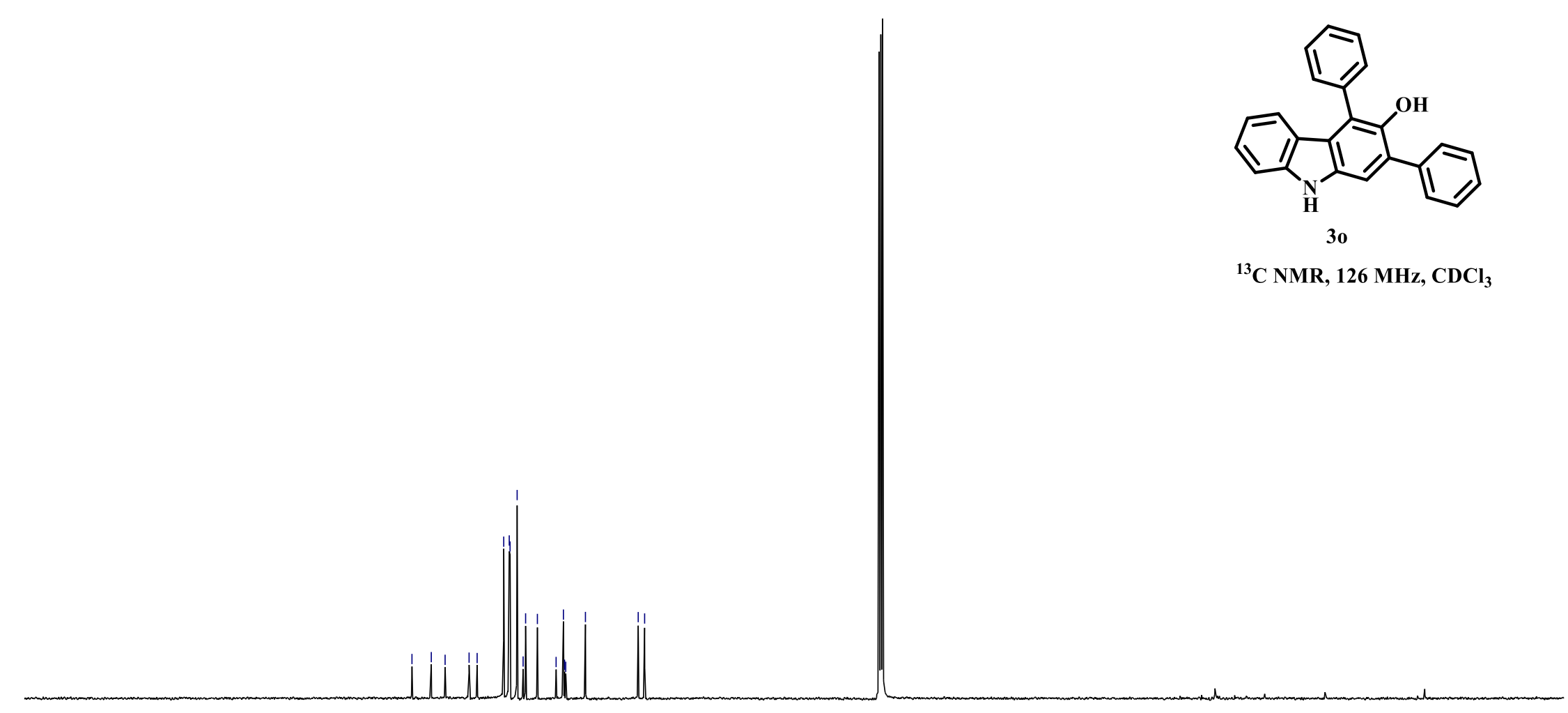

$$
\begin{array}{lllllllllll}
190 & 180 & 170 & 160 & 150 & 140 & 130 & 120 & 110 & 100 & \underset{\text { f1 }}{(\mathrm{ppm})}
\end{array}
$$


宓署

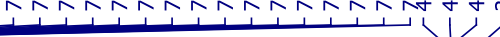

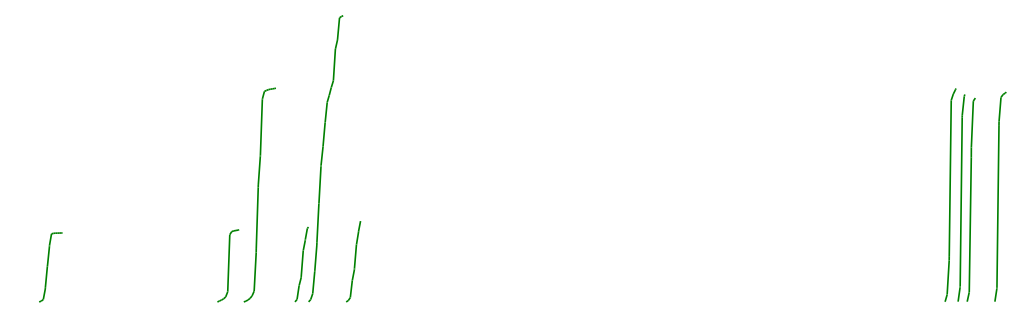

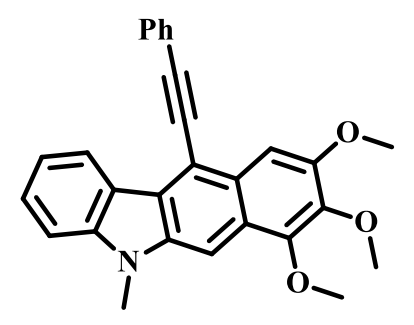

4a

${ }^{1} \mathrm{H}$ NMR, $400 \mathrm{MHz}, \mathrm{CDCl}_{3}$

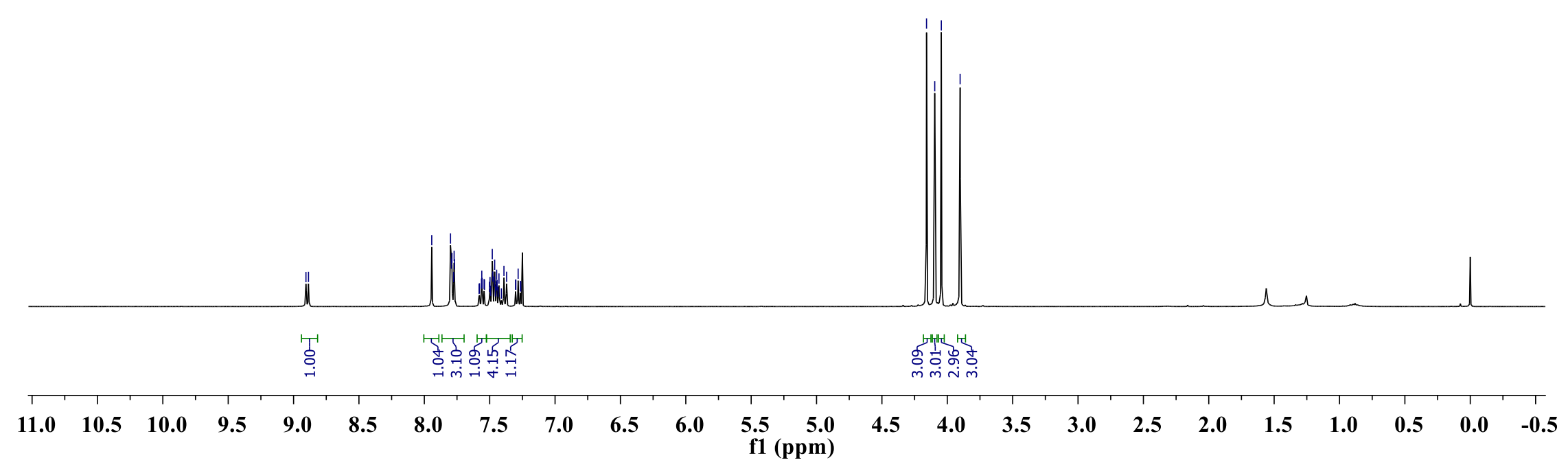




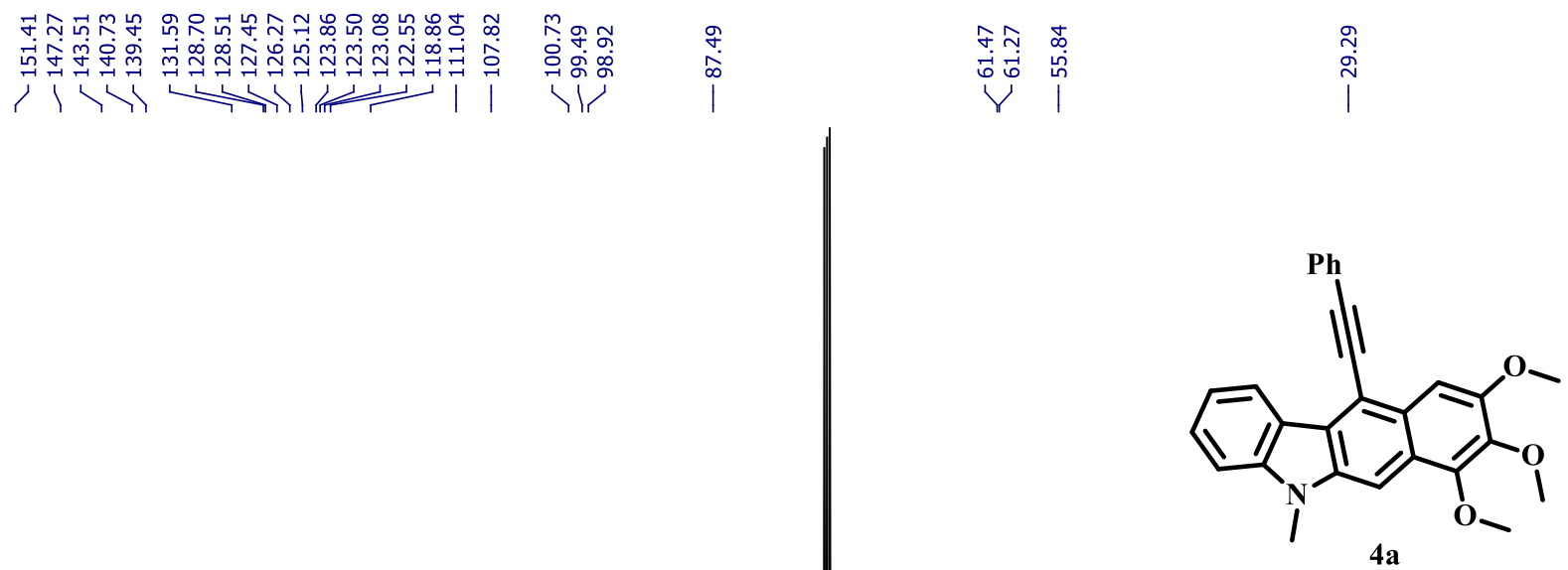

${ }^{13} \mathrm{C}$ NMR, $126 \mathrm{MHz}, \mathrm{CDCl}_{3}$
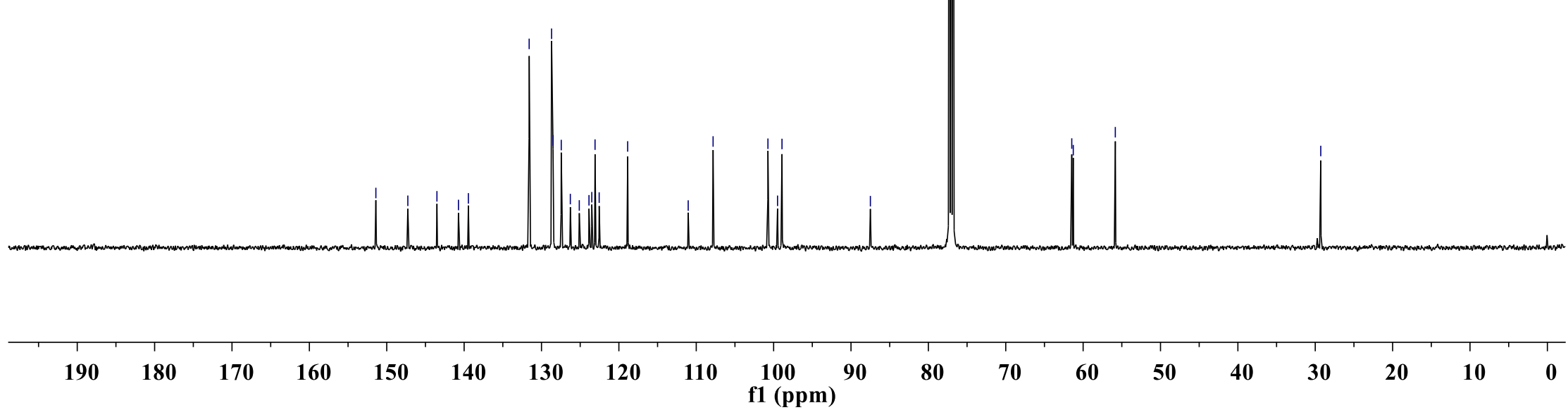

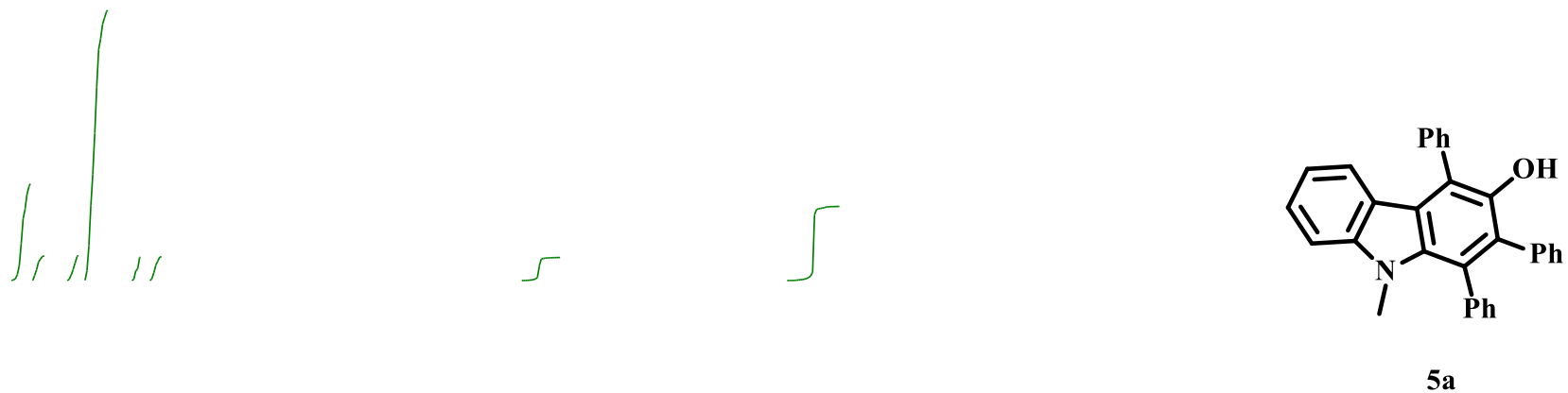

${ }^{1} \mathrm{H}_{\mathrm{NMR}}, 400 \mathrm{MHz}, \mathrm{CDCl}_{3}$

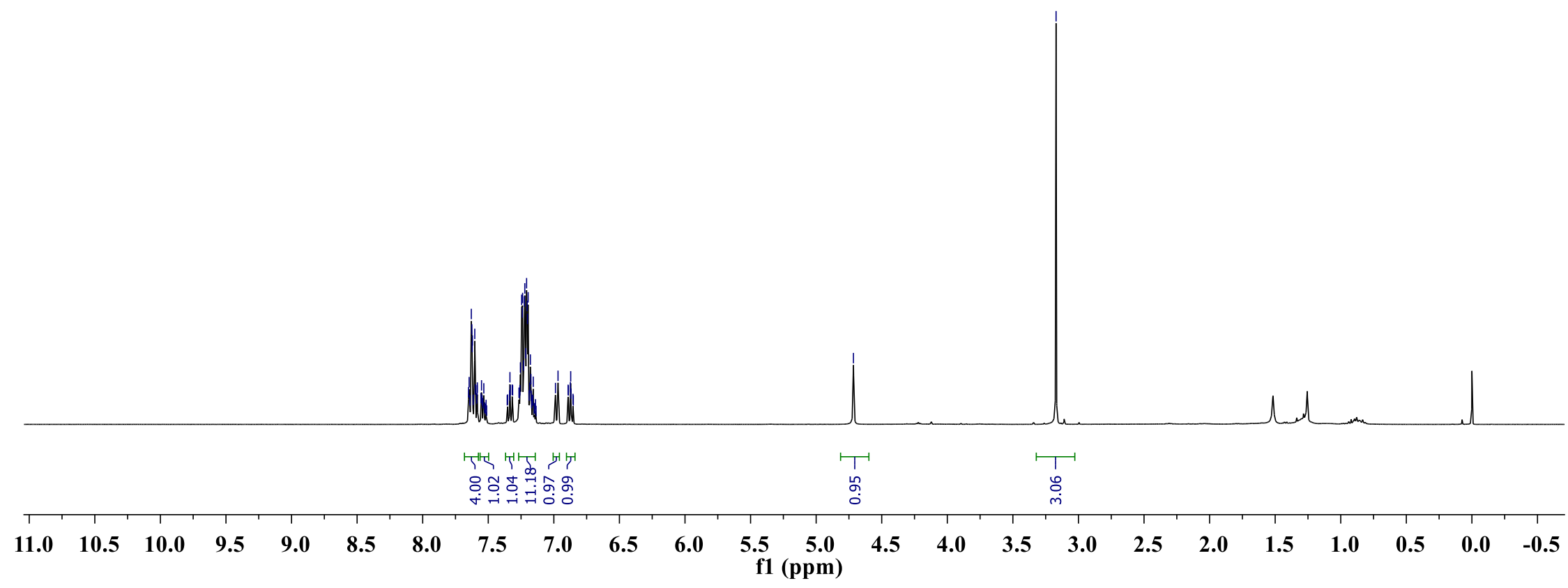



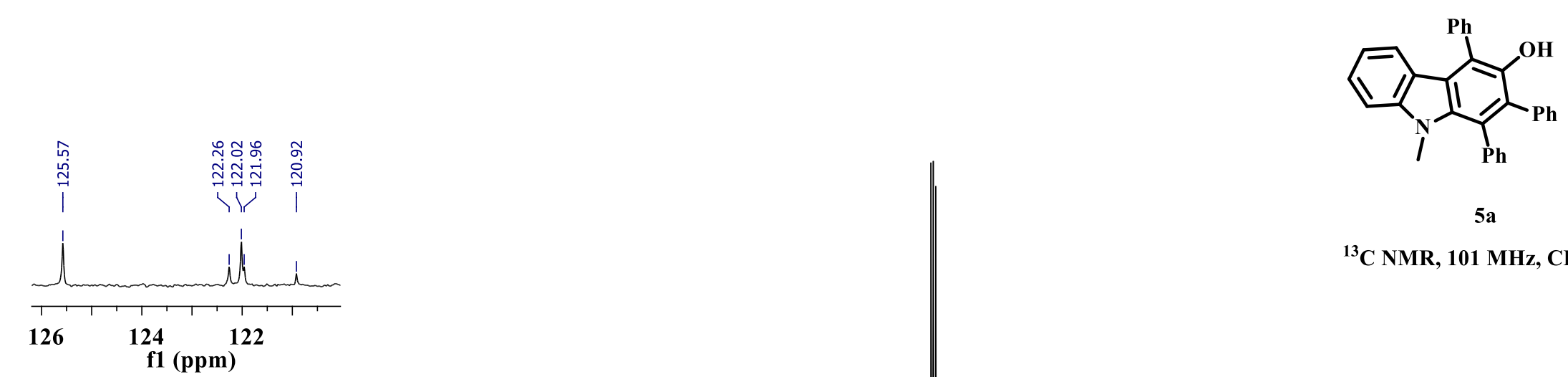

${ }^{13} \mathrm{C}$ NMR, $101 \mathrm{MHz}, \mathrm{CDCl}_{3}$

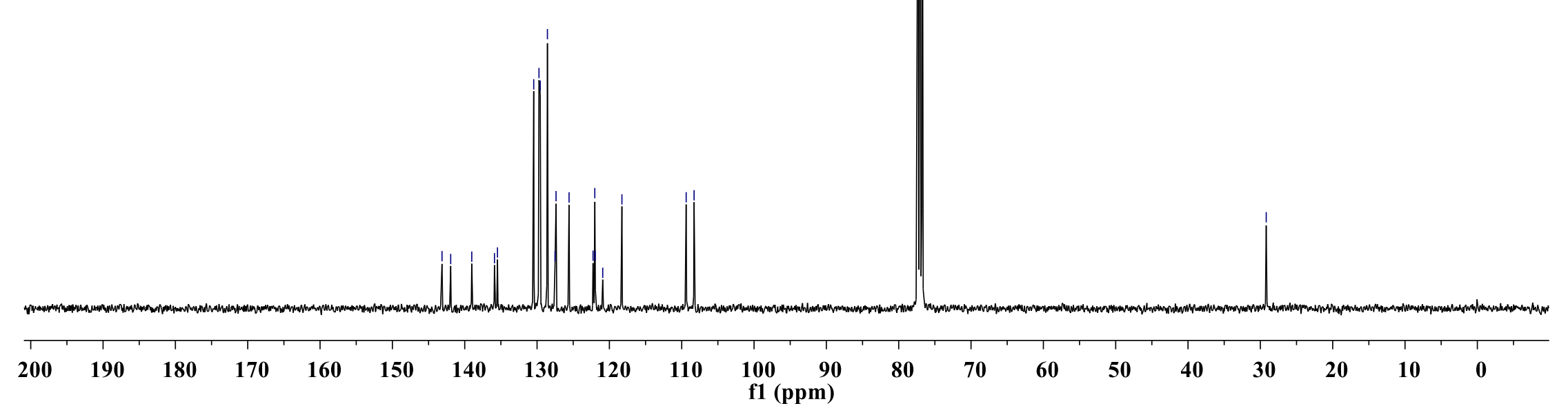




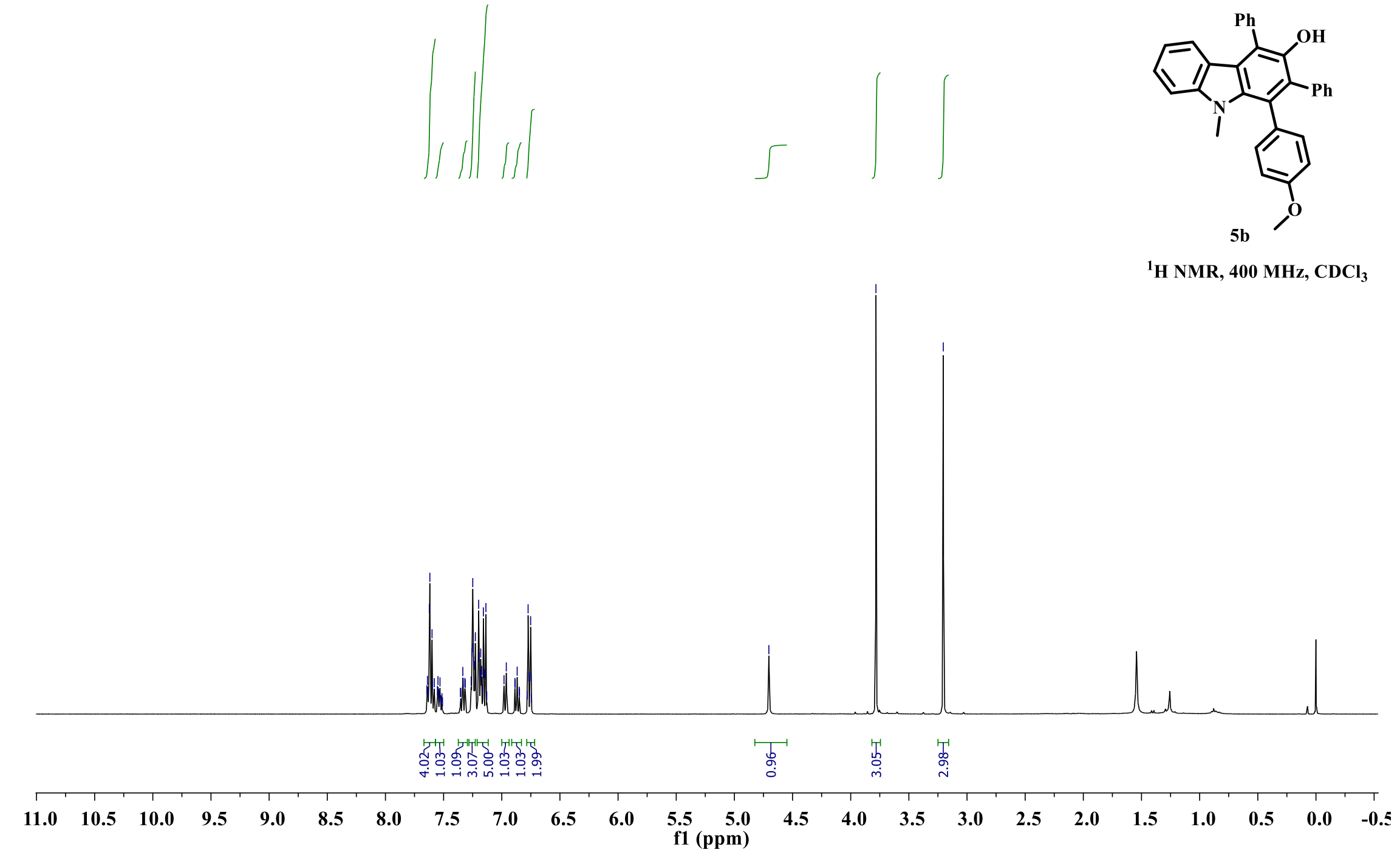




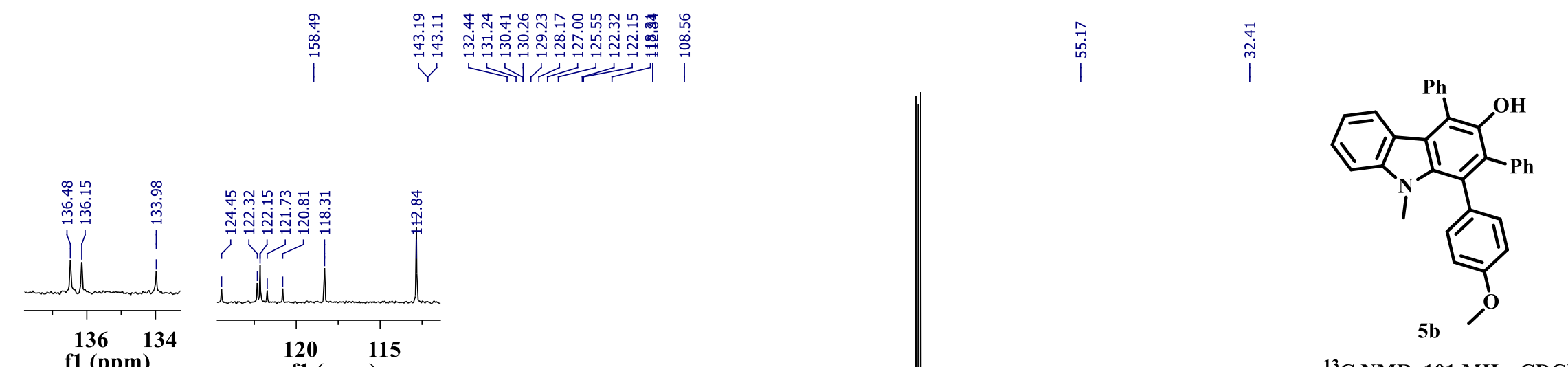

${ }^{13} \mathrm{C}$ NMR, $101 \mathrm{MHz}, \mathrm{CDCl}_{3}$

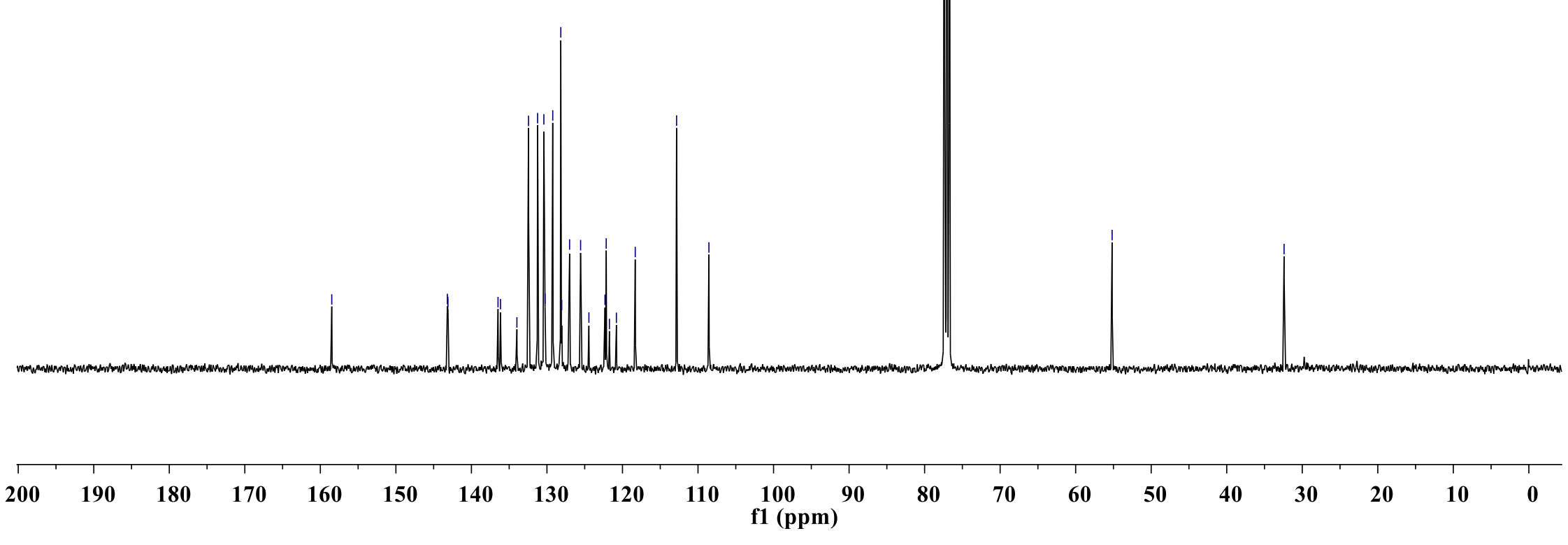




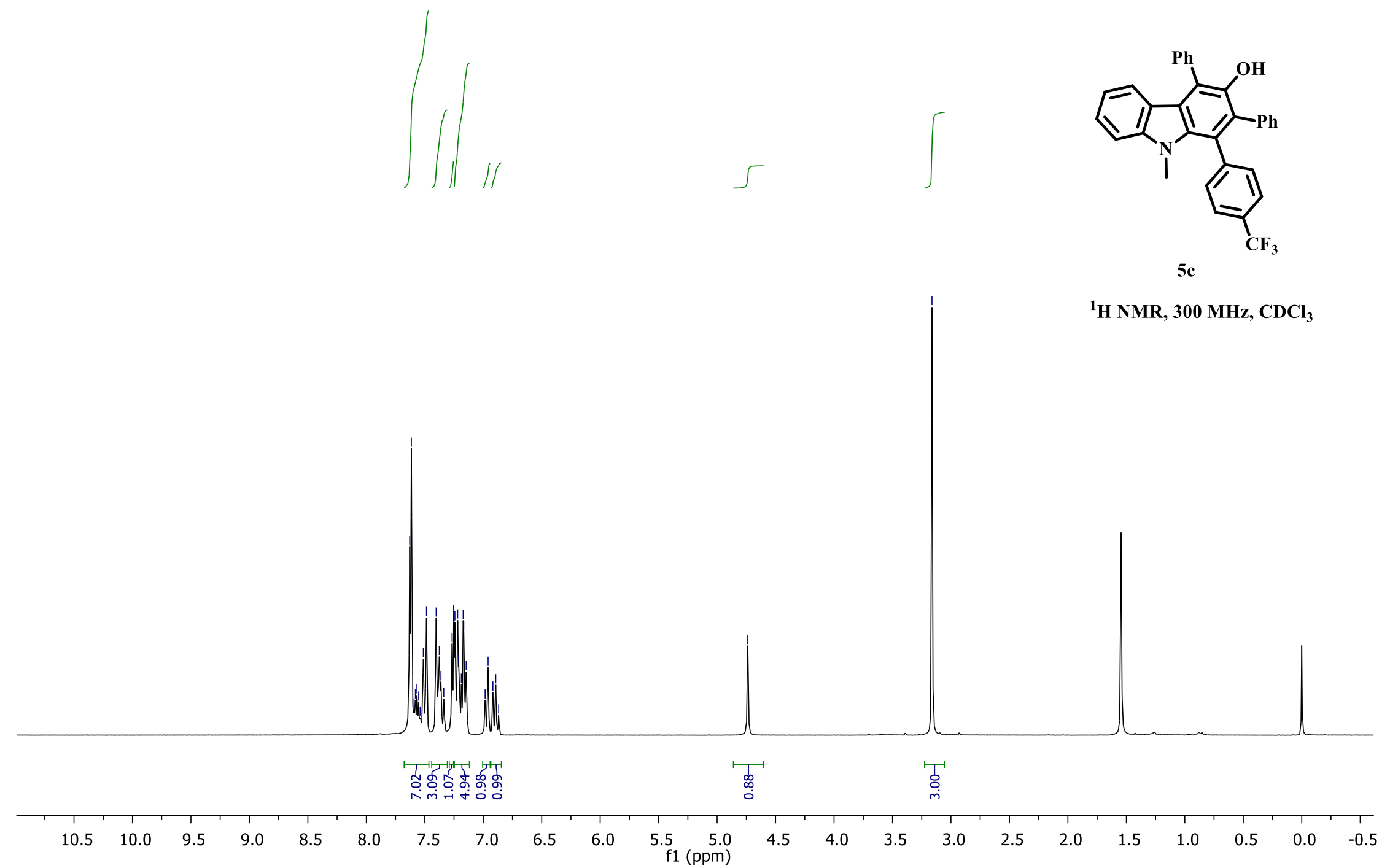


DATTHA-M0009
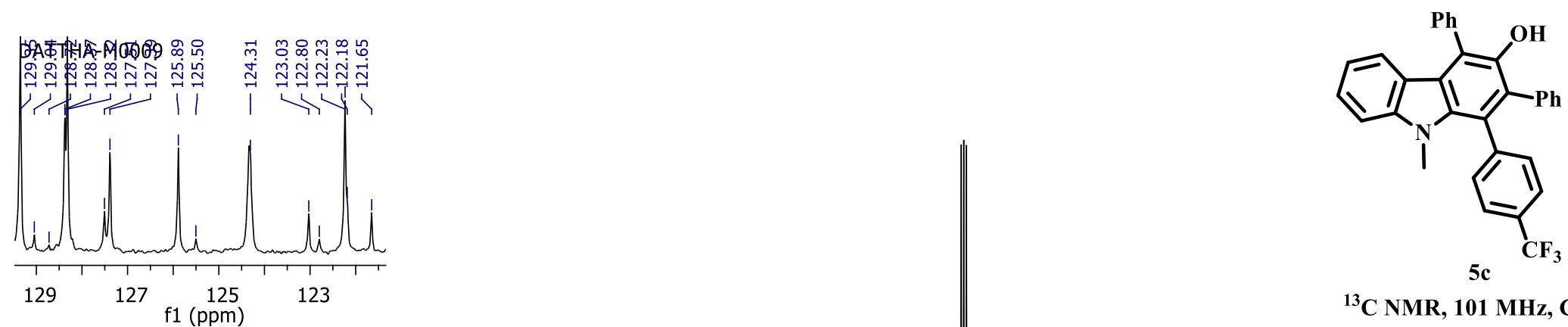

${ }^{13} \mathrm{C}$ NMR, $101 \mathrm{MHz}, \mathrm{CDCl}_{3}$

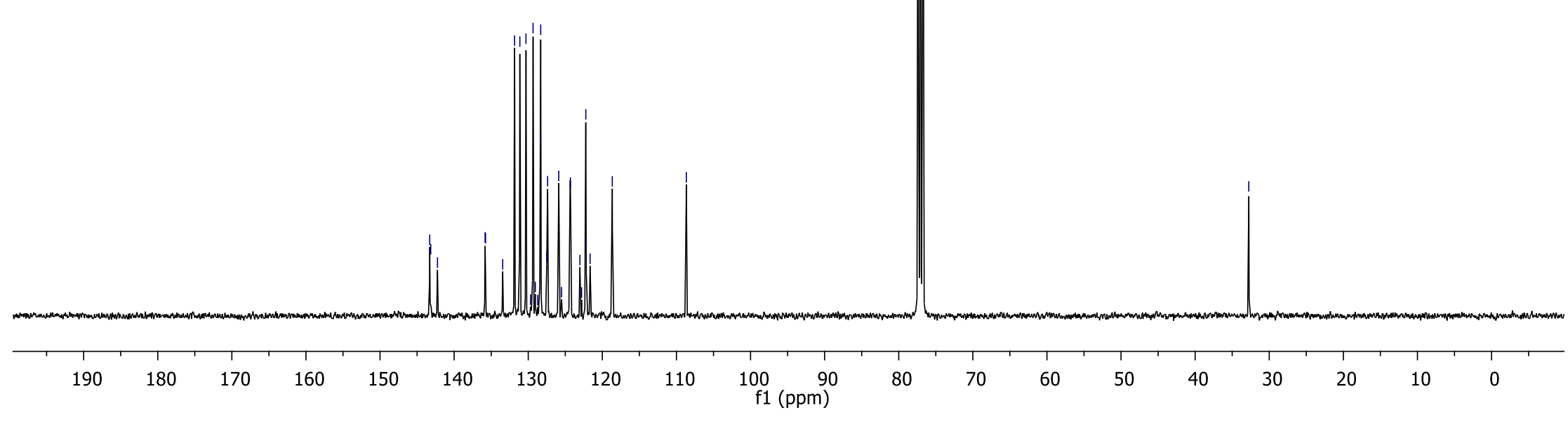



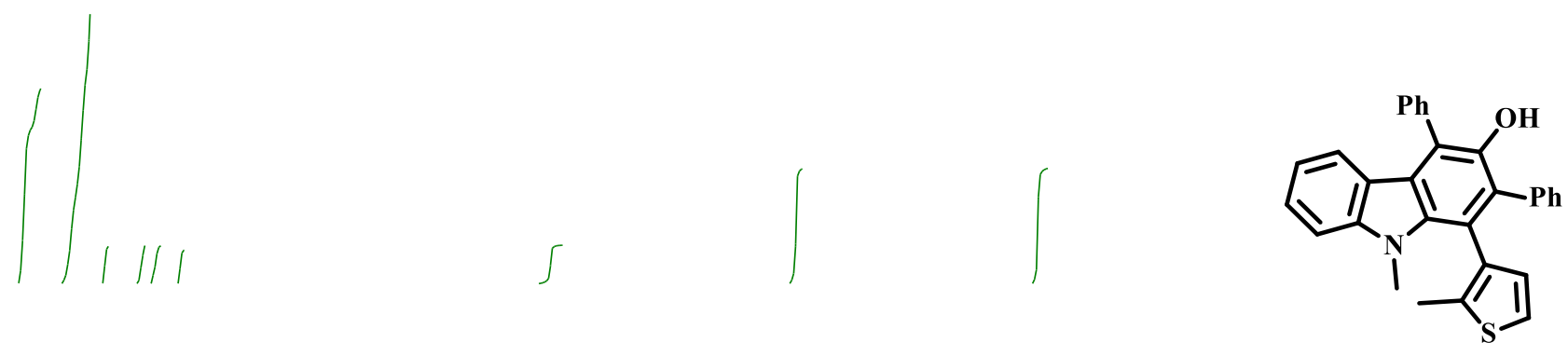

$5 d$

${ }^{1} \mathrm{H}$ NMR, $500 \mathrm{MHz}, \mathrm{CDCl}_{3}$

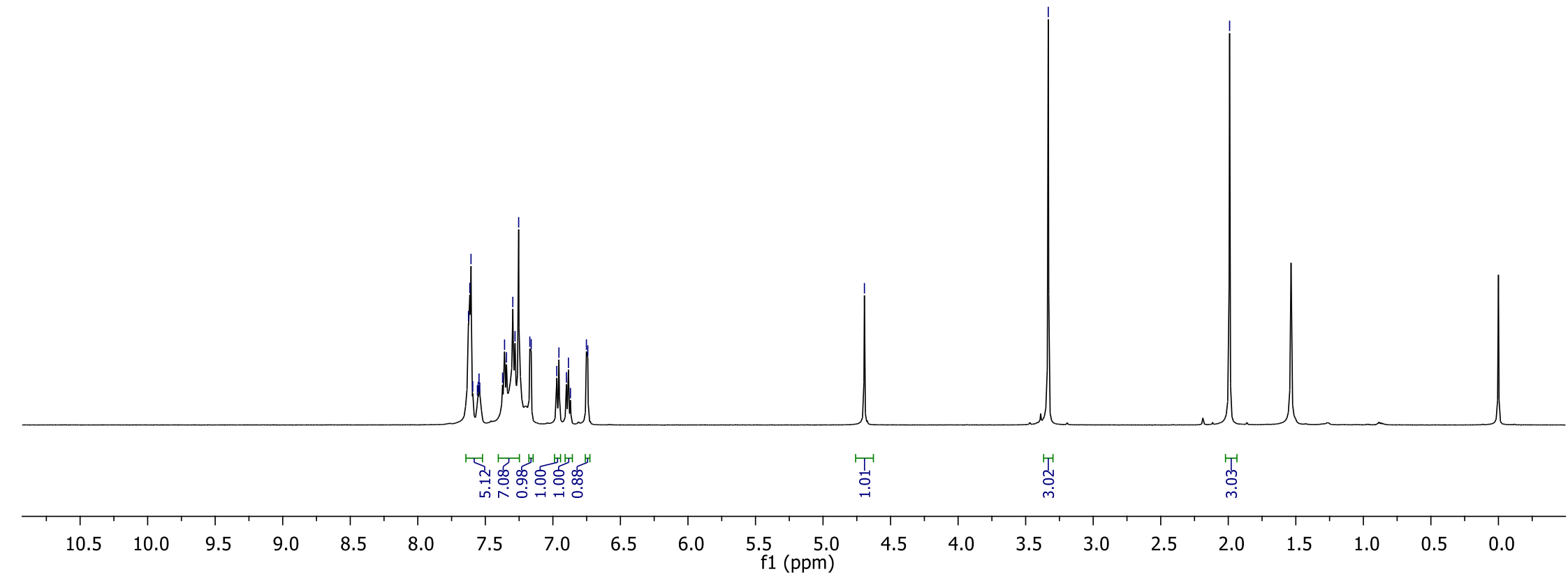


DATTHA-M0009

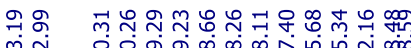

V

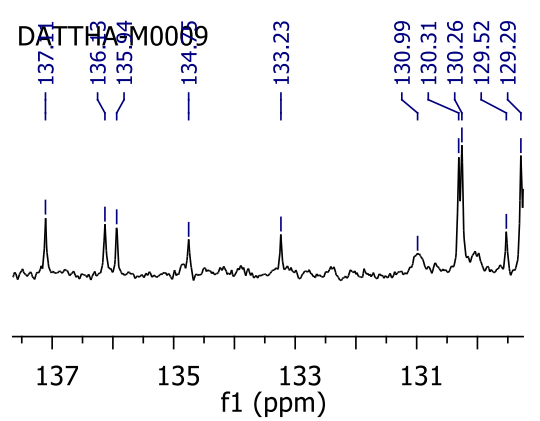

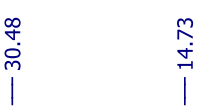

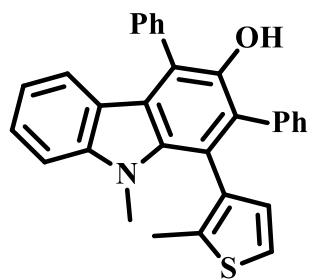

${ }^{13} \mathrm{C}$ NMR, $101 \mathrm{MHz}, \mathrm{CDCl}_{3}$

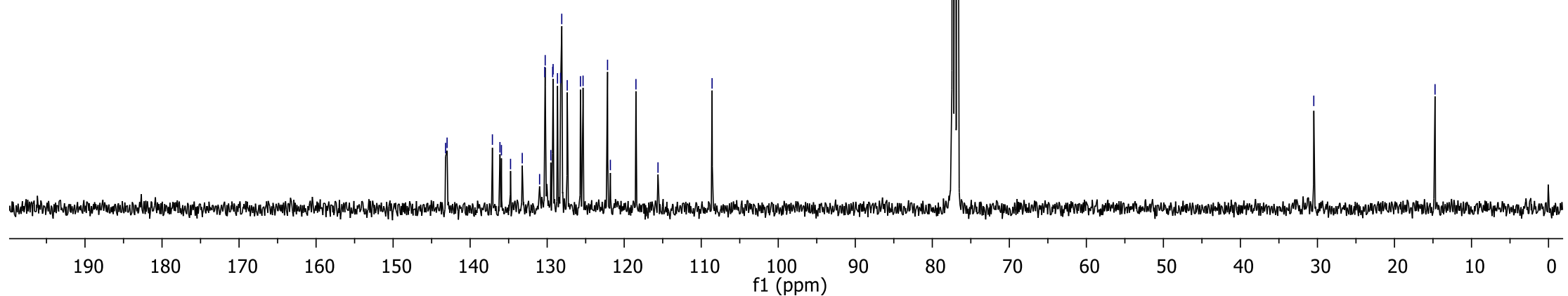



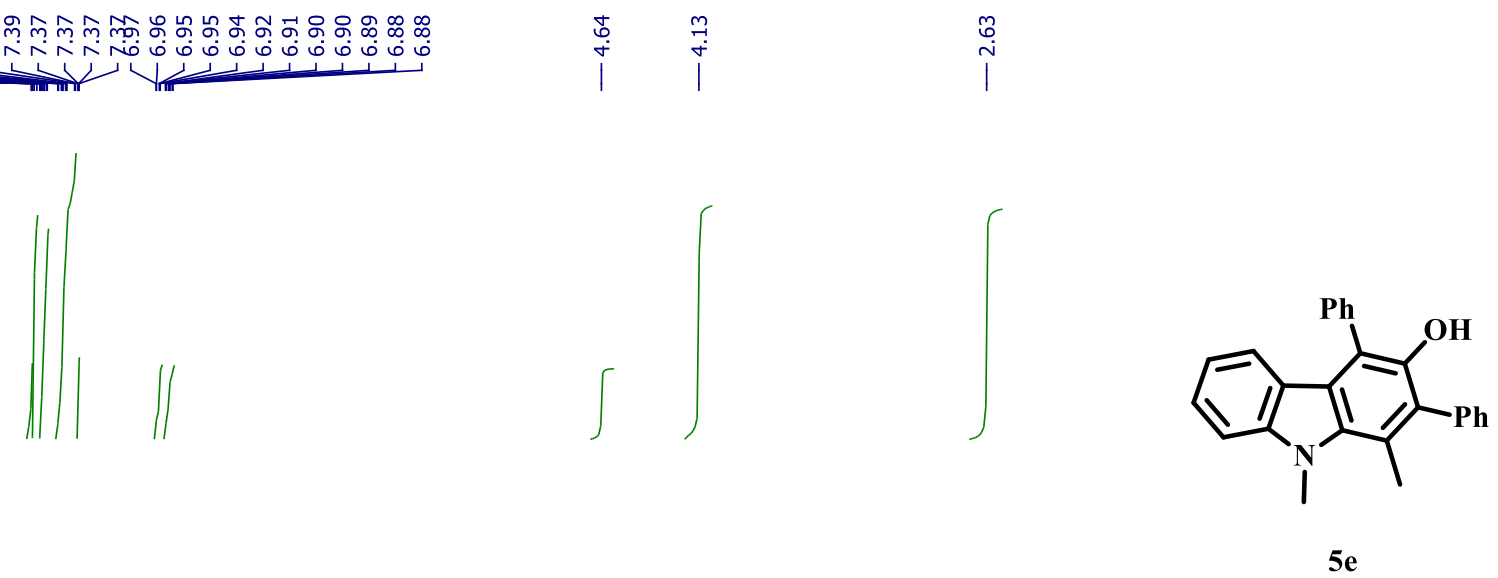

${ }^{1} \mathrm{H}$ NMR , $400 \mathrm{MHz}, \mathrm{CDCl}_{3}$

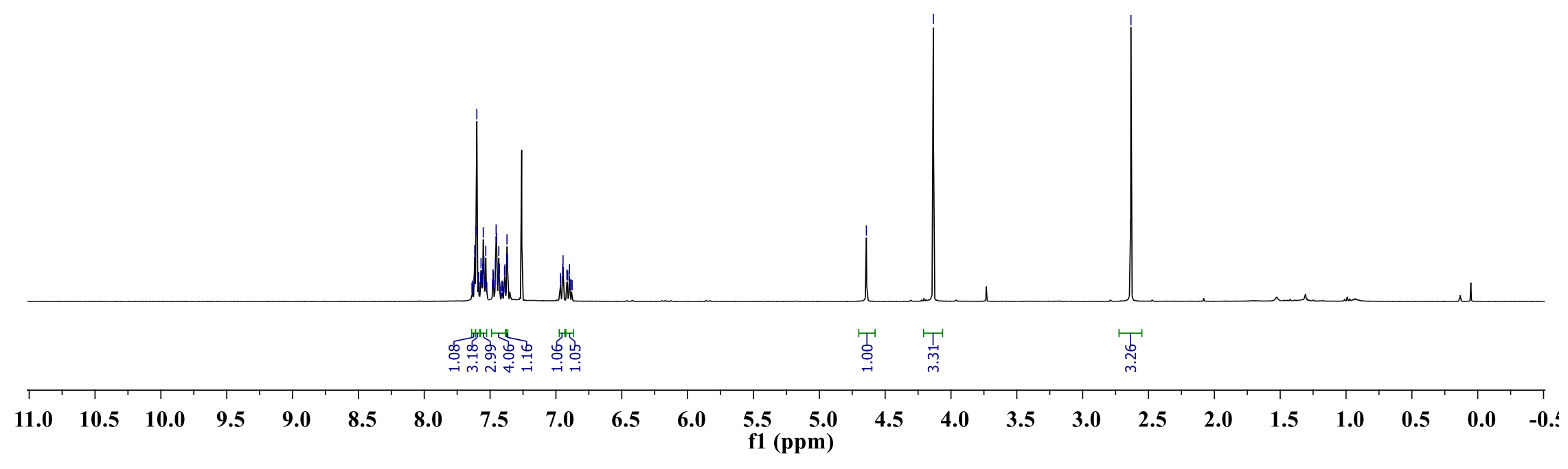




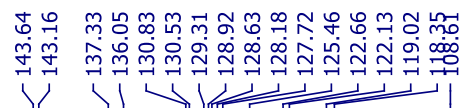

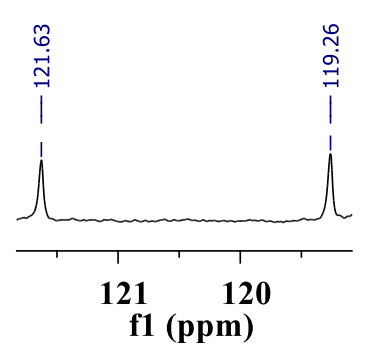

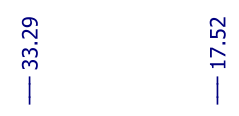

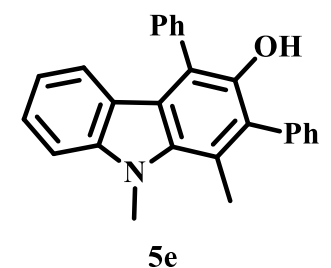

${ }^{13} \mathrm{C}$ NMR, $126 \mathrm{MHz}, \mathrm{CDCl}_{3}$
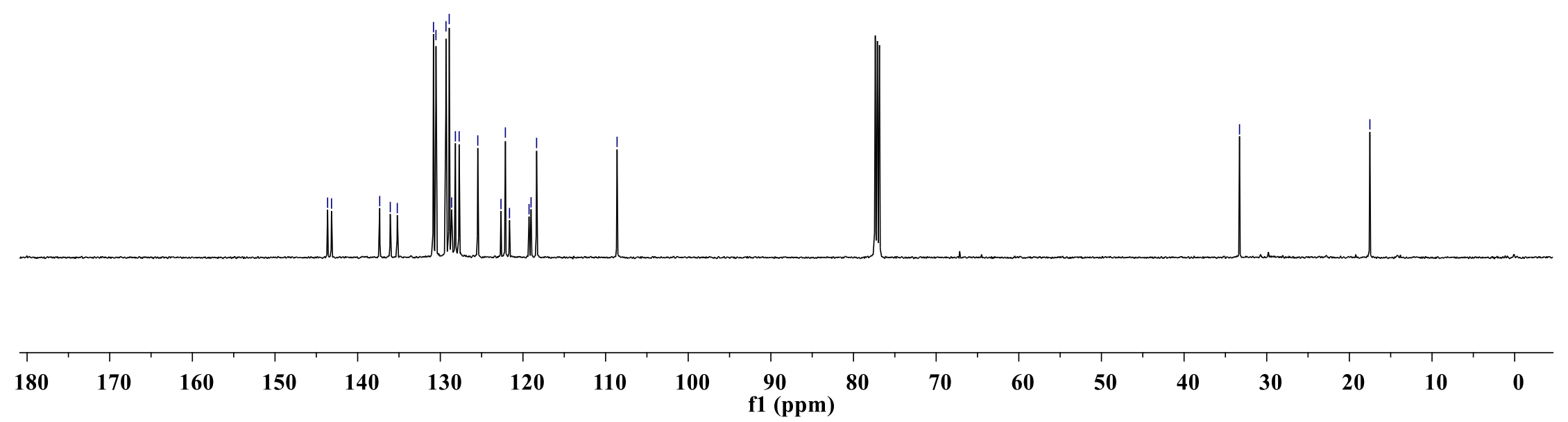


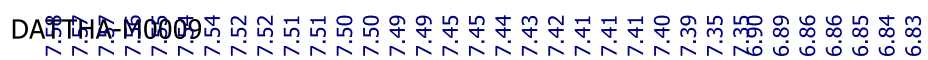
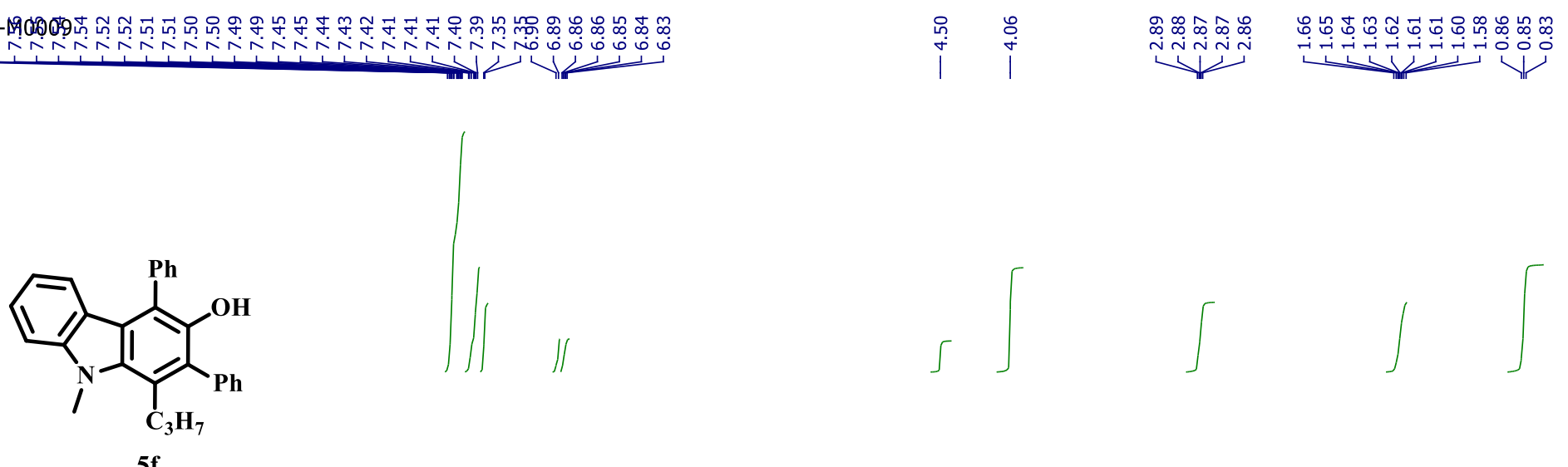

${ }^{1} \mathrm{H}$ NMR, $500 \mathrm{MHz}, \mathrm{CDCl}_{3}$

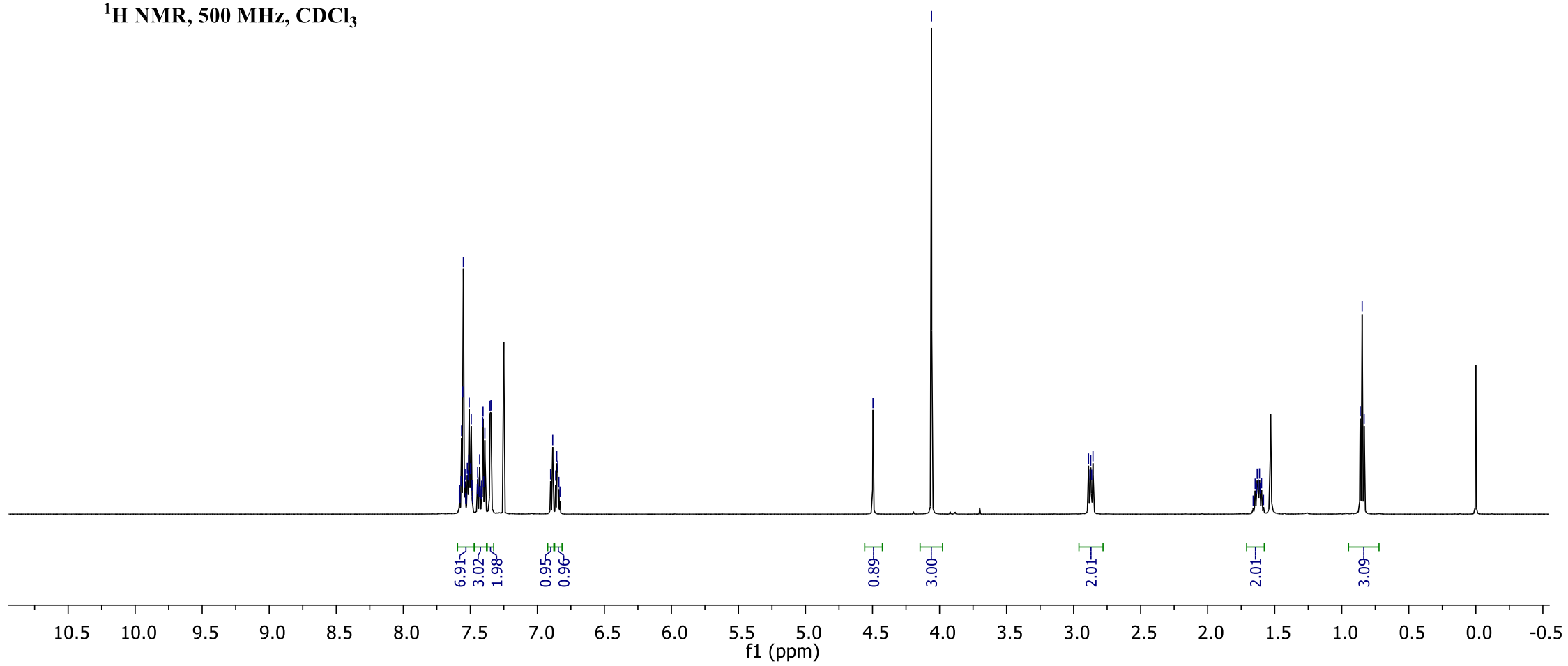


DATTA-M0009

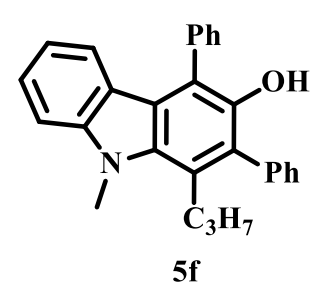

${ }^{13}$ C NMR, 126 MHz, CDCl
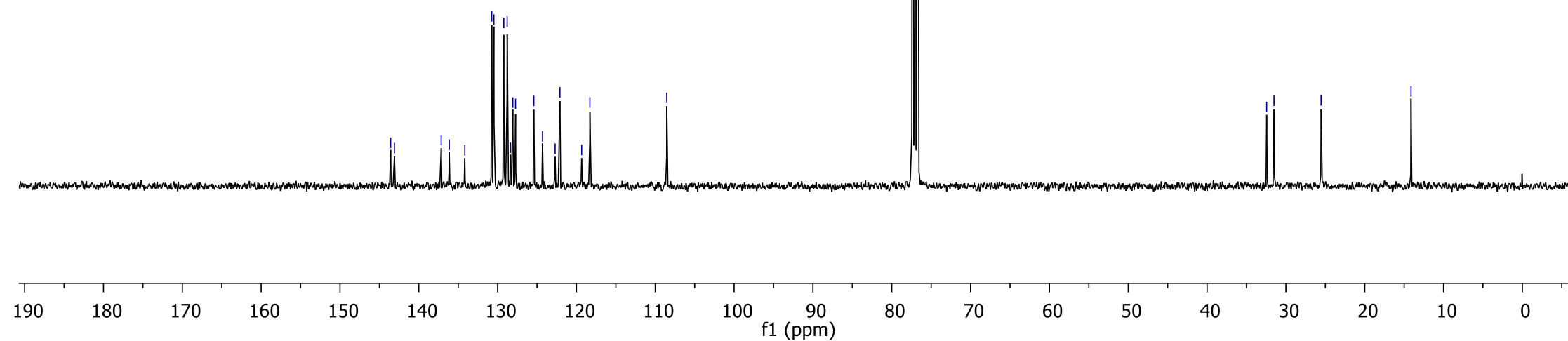

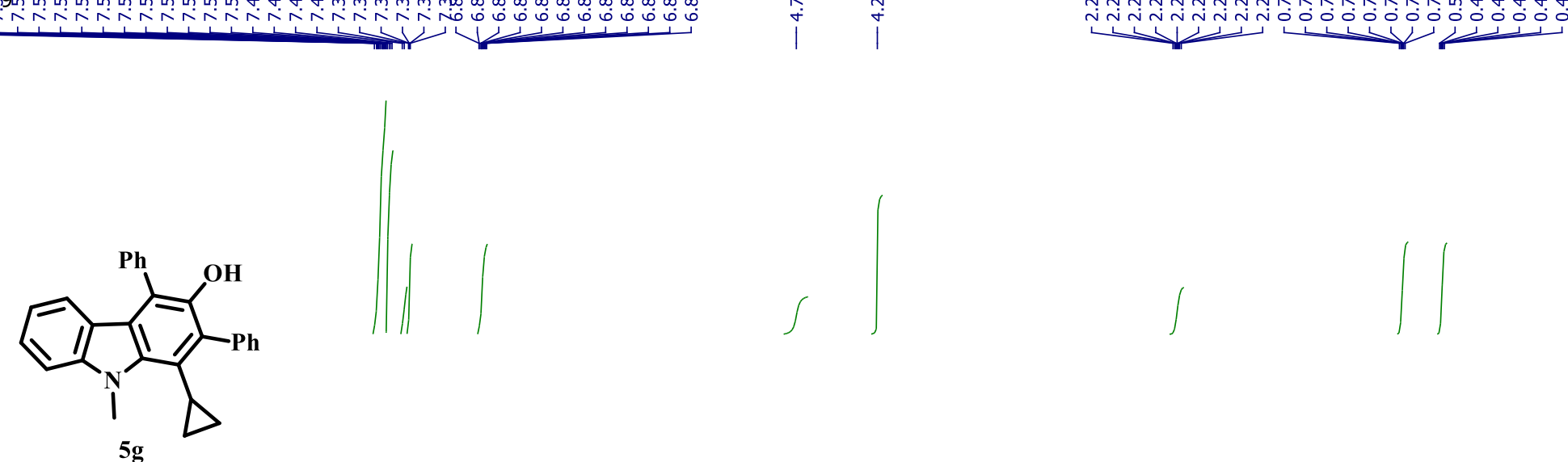

${ }^{1} \mathrm{H}$ NMR, $500 \mathrm{MHz}, \mathrm{CDCl}_{3}$

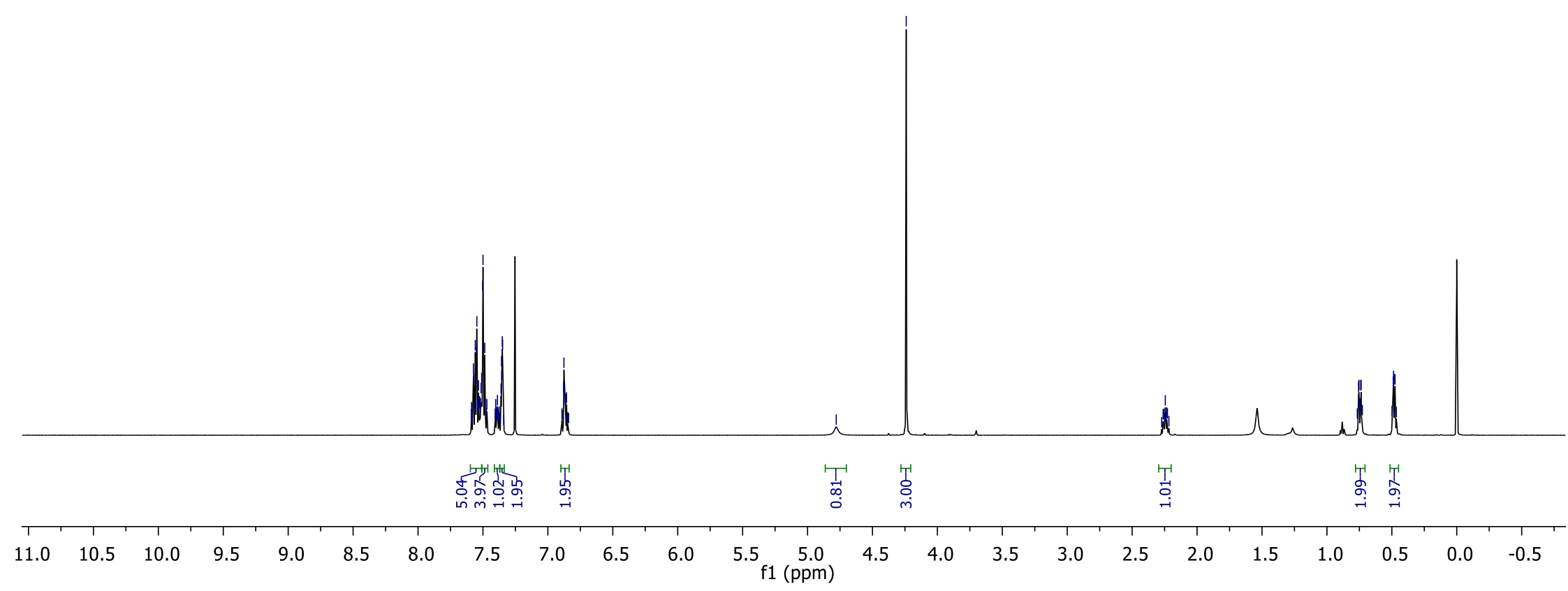


ROSHAN-M0009

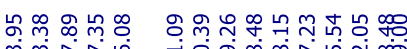

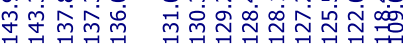

广र
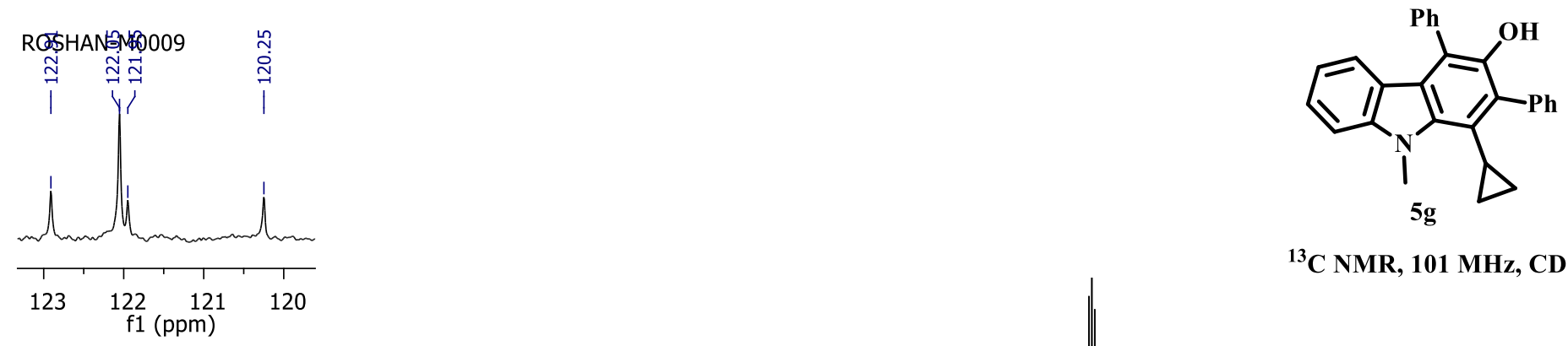

${ }^{13} \mathrm{C}$ NMR, $101 \mathrm{MHz}, \mathrm{CDCl}_{3}$

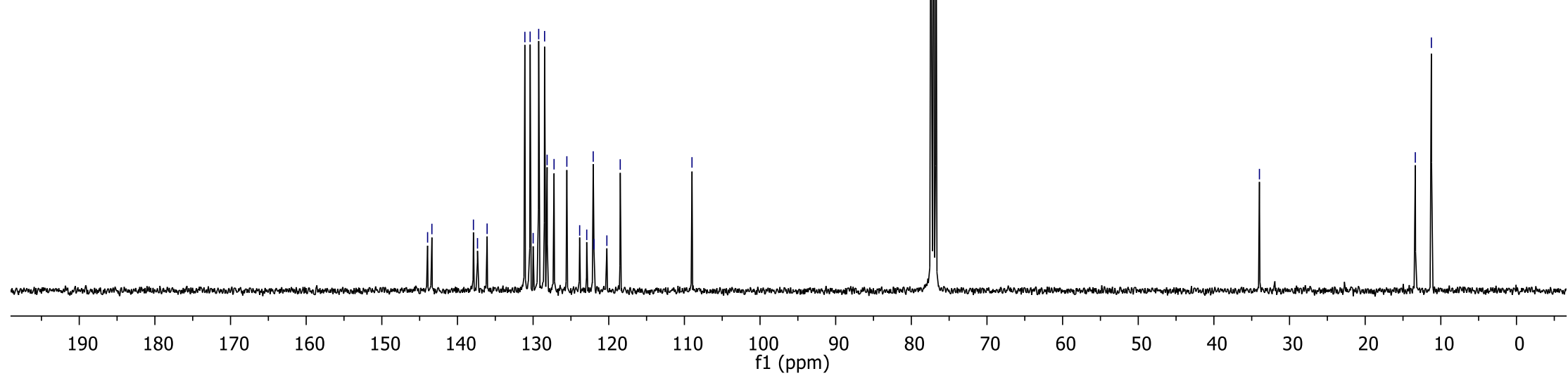




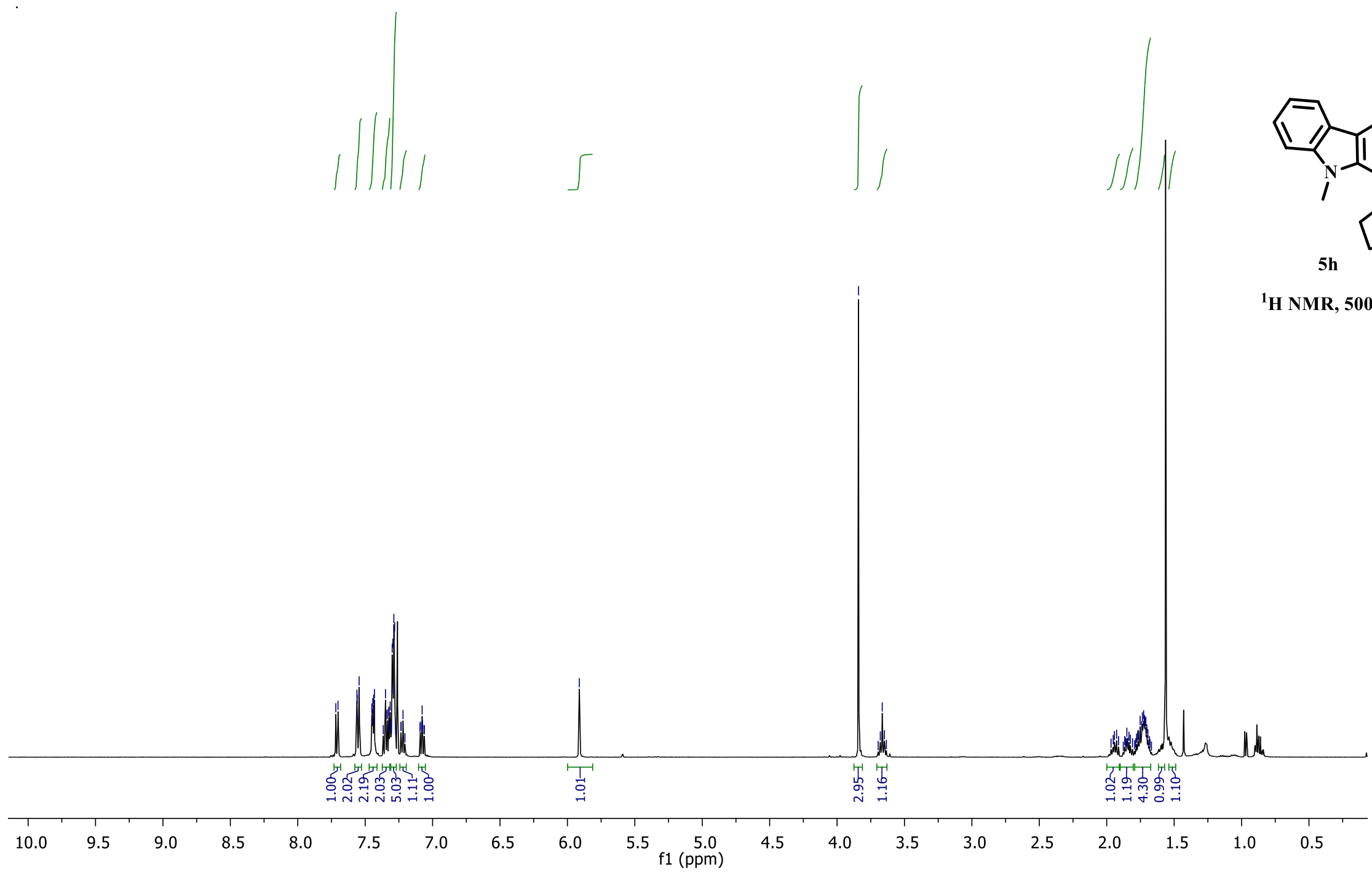


DAFT席 190009

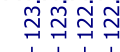

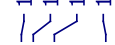

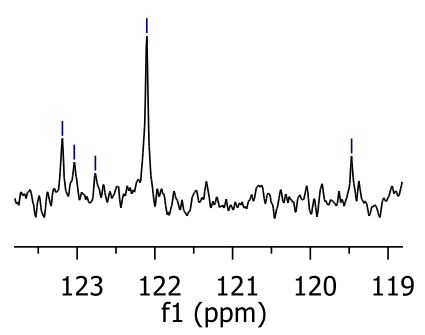

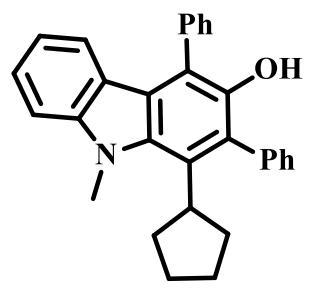

$5 \mathrm{~h}$

${ }^{13} \mathrm{C}$ NMR, $101 \mathrm{MHz}, \mathrm{CDCl}_{3}$

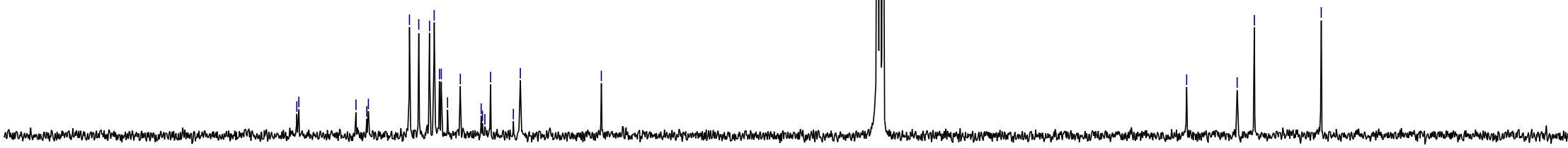

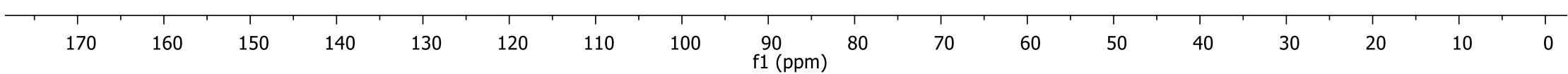



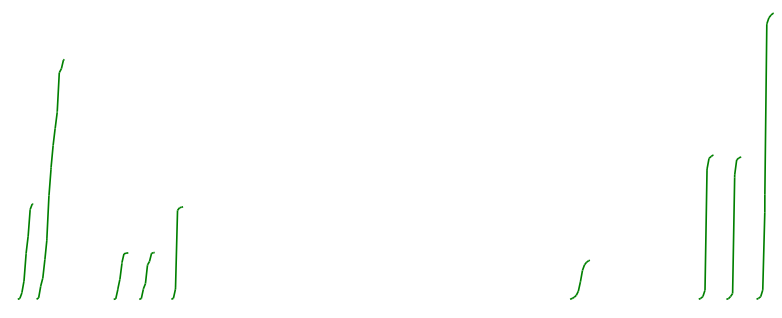

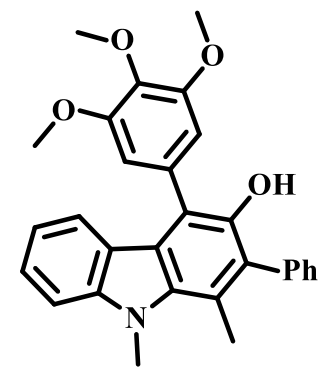

$5 i$

${ }^{1} \mathrm{H}$ NMR, $400 \mathrm{MHz}, \mathrm{CDCl}_{3}$

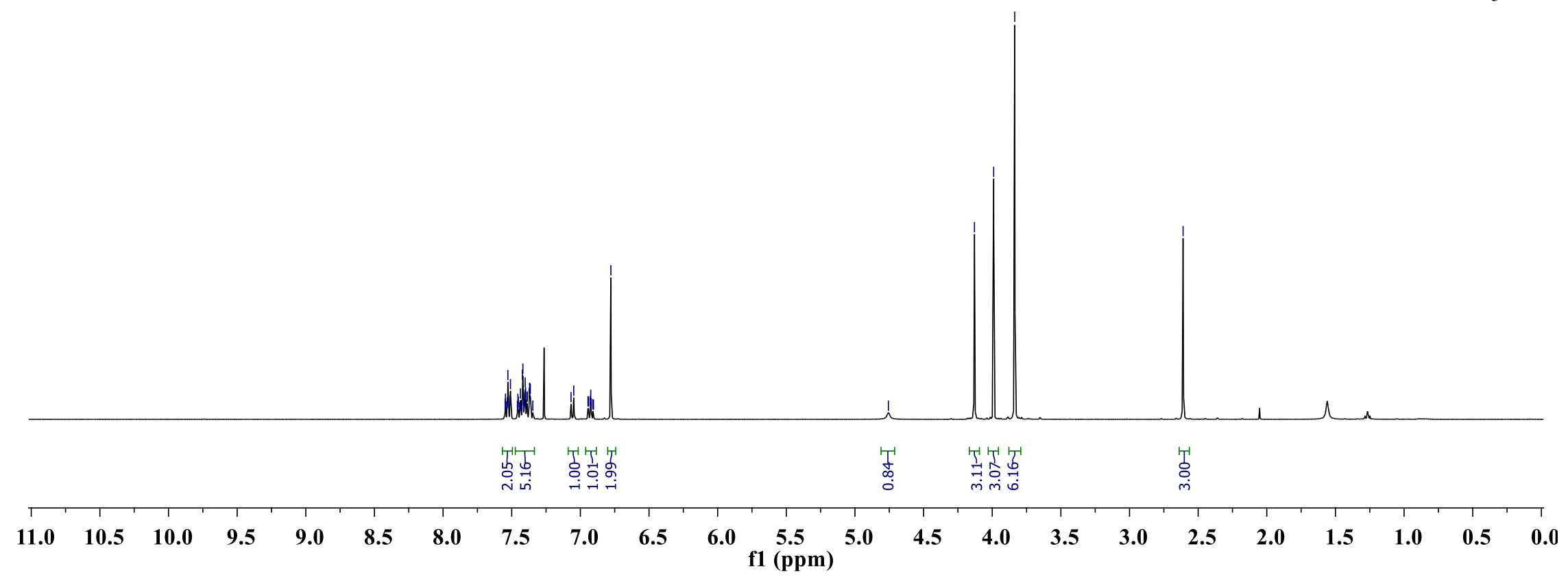




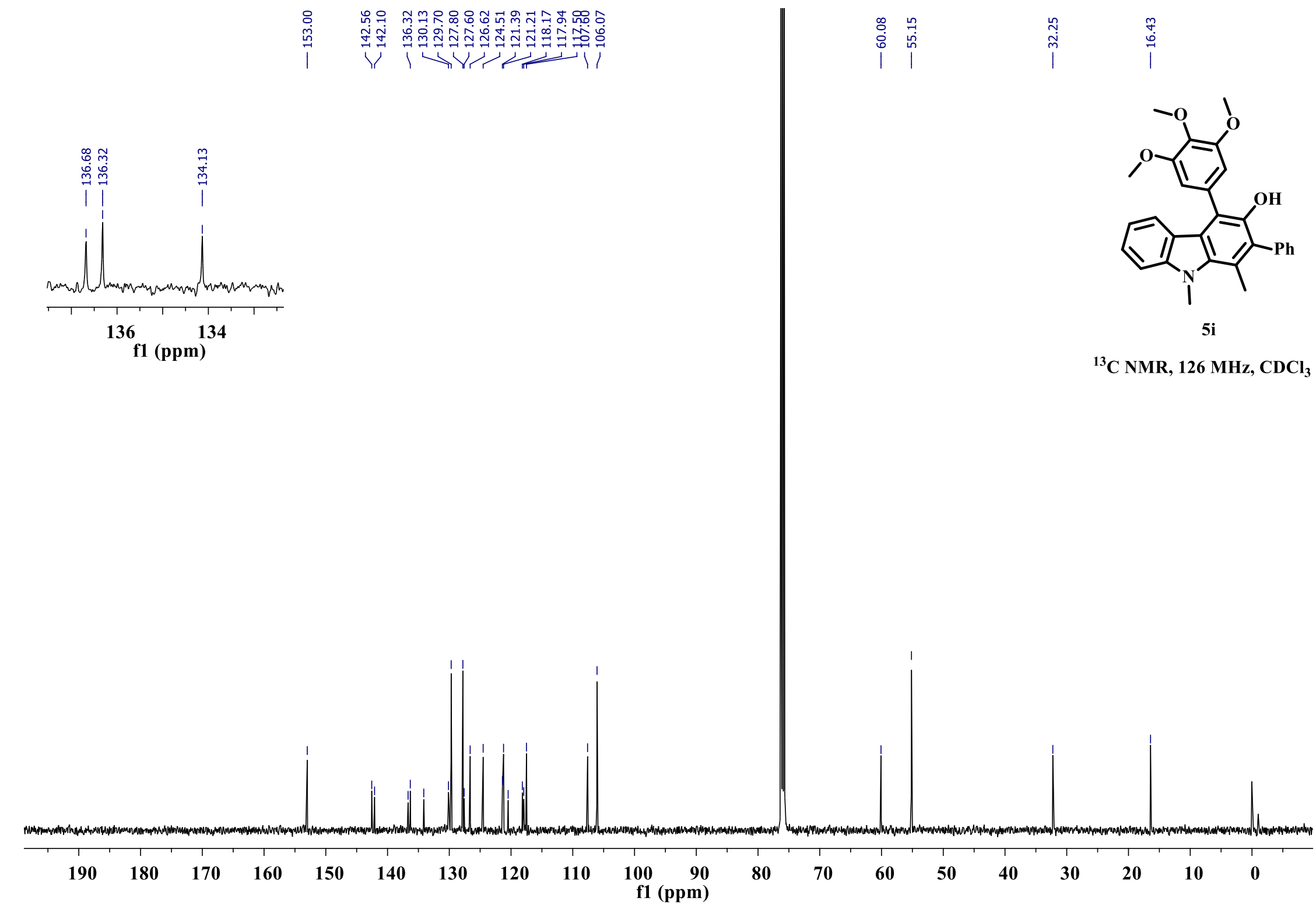




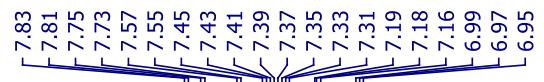

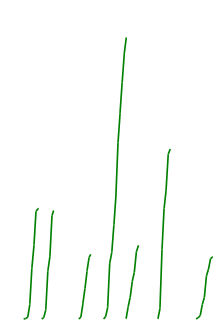

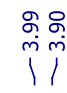

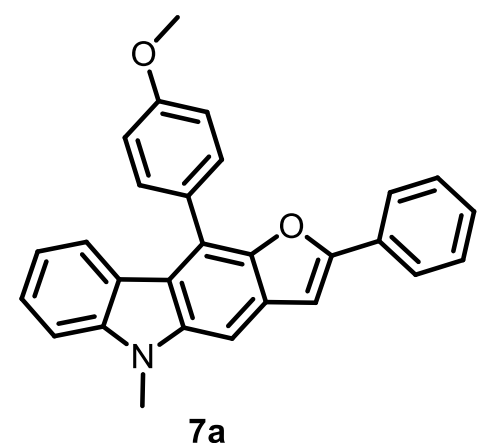

${ }^{1} \mathrm{H} \mathrm{NMR}, 400 \mathrm{MHz}, \mathrm{CDCl}_{3}$

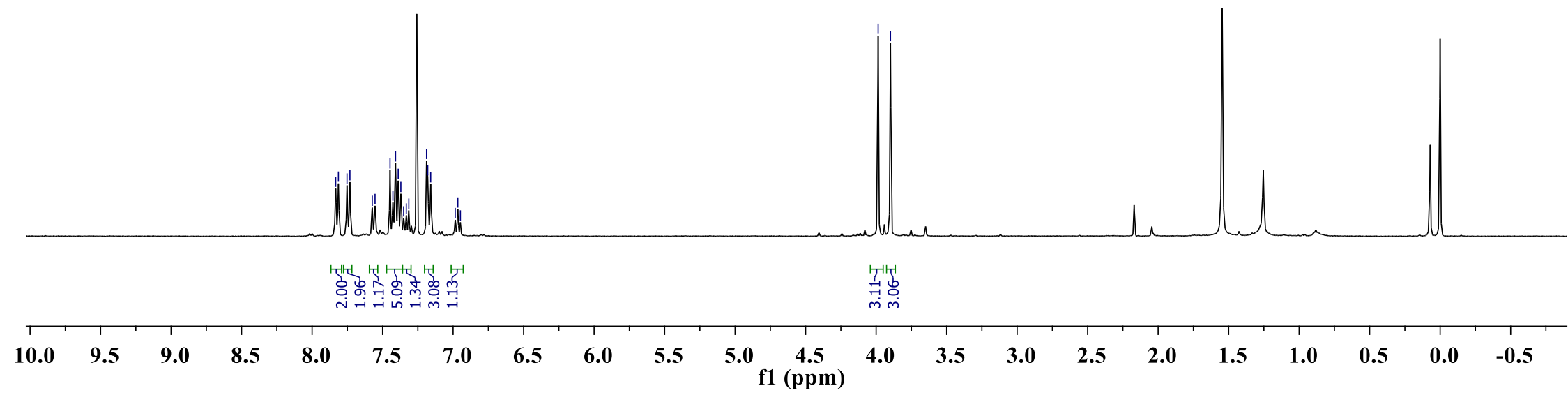




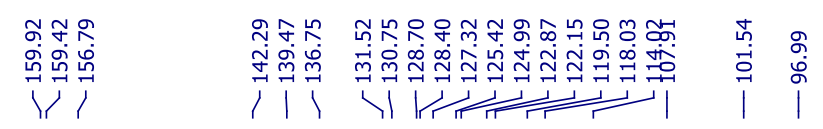

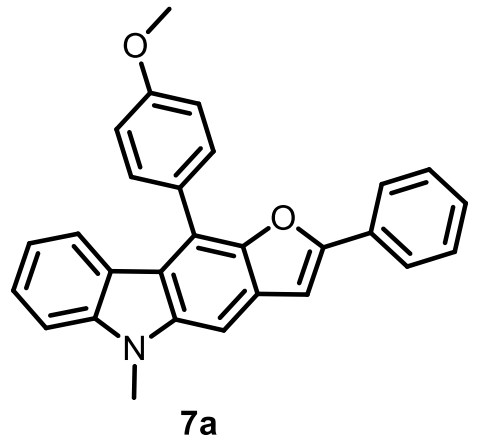

${ }^{13} \mathrm{C}$ NMR, $101 \mathrm{MHz}, \mathrm{CDCl}_{3}$

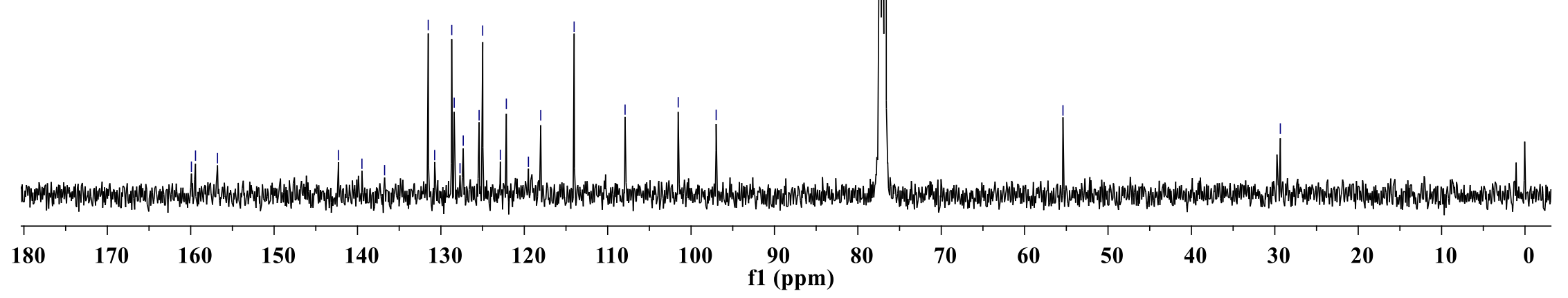




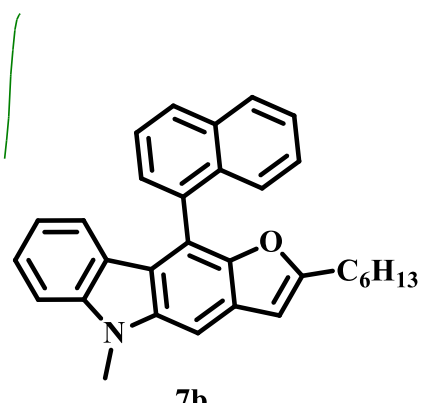

${ }^{1} \mathrm{H}$ NMR, $300 \mathrm{MHz}, \mathrm{CDCl}_{3}$

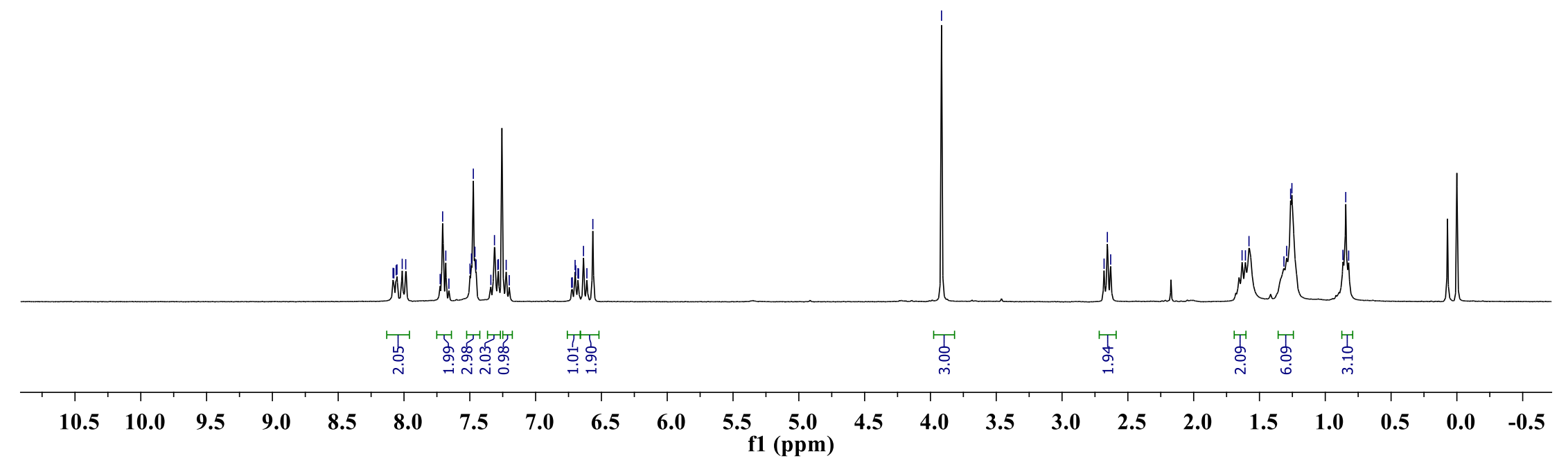




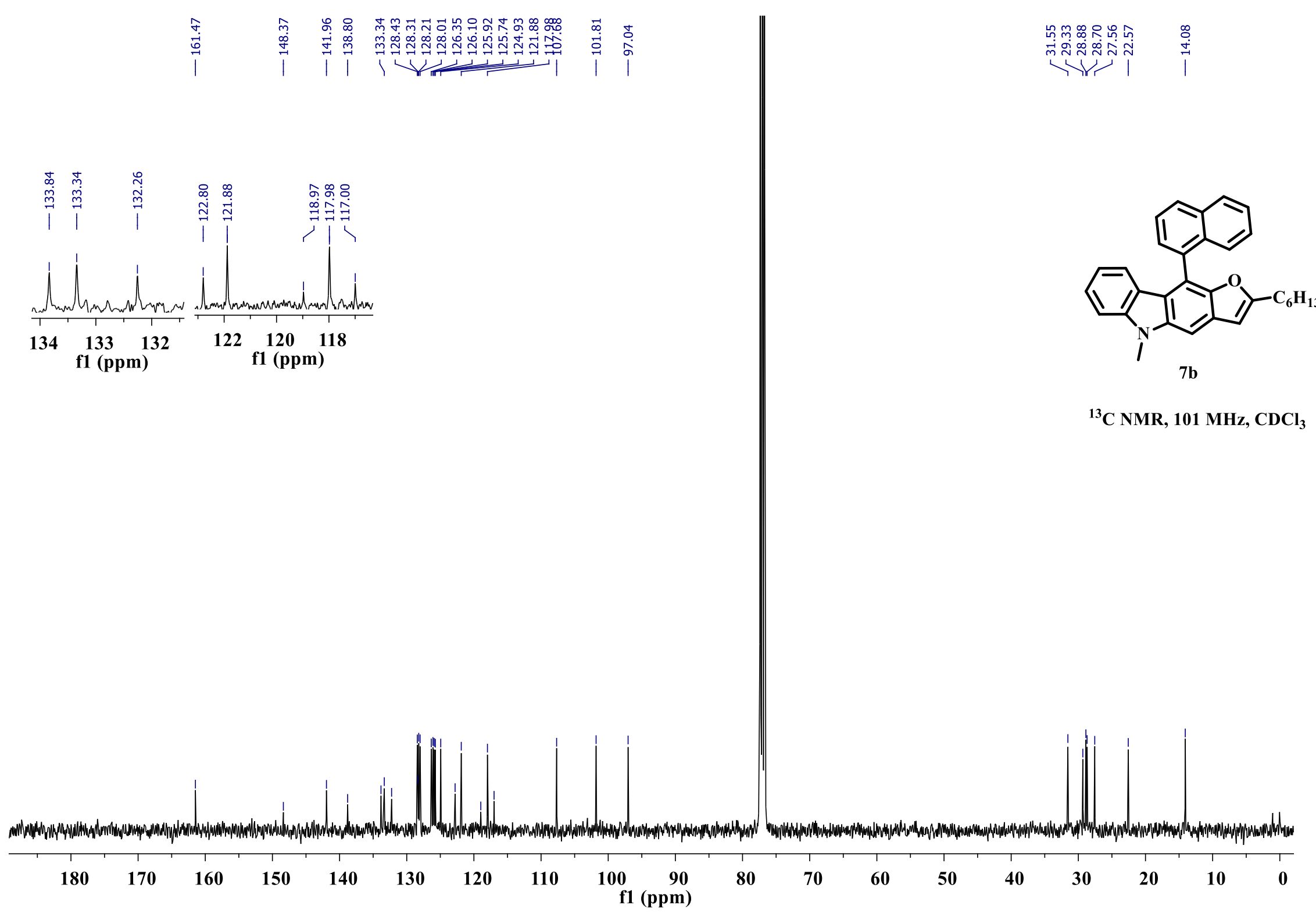


7. HRMS spectral copy of $\mathrm{H}_{2} \mathrm{O}^{18}$ experiment

CRR_3919_53_351

07NOV2019_46 52 (0.497) AM2 (Ar,22000.0,0.00,0.00); ABS; Cm (49:52)

1007
352.1583

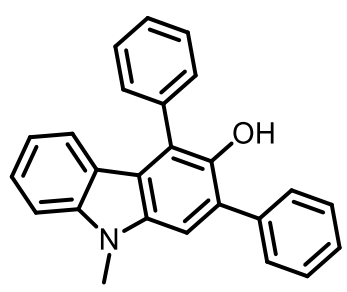

HRMS- ${ }^{18} \mathrm{O}-3 \mathrm{a}$ and $3 \mathrm{a}$
1: TOF MS ES+

$2.43 \mathrm{e} 7$

S97 


\section{Elemental Composition Report}

Page 1

\section{Single Mass Analysis}

Tolerance $=2.0$ PPM / DBE: $\min =-1.5, \max =50.0$

Element prediction: Off

Number of isotope peaks used for i-FIT $=3$

Monoisotopic Mass, Even Electron lons

19 formula(e) evaluated with 1 results within limits (up to 50 closest results for each mass)

Elements Used:

C: $0-25 \quad H: 0-20 \quad \mathrm{~N}: 0-2 \quad$ 180: 0-4

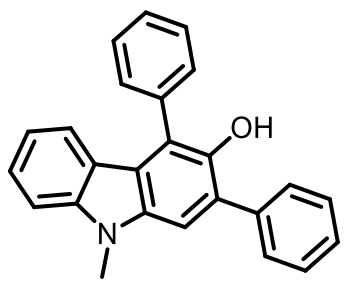

CRR_3919_53_351

07NŌV2019 $44 \overline{6} 53$ (0.506) AM2 (Ar,22000.0,0.00,0.00); ABS; Cm (48:54)

HRMS- ${ }^{18} \mathrm{O}-3 \mathrm{a}$ and $3 \mathrm{a}$

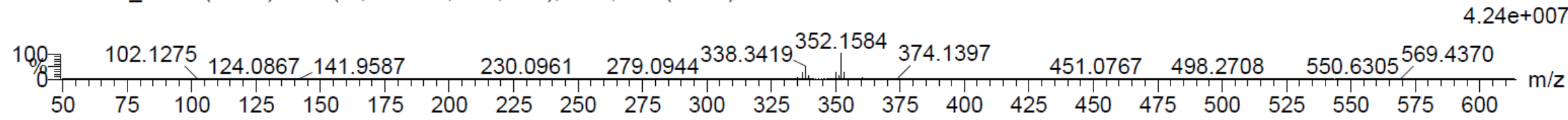

Minimum:

Maximum:

$\begin{array}{lll}5.0 & 2.0 \quad & -1.5 \\ & & 50.0\end{array}$

Mass

Calc. Mass mDa PPM DBE

i-FIT

Norm

Conf (\%) Formula

$352.1584 \quad 352.1587$

$\begin{array}{llll}-0.3 & -0.9 & 16.5 & 887.2\end{array}$

$\mathrm{n} / \mathrm{a} \quad \mathrm{n} / \mathrm{a}$

C25 H20 N 180 